\title{
LIMIT THEOREMS FOR MARKOV WALKS CONDITIONED TO STAY POSITIVE UNDER A SPECTRAL GAP ASSUMPTION
}

\author{
By Ion Grama, Ronan Lauvergnat and Émile Le Page \\ Université de Bretagne Sud
}

Consider a Markov chain $\left(X_{n}\right)_{n \geq 0}$ with values in the state space $\mathbb{X}$. Let $f$ be a real function on $\mathbb{X}$ and set $S_{n}=\sum_{i=1}^{n} f\left(X_{i}\right), n \geq 1$. Let $\mathbb{P}_{x}$ be the probability measure generated by the Markov chain starting at $X_{0}=x$. For a starting point $y \in \mathbb{R}$, denote by $\tau_{y}$ the first moment when the Markov walk $\left(y+S_{n}\right)_{n \geq 1}$ becomes nonpositive. Under the condition that $S_{n}$ has zero drift, we find the asymptotics of the probability $\mathbb{P}_{x}\left(\tau_{y}>n\right)$ and of the conditional law $\mathbb{P}_{x}\left(y+S_{n} \leq \cdot \sqrt{n} \mid \tau_{y}>n\right)$ as $n \rightarrow+\infty$.

\section{CONTENTS}

1. Introduction . . . . . . . . . . . . . . . . . . . . . . . . . . . . . 1808

2. Main results . . . . . . . . . . . . . . . . . . . . . . . . . . . . 1811

3. Applications . . . . . . . . . . . . . . . . . . . . . . . . . . . . . . 1819

3.1. Affine random walk in $\mathbb{R}^{d}$ conditioned to stay in a half-space . . . . . . . . . . . 1819

3.2. Two components Markov chains in compact sets under the doeblin-fortet condition . 1820

3.3. Markov chains in compact sets under spectral gap assumptions . . . . . . . . . . . 1821

4. Preliminary statements . . . . . . . . . . . . . . . . . . . . . . 1822

4.1. Results for the Brownian motion . . . . . . . . . . . . . . . . . . . . . 1822

4.2. Strong approximation . . . . . . . . . . . . . . . . . . . . . . . 1823

5. Martingale approximation and related assertions . . . . . . . . . . . . . . . . 1824

6. Integrability of the killed martingale and of the killed Markov walk . . . . . . . . . . . 1829

7. Existence and properties of the harmonic function . . . . . . . . . . . . . . 1838

8. Positivity of the harmonic function . . . . . . . . . . . . . . . . . . . . . 1842

9. Asymptotic behaviour of the exit time . . . . . . . . . . . . . . . . . . 1852

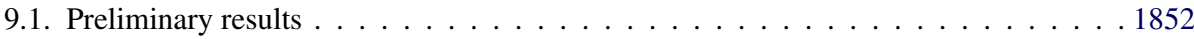

9.2. Proof of Theorem $2.3 \ldots \ldots$. . . . . . . . . . . . . . . . . 1860

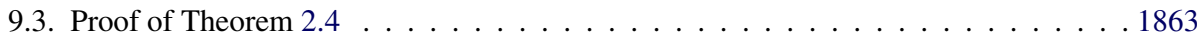

10. Asymptotic behaviour of the conditioned Markov walk . . . . . . . . . . . . 1865

Appendix A: Proofs for affine random walks in $\mathbb{R}^{d} \ldots \ldots \ldots$. . . . . . . . . . . . . . . . . . . . . . . . . .

Appendix B: Proofs for compact Markov chains . . . . . . . . . . . . . . . . . . . . . . . . 1874

Acknowledgements . . . . . . . . . . . . . . . . . . . . . . . . . . 1876

References . . . . . . . . . . . . . . . . . . . . . . . . . 1876

Received July 2016; revised February 2017.

MSC2010 subject classifications. Primary 60G50，60F05，60J50; secondary 60J05，60J70, 60G40.

Key words and phrases. Markov chain, random walk, exit time, harmonic function, limit theorem. 
1. Introduction. Assume that on the probability space $(\Omega, \mathscr{F}, \mathbb{P})$ we are given a sequence of random variables $\left(X_{n}\right)_{n \geq 1}$ with values in a measurable space $\mathbb{X}$. Let $f$ be a real function on $\mathbb{X}$. Suppose that the random walk $S_{n}=$ $\sum_{i=1}^{n} f\left(X_{i}\right), n \geq 1$ has zero drift. For a starting point $y \in \mathbb{R}$, denote by $\tau_{y}$ the time at which $\left(y+S_{n}\right)_{n \geq 1}$ first passes into the interval $(-\infty, 0]$. We are interested in the asymptotic behaviour of the probability $\mathbb{P}\left(\tau_{y}>n\right)$ and of the conditional law of $\frac{y+S_{n}}{\sqrt{n}}$ given the event $\left\{\tau_{y}>n\right\}=\left\{S_{1}>0, \ldots, S_{n}>0\right\}$ as $n \rightarrow+\infty$.

The case when $f$ is the identity function and $\left(X_{n}\right)_{n \geq 1}$ are i.i.d. in $\mathbb{X}=\mathbb{R}$ has been extensively studied in the literature. We refer to Spitzer [31], Iglehart [23, 24], Bolthausen [2], Doney [12], Bertoin and Doney [1], Borovkov [3, 4], Caravenna [6], Vatutin and Wachtel [35] to cite only a few. Recent progress has been made for random walks with independent increments in $\mathbb{X}=\mathbb{R}^{d}$; see Eichelbacher and König [14], Denisov and Wachtel [9, 11] and Duraj [13]. However, to the best of our knowledge, the case of the Markov chains has been treated only in some special cases. Upper and lower bounds for $\mathbb{P}\left(\tau_{y}>n\right)$ have been obtained in Varapoulos [32, 33] for Markov chains with bounded jumps and in Dembo, Ding and Gao [7] for integrated random walks based on independent increments. An approximation of $\mathbb{P}\left(\tau_{y}>n\right)$ by the survival probability of the Brownian motion for Markov walk under moment conditions is given in Varopoulos [34]. Exact asymptotic behaviour was determined in Presman $[29,30]$ in the case of sums of random variables defined on a finite Markov chain under the additional assumption that the distributions have an absolute continuous component and in Denisov and Wachtel [10] for integrated random walks. The case of products of i.i.d. random matrices which reduces to the study of a particular Markov chain defined on a merely compact state space was considered in [20] and the case of affine walks in $\mathbb{R}$ has been treated in [18]. We also point out the work of Denisov, Korshunov and Wachtel [8] where a constructive analysis of harmonic functions for Markov chains with values in $\mathbb{N}$ is performed.

In this paper, we determine the limit of the probability of the exit time $\tau_{y}$ and of the law of $y+S_{n}$ conditioned to stay positive for a Markov chain under the assumption that its transition operator has a spectral gap. In particular, our results cover the case of Markov chains with compact state spaces and the affine random walks in $\mathbb{R}$ (see [18]) and $\mathbb{R}^{d}$ (see Gao, Guivarc'h and Le Page [16]). Our results apply also to the case of sums of i.i.d. random variables.

To present briefly the main results of the paper, denote by $\mathbb{P}_{x}$ and $\mathbb{E}_{x}$ the probability and the corresponding expectation generated by the trajectories of a Markov chain $\left(X_{n}\right)_{n \geq 1}$ with the initial state $X_{0}=x \in \mathbb{X}$. Let $\mathbf{Q}$ be the transition operator of the Markov chain $\left(X_{n}, y+S_{n}\right)_{n \geq 1}$ and let $\mathbf{Q}_{+}$be the restriction of $\mathbf{Q}$ on $\mathbb{X} \times \mathbb{R}_{+}^{*}$. We show that under appropriate assumptions, there exists a $\mathbf{Q}_{+}$-harmonic function $V$ with nonempty support $\operatorname{supp}(V)$ in $\mathbb{X} \times \mathbb{R}$ such that, for any $(x, y) \in \operatorname{supp}(V)$,

$$
\mathbb{P}_{x}\left(\tau_{y}>n\right) \underset{n \rightarrow+\infty}{\sim} \frac{2 V(x, y)}{\sqrt{2 \pi n} \sigma}
$$


and

$$
\mathbb{P}_{x}\left(\frac{y+S_{n}}{\sigma \sqrt{n}} \leq t \mid \tau_{y}>n\right) \underset{n \rightarrow+\infty}{\longrightarrow} \boldsymbol{\Phi}^{+}(t),
$$

where $\boldsymbol{\Phi}^{+}(t)=1-\mathrm{e}^{-\frac{t^{2}}{2}}$ is the Rayleigh distribution function and $\sigma$ is a positive real. Moreover, we complete this result by giving the behaviour of $\mathbb{P}_{x}\left(\tau_{y}>n\right)$ on the complement of $\operatorname{supp}(V)$ : for any $(x, y) \notin \operatorname{supp}(V)$,

$$
\mathbb{P}_{x}\left(\tau_{y}>n\right) \leq c_{x} \mathrm{e}^{-c n}
$$

where $c_{x}$ depends on $x$ and $c$ is a constant. This is different from the case of sums of i.i.d. real random variables, where instead of (1.2), on $\operatorname{supp}(V)^{c}$ it holds $\mathbb{P}_{x}\left(\tau_{y}>n\right)=0$. We give an example of a Markov chain for which the bound (1.2) is attained and state uniform versions of (1.1) and (1.2). A characterization of the $\operatorname{supp}(V)$ is given in point 4 of Theorem 2.2. For details, we refer to Section 2.

The study of the asymptotic behaviour of the probability $\mathbb{P}\left(\tau_{y}>n\right)$ for walks on the real line $\mathbb{R}$ is usually based on the Wiener-Hopf factorization (see Feller [15]). Unfortunately, the Wiener-Hopf factorisation is not well suited for more general walks, as for example, those with values in $\mathbb{R}^{d}$ or for walks with dependent increments. For random walks with dependent increments and for random walks with independent increments in $\mathbb{R}^{d}$, Varopoulos [34], Eichelbacher and König [14] and Denisov and Wachtel [11] have developed an alternative approach based on the existence of the harmonic function. Using the particular structure of the underlaying models such extensions where performed in Denisov and Wachtel [10] for integrated random walks, in [20] for products of random matrices and in [18] for affine random walks in $\mathbb{R}$. Despite these advances, there are still some major difficulties in transferring the harmonic function approach to the case of more general Markov chains. In this paper, we extend it to Markov chains under spectral gap assumptions. Let us highlight below the key points of the proofs.

We begin with the construction of a martingale approximation $\left(M_{n}\right)_{n \geq 1}$ for $\left(S_{n}\right)_{n \geq 1}$ following the approach of Gordin [17]. One of the delicate points of the proof is to control the difference $S_{n}-M_{n}$. We make use of the spectral gap property of the transition operator $\mathbf{P}$ of the Markov chain $\left(X_{n}\right)_{n \geq 1}$ relatively to some Banach space $\mathscr{B}$ (for details we refer to Section 2). Our martingale approximation is such that

$$
\left(z+M_{n}\right)-\left(y+S_{n}\right)=r\left(X_{n}\right),
$$

where $r(x)=\Theta(x)-f(x)$ is the coboundary, $z=y+r(x)$ and $\Theta$ is the solution of the Poisson equation $\Theta-\mathbf{P} \Theta=f$. Under Hypothesis M4, we can control $|r(x)|$ by $c(1+N(x))$ where $N \in \mathscr{B}$ has bounded moments $\mathbb{E}_{x}^{1 / \alpha}\left(N\left(X_{n}\right)^{\alpha}\right) \leq c(1+N(x))$, for some $\alpha>2$. Note that in the case of products of random matrices [20] the coboundary is bounded, so that $\sup _{n \geq 1}\left|S_{n}-M_{n}\right|$ is bounded by a constant $\mathbb{P}_{x}$-a.s. 
for any $x \in \mathbb{X}$, which simplifies greatly the proofs. The extension to the case of unbounded coboundary turns out to be quite laborious even for particular examples. We refer to the case of affine Markov walks considered in [18], where the authors have benefited from the special structure of the model.

The next step is the proof of the existence of a positive harmonic function. The starting idea is very simple. Let $V_{n}(x, y):=\mathbb{E}_{x}\left(\left(y+S_{n}\right) \mathbb{1}_{\left\{\tau_{y}>n\right\}}\right)$ be the expectation of the Markov walk $\left(y+S_{n}\right)_{n \geq 1}$ killed at $\tau_{y}$. Since by the Markov property, we have $V_{n+1}(x, y)=\mathbf{Q}_{+} V_{n}(x, y)$, taking the limit as $n \rightarrow+\infty$ under appropriate assumptions, yields that the function $V(x, y)=\lim _{n \rightarrow+\infty} V_{n}(x, y)$ is $\mathbf{Q}_{+}$harmonic. Using the approximating martingale, the function $V$ can be identified as $V(x, y)=-\mathbb{E}_{x}\left(M_{\tau_{y}}\right)$. To justify this approach, it is important to control uniformly in $n$ the expectation $w_{n}:=\mathbb{E}_{x}\left(\left(z+M_{n}\right) \mathbb{1}_{\left\{\tau_{y}>n\right\}}\right)$. Our key idea (in contrast to [20] and [18]) is the introduction of two extra stopping times $T_{z}$ and $\hat{T}_{z}$ : the first time when $\left(z+M_{n}\right)_{n \geq 1}$ leaves $\mathbb{R}_{+}^{*}$ and the first time larger than $\tau_{y}$ when $\left(z+M_{n}\right)_{n \geq 1}$ leaves $\mathbb{R}_{+}^{*}$, respectively, where as before $z=y+r(x)$. Clearly, $\hat{T}_{z}$ depends on $\tau_{y}$ and dominates both, $\tau_{y}$ and $T_{z}$. The relation of the time $\hat{T}_{z}$ to the exit times $\tau_{y}$ and $T_{z}$ is explicitly given in Lemma 5.3 which is an application of the Markov property to $\hat{T}_{z}$. This property is useful to control uniformly in $n$ the expectation $u_{n}:=\mathbb{E}_{x}\left(\left(z+M_{n}\right) \mathbb{1}_{\left\{\hat{T}_{z}>n\right\}}\right)$, which is one of the crucial points of the proof. To establish this we note that the sequence $\left(u_{n}\right)_{n \geq 0}$ is increasing, since $\left(\left(z+M_{n}\right) \mathbb{1}_{\left\{\hat{T}_{z}>n\right\}}\right)_{n \geq 1}$ is a submartingale. In addition, we show that it satisfies a recurrence equation, which implies its boundedness. Using the previous arguments, we obtain a uniform control on the expectation $w_{n}$. All the details can be found in Sections 6 and 7. The proof of the (strict) positivity of $V$ is also rather involved but uses similar arguments based on the subhamonicity of the function $\hat{W}(x, z)=-\mathbb{E}_{x}\left(M_{\hat{T}_{z}}\right)$. (See Section 8.)

Now we can turn to the tail behaviour of the exit time $\tau_{y}$. It is inferred from that of the exit time $\tau_{y}^{b m}$ of the Brownian motion, using the Donsker invariance principle for sums defined on Markov chains with a the rate of convergence, recently proved in [19]. The result in [19] gives the explicit dependence of the constants on the norm $\left\|\boldsymbol{\delta}_{x}\right\|_{\mathscr{B}^{\prime}}$ of the Dirac measure $\boldsymbol{\delta}_{x}$ and on the absolute moments $\mu_{\alpha}(x)=\sup _{n \geq 1} \mathbb{E}_{x}^{1 / \alpha}\left(\left|f\left(X_{n}\right)\right|^{\alpha}\right)$ for some initial state $x \in \mathbb{X}$ and some $\alpha>2$. To have a control on the constants we make use of Hypothesis M4. Note that for products of random matrices [20], $\left\|\boldsymbol{\delta}_{x}\right\|_{\mathscr{B}^{\prime}}$ and $\mu_{\alpha}(x)$ are bounded uniformly in the initial state $x \in \mathbb{X}$, so that the rate of convergence invariance principle does not depend on the initial state. The case of when $\left\|\boldsymbol{\delta}_{x}\right\|_{\mathscr{B}^{\prime}}$ and $\mu_{\alpha}(x)$ are not bounded was studied in details in [18] for affine Markov walks.

The paper is organized as follows. In Section 2, we introduce the necessary notations and state our main results. In Section 3, we give applications of the results of the paper to stochastic recursions in $\mathbb{R}^{d}$ and Markov chains with compact state space. In Section 4, we collect some preliminary results. In Section 5, we construct 
the approximating martingale and state some of its properties and of the associated exit times. In Section 6, we prove that the expectations $\mathbb{E}_{x}\left(\left(y+S_{n}\right) \mathbb{1}_{\left\{\tau_{y}>n\right\}}\right)$ are bounded uniformly in $n$. Using the results of Sections 5 and 6, we establish in Section 7 the existence of a $\mathbf{Q}_{+}$-harmonic function and prove in Section 8 that this function is not identically zero. We determine the limit of the probability $\mathbb{P}_{x}\left(\tau_{y}>n\right)$ in Section 9 and that of the conditioned law of $\left(y+S_{n}\right) /(\sigma \sqrt{n})$ given the event $\left\{\tau_{y}>n\right\}$ in Section 10 .

We end this section by setting some basic notations. For the rest of the paper, the symbol $c$ denotes a positive constant depending on the all previously introduced constants. Sometimes, to stress the dependence of the constants on some parameters $\alpha, \beta, \ldots$ we shall use the notations $c_{\alpha}, c_{\alpha, \beta}, \ldots$ All these constants are likely to change their values every occurrence. For any real numbers $u$ and $v$, denote by $u \wedge v=\min (u, v)$ the minimum between $u$ and $v$. The indicator of an event $A$ is denoted by $\mathbb{1}_{A}$. For any bounded measurable function $f$ on $\mathbb{X}$, random variable $X$ in $\mathbb{X}$ and event $A$, the integral $\int_{\mathbb{X}} f(x) \mathbb{P}(X \in \mathrm{d} x, A)$ means the expectation $\mathbb{E}(f(X) ; A)=\mathbb{E}\left(f(X) \mathbb{1}_{A}\right)$.

2. Main results. Let $\left(X_{n}\right)_{n \geq 0}$ be a Markov chain taking values in the measurable state space $(\mathbb{X}, \mathscr{X})$, defined on the probability space $(\Omega, \mathscr{F}, \mathbb{P})$. For any given $x \in \mathbb{X}$, denote by $\mathbf{P}(x, \cdot)$ its transition probability, to which we associate the transition operator

$$
\mathbf{P} g(x)=\int_{\mathbb{X}} g\left(x^{\prime}\right) \mathbf{P}\left(x, \mathrm{~d} x^{\prime}\right),
$$

for any complex bounded measurable function $g$ on $\mathbb{X}$. Denote by $\mathbb{P}_{x}$ and $\mathbb{E}_{x}$ the probability and the corresponding expectation generated by the finite dimensional distributions of the Markov chain $\left(X_{n}\right)_{n \geq 0}$ starting at $X_{0}=x$. We remark that $\mathbf{P} g(x)=\mathbb{E}_{x}\left(g\left(X_{1}\right)\right)$ and $\mathbf{P}^{n} g(x)=\mathbb{E}_{x}\left(g\left(X_{n}\right)\right)$ for any $g$ complex bounded measurable, $x \in \mathbb{X}$ and $n \geq 1$.

Let $f$ be a real valued function defined on the state space $\mathbb{X}$ and let $\mathscr{B}$ be a Banach space of complex valued functions on $\mathbb{X}$ endowed with the norm $\|\cdot\|_{\mathscr{B}}$. Let $\|\cdot\|_{\mathscr{B} \rightarrow \mathscr{B}}$ be the operator norm on $\mathscr{B}$ and let $\mathscr{B}^{\prime}=\mathscr{L}(\mathscr{B}, \mathbb{C})$ be the topological dual of $\mathscr{B}$ endowed with the norm $\|\varphi\|_{\mathscr{B}}=\sup _{h \in \mathscr{B}} \frac{|\varphi(h)|}{\|h\|_{\mathscr{B}}}$, for any $\varphi \in \mathscr{B}^{\prime}$. Denote by $e$ the unit function of $\mathbb{X}: e(x)=1$, for any $x \in \mathbb{X}$ and by $\delta_{x}$ the Dirac measure at $x \in \mathbb{X}: \boldsymbol{\delta}_{x}(g)=g(x)$, for any $g \in \mathscr{B}$.

Following [19], we assume the following hypotheses.

Hypothesis M1 (Banach space). 1. The unit function e belongs to $\mathscr{B}$.

2. For any $x \in \mathbb{X}$, the Dirac measure $\boldsymbol{\delta}_{x}$ belongs to $\mathscr{B}^{\prime}$.

3. The Banach space $\mathscr{B}$ is included in $L^{1}(\mathbf{P}(x, \cdot))$, for any $x \in \mathbb{X}$.

4. There exists a constant $\kappa \in(0,1)$ such that for any $g \in \mathscr{B}$, the function $\mathrm{e}^{\text {itf }} g$ is in $\mathscr{B}$ for any $t$ satisfying $|t| \leq \kappa$.

Under point 3 of $\mathrm{M} 1, \mathbf{P} g(x)$ exists for any $g \in \mathscr{B}$ and $x \in \mathbb{X}$. 
HYPOTHESIS M2 (Spectral gap). 1. The map $g \mapsto \mathbf{P} g$ is a bounded operator on $\mathscr{B}$.

2. There exist constants $c_{1}>0$ and $c_{2}>0$ such that

$$
\mathbf{P}=\Pi+Q,
$$

where $\Pi$ is a one-dimensional projector and $Q$ is an operator on $\mathscr{B}$ satisfying $\Pi Q=Q \Pi=0$ and for any $n \geq 1$,

$$
\left\|Q^{n}\right\|_{\mathscr{B} \rightarrow \mathscr{B}} \leq c_{1} \mathrm{e}^{-c_{2} n} .
$$

Since $\Pi$ is a one-dimensional projector and $e$ is an eigenvector of $\mathbf{P}$, there exists a linear form $\boldsymbol{v} \in \mathscr{B}^{\prime}$, such that for any $g \in \mathscr{B}$,

$$
\Pi g=\boldsymbol{v}(g) e .
$$

When Hypotheses M1 and M2 hold, we set $\mathbf{P}_{t} g:=\mathbf{P}\left(\mathrm{e}^{i t f} g\right)$ for any $g \in \mathscr{B}$ and $t \in[-\kappa, \kappa]$. In particular, $\mathbf{P}_{0}=\mathbf{P}$.

HYPOTHESIS M3 (Perturbed transition operator). 1. For any $|t| \leq \kappa$, the map $g \mapsto \mathbf{P}_{t} g$ is a bounded operator on $\mathscr{B}$.

2. There exists a constant $C_{\mathbf{P}}>0$ such that, for any $n \geq 1$ and $|t| \leq \kappa$,

$$
\left\|\mathbf{P}_{t}^{n}\right\|_{\mathscr{B} \rightarrow \mathscr{B}} \leq C_{\mathbf{P}} .
$$

The following hypothesis will be important for establishing the main results.

Hypothesis M4 (Local integrability). The Banach space $\mathscr{B}$ contains a sequence of real nonnegative functions $N, N_{1}, N_{2}, \ldots$ such that:

1. There exist $\alpha>2$ and $\gamma>0$ such that, for any $x \in \mathbb{X}$,

$$
\max \left\{|f(x)|^{1+\gamma},\left\|\boldsymbol{\delta}_{x}\right\|_{\mathscr{B}^{\prime}}, \mathbb{E}_{x}^{1 / \alpha}\left(N\left(X_{n}\right)^{\alpha}\right)\right\} \leq c(1+N(x))
$$

and

$$
N(x) \mathbb{1}_{\{N(x)>l\}} \leq N_{l}(x), \quad \text { for any } l \geq 1 .
$$

2. There exists $c>0$ such that, for any $l \geq 1$,

$$
\left\|N_{l}\right\|_{\mathscr{B}} \leq c .
$$

3. There exist $\beta>0$ and $c>0$ such that, for any $l \geq 1$,

$$
\left|\boldsymbol{v}\left(N_{l}\right)\right| \leq \frac{c}{l^{1+\beta}} .
$$


A comment on Hypothesis M4 seems to be appropriate. Although the function $N$ belongs to the Banach space $\mathscr{B}$, the truncated function $x \mapsto N(x) \mathbb{1}_{\{N(x)>l\}}$ may not belong to $\mathscr{B}$. Fortunately, in many interesting cases, there exists an element $N_{l}$ in $\mathscr{B}$ dominating it. We refer to Section 3, where we verify Hypothesis M4 for stochastic recursions in $\mathbb{R}^{d}$ and for Markov chains with compact state space. Note also that the function $f$ need not belong to the Banach space $\mathscr{B}$.

Under Hypotheses M1, M2 and M4, we have, for any $x \in \mathbb{X}$ and $n \geq 1$,

$$
\begin{aligned}
\mathbb{E}_{x}\left(N\left(X_{n}\right)\right) & =\boldsymbol{v}(N)+Q^{n} N(x) \\
& \leq|\boldsymbol{v}(N)|+\left\|Q^{n}\right\|_{\mathscr{B} \rightarrow \mathscr{B}}\|N\|_{\mathscr{B}}\left\|\boldsymbol{\delta}_{x}\right\|_{\mathscr{B}^{\prime}} \\
& \leq c\left(1+\mathrm{e}^{-c n} N(x)\right)
\end{aligned}
$$

and, in the same way, for any $x \in \mathbb{X}, l \geq 1$ and $n \geq 1$,

$$
\mathbb{E}_{x}\left(N_{l}\left(X_{n}\right)\right) \leq \frac{c}{l^{1+\beta}}+c \mathrm{e}^{-c n}(1+N(x))
$$

Moreover, from point 1 of 44 , one can easily verify that, for any $x \in \mathbb{X}$,

$$
\mu_{\alpha}(x):=\sup _{n \geq 1} \mathbb{E}_{x}^{1 / \alpha}\left(\left|f\left(X_{n}\right)\right|^{\alpha}\right) \leq c\left(1+N(x)^{\frac{1}{1+\gamma}}\right) .
$$

The following proposition is proved in [19], where the bounds on the right follow from (2.4) and again M4.

Proposition 2.1. Assume that the Markov chain $\left(X_{n}\right)_{n \geq 0}$ and the function $f$ satisfy Hypotheses M1-M4.

1. There exists a constant $\mu$ such that, for any $x \in \mathbb{X}$ and $n \geq 1$,

$$
\left|\mathbb{E}_{x}\left(f\left(X_{n}\right)\right)-\mu\right| \leq c \mathrm{e}^{-c n}\left(1+\mu_{\alpha}(x)^{1+\gamma}+\left\|\boldsymbol{\delta}_{x}\right\|_{\mathscr{B}^{\prime}}\right) \leq c \mathrm{e}^{-c n}(1+N(x)) .
$$

2. There exists a constant $\sigma \geq 0$ such that, for any $x \in \mathbb{X}$ and $n \geq 1$,

$$
\begin{aligned}
\sup _{m \geq 0}\left|\operatorname{Var}_{x}\left(\sum_{k=m+1}^{m+n} f\left(X_{k}\right)\right)-n \sigma^{2}\right| & \leq c\left(1+\mu_{\alpha}(x)^{2+2 \gamma}+\left\|\boldsymbol{\delta}_{x}\right\|_{\mathscr{B}^{\prime}}\right) \\
& \leq c\left(1+N(x)^{2}\right),
\end{aligned}
$$

where $\operatorname{Var}_{x}$ is the variance under $\mathbb{P}_{x}$.

We do not assume the existence of the stationary probability measure. If a stationary probability measure $\boldsymbol{v}^{\prime}$ satisfying $\boldsymbol{v}^{\prime}\left(N^{2}\right)<+\infty$ exists then, under Hypotheses M1-M4, we have that $\boldsymbol{v}^{\prime}=\boldsymbol{v}$ is necessarily unique and it holds (see [19])

$$
\boldsymbol{v}(f)=\mu \quad \text { and } \quad \sigma^{2}=\boldsymbol{v}\left(f^{2}\right)-\boldsymbol{v}(f)^{2}+2 \sum_{n=1}^{+\infty}\left[\boldsymbol{v}\left(f P^{n} f\right)-\boldsymbol{v}(f)^{2}\right]
$$


HyPothesis M5 (Centring and nondegeneracy). We suppose that the constants $\mu$ and $\sigma$ defined in Proposition 2.1 satisfy $\mu=0$ and $\sigma>0$.

Under M5 it follows from Proposition 2.1 that, for any $x \in \mathbb{X}$ and $n \geq 1$,

$$
\left|\mathbb{E}_{x}\left(f\left(X_{n}\right)\right)\right| \leq c \mathrm{e}^{-c n}(1+N(x)) .
$$

Let $y \in \mathbb{R}$ be a starting point and $\left(y+S_{n}\right)_{n \geq 0}$ be the Markov walk defined by $S_{n}:=\sum_{k=1}^{n} f\left(X_{k}\right), n \geq 1$ with $S_{0}=0$. Denote by $\tau_{y}$ the first moment when $y+S_{n}$ becomes nonpositive:

$$
\tau_{y}:=\inf \left\{k \geq 1: y+S_{k} \leq 0\right\} .
$$

It is shown in Lemma 5.5 that for any $y \in \mathbb{R}$ and $x \in \mathbb{X}$, the stopping time $\tau_{y}$ is $\mathbb{P}_{x}$-a.s. finite. The asymptotic behaviour of the probability $\mathbb{P}_{x}\left(\tau_{y}>n\right)$ is determined by the harmonic function which we proceed to introduce. For any $(x, y) \in \mathbb{X} \times \mathbb{R}$, denote by $\mathbf{Q}(x, y, \cdot)$ the transition probability of the Markov chain $\left(X_{n}, y+S_{n}\right)_{n \geq 0}$. The restriction of the measure $\mathbf{Q}(x, y, \cdot)$ on $\mathbb{X} \times \mathbb{R}_{+}^{*}$ is defined by

$$
\mathbf{Q}_{+}(x, y, B)=\mathbf{Q}(x, y, B)
$$

for any measurable set $B$ on $\mathbb{X} \times \mathbb{R}_{+}^{*}$ and for any $(x, y) \in \mathbb{X} \times \mathbb{R}$. For any bounded measurable function $\varphi: \mathbb{X} \times \mathbb{R} \rightarrow \mathbb{R}$ set $\mathbf{Q}_{+} \varphi(x, y)=\int_{\mathbb{X} \times \mathbb{R}_{+}^{*}} \varphi\left(x^{\prime}, y^{\prime}\right) \mathbf{Q}_{+}(x, y$, $\left.\mathrm{d} x^{\prime} \times \mathrm{d} y^{\prime}\right)$, where $(x, y) \in \mathbb{X} \times \mathbb{R}$. A function $V: \mathbb{X} \times \mathbb{R} \rightarrow \mathbb{R}$ is said to be $\mathbf{Q}_{+}$-harmonic if

$$
\mathbf{Q}_{+} V(x, y)=V(x, y), \quad \text { for any }(x, y) \in \mathbb{X} \times \mathbb{R} .
$$

We shall deal only with nonnegative harmonic functions $V$. Denote by $\operatorname{supp}(V)$ the support of such a function $V$,

$$
\operatorname{supp}(V):=\{(x, y) \in \mathbb{X} \times \mathbb{R}: V(x, y)>0\} .
$$

On the complement of $\operatorname{supp}(V)$, the function $V$ is 0 . For any $\gamma>0$, consider the set

$$
\mathscr{D}_{\gamma}:=\left\{(x, y) \in \mathbb{X} \times \mathbb{R}: \exists n_{0} \geq 1, \mathbb{P}_{x}\left(y+S_{n_{0}}>\gamma\left(1+N\left(X_{n_{0}}\right)\right), \tau_{y}>n_{0}\right)>0\right\} .
$$

The following assertion proves the existence of a nonidentically zero harmonic function.

THEOREM 2.2. Assume Hypotheses M1-M5.

1. For any $x \in \mathbb{X}, y \in \mathbb{R}$, the sequence $\left(\mathbb{E}_{x}\left(y+S_{n} ; \tau_{y}>n\right)\right)_{n \geq 0}$ converges to a real number $V(x, y)$ :

$$
\mathbb{E}_{x}\left(y+S_{n} ; \tau_{y}>n\right) \underset{n \rightarrow+\infty}{\longrightarrow} V(x, y) .
$$


2. The function $V: \mathbb{X} \times \mathbb{R} \rightarrow \mathbb{R}$, defined in the previous point is $\mathbf{Q}_{+}$-harmonic, that is, for any $x \in \mathbb{X}, y \in \mathbb{R}$,

$$
\mathbf{Q}_{+} V(x, y)=\mathbb{E}_{x}\left(V\left(X_{1}, y+S_{1}\right) ; \tau_{y}>1\right)=V(x, y) .
$$

3. For any $x \in \mathbb{X}$, the function $V(x, \cdot)$ is nonnegative and nondecreasing on $\mathbb{R}$ and

$$
\lim _{y \rightarrow+\infty} \frac{V(x, y)}{y}=1 .
$$

Moreover, for any $\delta>0, x \in \mathbb{X}$ and $y \in \mathbb{R}$,

$$
\begin{aligned}
(1-\delta) \max (y, 0)-c_{\delta}(1+N(x)) & \leq V(x, y) \\
& \leq(1+\delta) \max (y, 0)+c_{\delta}(1+N(x)) .
\end{aligned}
$$

4. There exists $\gamma_{0}>0$ such that, for any $\gamma \geq \gamma_{0}$,

$$
\operatorname{supp}(V)=\mathscr{D}_{\gamma} .
$$

The following result gives the asymptotic of the exit probability for fixed $(x, y) \in \mathbb{X} \times \mathbb{R}$.

THEOREM 2.3. Assume Hypotheses M1-M5.

1. For any $(x, y) \in \operatorname{supp}(V)$,

$$
\mathbb{P}_{x}\left(\tau_{y}>n\right) \underset{n \rightarrow+\infty}{\sim} \frac{2 V(x, y)}{\sqrt{2 \pi n} \sigma} .
$$

2. For any $(x, y) \notin \operatorname{supp}(V)$ and $n \geq 1$,

$$
\mathbb{P}_{x}\left(\tau_{y}>n\right) \leq c \mathrm{e}^{-c n}(1+N(x)) .
$$

Now we complete point 1 of the previous theorem by some estimations.

THEOREM 2.4. Assume Hypotheses M1-M5.

1. There exists $\varepsilon_{0}>0$ such that, for any $\varepsilon \in\left(0, \varepsilon_{0}\right), n \geq 1$ and $(x, y) \in \mathbb{X} \times \mathbb{R}$,

$$
\left|\mathbb{P}_{x}\left(\tau_{y}>n\right)-\frac{2 V(x, y)}{\sqrt{2 \pi n} \sigma}\right| \leq c_{\varepsilon} \frac{\max (y, 0)+\left(1+y \mathbb{1}_{\left\{y>n^{1 / 2-\varepsilon}\right\}}+N(x)\right)^{2}}{n^{1 / 2+\varepsilon / 16}} .
$$

2. Moreover, for any $(x, y) \in \mathbb{X} \times \mathbb{R}$ and $n \geq 1$,

$$
\mathbb{P}_{x}\left(\tau_{y}>n\right) \leq c \frac{1+\max (y, 0)+N(x)}{\sqrt{n}} .
$$

Finally, we give the asymptotic of the conditional law of $y+S_{n}$. 
THEOREM 2.5. Assume Hypotheses M1-M5.

1. For any $(x, y) \in \operatorname{supp}(V)$ and $t \geq 0$,

$$
\mathbb{P}_{x}\left(\frac{y+S_{n}}{\sigma \sqrt{n}} \leq t \mid \tau_{y}>n\right) \underset{n \rightarrow+\infty}{\longrightarrow} \Phi^{+}(t),
$$

where $\boldsymbol{\Phi}^{+}(t)=1-\mathrm{e}^{-\frac{t^{2}}{2}}$ is the Rayleigh distribution function.

2. Moreover, there exists $\varepsilon_{0}>0$ such that, for any $\varepsilon \in\left(0, \varepsilon_{0}\right), n \geq 1, t_{0}>0$, $t \in\left[0, t_{0}\right]$ and $(x, y) \in \mathbb{X} \times \mathbb{R}$,

$$
\begin{aligned}
\left|\mathbb{P}_{x}\left(y+S_{n} \leq t \sqrt{n}, \tau_{y}>n\right)-\frac{2 V(x, y)}{\sqrt{2 \pi n} \sigma} \boldsymbol{\Phi}^{+}\left(\frac{t}{\sigma}\right)\right| & \\
\leq c_{\varepsilon, t_{0}} & \frac{\max (y, 0)+\left(1+y \mathbb{1}_{\left\{y>n^{1 / 2-\varepsilon}\right\}}+N(x)\right)^{2}}{n^{1 / 2+\varepsilon / 16}} .
\end{aligned}
$$

We now comment on Theorems 2.2 and 2.3.

REMARK 2.6. If we assume that there exist $\delta>0$ and $M>0$ such that for any $x \in \mathbb{X}, \mathbb{P}_{x}\left(f\left(X_{1}\right)>\delta, N\left(X_{1}\right) \leq M\right)>0$, then one can see that the set $\mathbb{X} \times[0,+\infty)$ is included in $\operatorname{supp}(V)$.

REMARK 2.7. The sets $\left(\mathscr{D}_{\gamma}\right)_{\gamma>0}$ are nested and become equal to $\operatorname{supp}(V)$ for large $\gamma$ : we have $\mathscr{D}_{\gamma_{1}} \supseteq \mathscr{D}_{\gamma_{2}} \supseteq \mathscr{D}_{\gamma}=\operatorname{supp}(V)$, for $\gamma_{1} \leq \gamma_{2} \leq \gamma$, where $\gamma$ is large enough (see Proposition 8.8).

REMARK 2.8. The set $\operatorname{supp}(V)$ is not empty. More precisely, there exists $\gamma_{1}>$ 0 such that

$$
\left\{(x, y) \in \mathbb{X} \times \mathbb{R}: y>\gamma_{1}(1+N(x))\right\} \subseteq \operatorname{supp}(V) ;
$$

see Proposition 8.8. Example 2.11 and Figure 1 illustrate this property.

REMARK 2.9. When $\left(X_{n}\right)_{n \geq 1}$ are i.i.d., it is well known that $\mathbb{P}_{x}\left(\tau_{y}>n\right)=0$ for any $(x, y) \notin \operatorname{supp}(V)$. When the sequence $\left(X_{n}\right)_{n \geq 1}$ is a Markov chain, instead of this, we have an exponential bound; see point 2 of Theorem 2.3. We show that this bound is attained for some Markov walk. We refer for details to Example 2.12 .

EXAMPLE 2.10 (Random walks in $\mathbb{R}$ ). Suppose that $\left(X_{n}\right)_{n \geq 1}$ are i.i.d. real random variables of mean 0 and positive variance with finite absolute moments of order $p>2$. In this case, one can take $N=N_{l}=0, l \geq 0$. Therefore,

$$
\mathscr{D}_{\gamma}:=\left\{y \in \mathbb{R}: \exists n_{0} \geq 1, \mathbb{P}\left(y+S_{n_{0}}>\gamma, \tau_{y}>n_{0}\right)>0\right\} \text {. }
$$




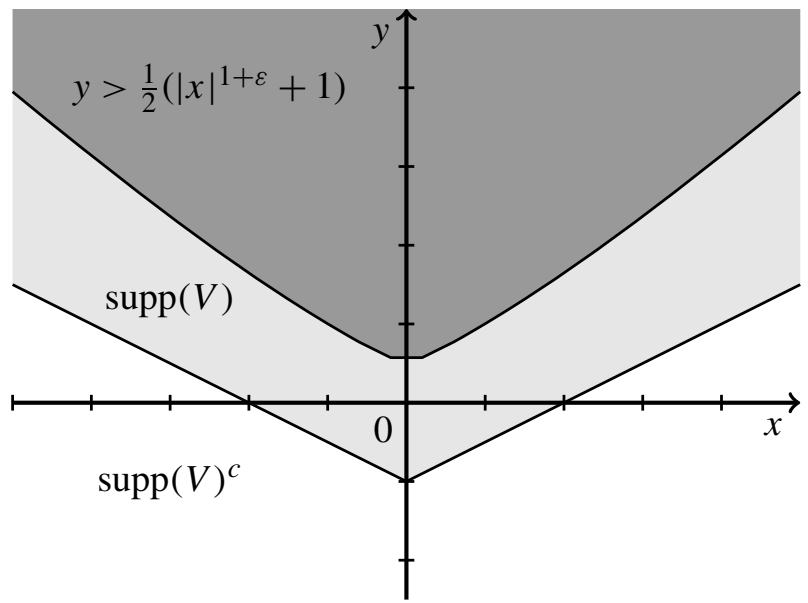

FIG. 1. The support of the harmonic function $V$ in Example 2.11.

Since the walk $\left(y+S_{n}\right)_{n \geq 1}$ can increase at each step with positive probability, it follows that $\mathbb{P}\left(y+S_{n_{0}}>\gamma, \tau_{y}>n_{0}\right)>0$ if and only if $\mathbb{P}\left(\tau_{y}>1\right)=\mathbb{P}\left(y+X_{1}>\right.$ $0)>0$. Thus, $[0,+\infty) \subseteq(-\max \operatorname{supp}(\boldsymbol{\mu}),+\infty)=\mathscr{D}_{\gamma}=\operatorname{supp}(V)$, for every $\gamma>$ 0 , where $\boldsymbol{\mu}$ is the common law of $X_{n}$ and $\operatorname{supp}(\boldsymbol{\mu})$ is its support.

The following example is intended to illustrate Remark 2.8.

EXAMPLE 2.11. Consider the following special case of the one dimensional stochastic recursion: $X_{n+1}=a_{n+1} X_{n}+b_{n+1}$ where $\left(a_{i}\right)_{i \geq 1}$ and $\left(b_{i}\right)_{i \geq 1}$ are two independent sequences of i.i.d. random variables. In this example, we consider that the law of $a_{i}$ is $\frac{1}{2} \delta_{\{-1 / 2\}}+\frac{1}{2} \delta_{\{1 / 2\}}$ and that of $b_{i}$ is uniform on $[-1,1]$. The state space $\mathbb{X}$ is $\mathbb{R}$. The functions $N$ and $N_{l}$ are given by $N(x)=|x|^{1+\varepsilon}$ for some $\varepsilon>0$, and $N_{l}(x)=N(x) \phi_{l}(|x|)$ with $\phi_{l}$ defined by (A.4). The Banach space satisfying M1-M5 is constructed in Section A (see also [18]). One can verify that the domain of positivity of the function $V$ is $\operatorname{supp}(V)=\left\{(x, y) \in \mathbb{R}^{2}: y>-\frac{|x|}{2}-1\right\}=\mathscr{D}_{\gamma}$, for all $\gamma>0$. Obviously, $\left\{(x, y) \in \mathbb{X} \times \mathbb{R}: y>\frac{1}{2}\left(1+|x|^{1+\varepsilon}\right)\right\} \subseteq \operatorname{supp}(V)$; see Figure 1.

The next example is intended to show that the inequality of point 2 of Theorem 2.3 is attained.

EXAMPLE 2.12. Consider the Markov walk $\left(X_{n}\right)_{n \geq 0}$ living on the finite state space $\mathbb{X}:=\{-1 ; 1 ;-3 ; 7 / 6\}$ with the transition probabilities given in Figure 2 . Suppose that $f$ is the identity function on $\mathbb{X}$. It is easy to see that the assumptions stated in Remark 3.10 of Section 3.3 are satisfied and thereby so are Hypotheses M1-M5. In particular, M4 holds with $N=N_{l}=0$ for any $l \geq 1$. Now, when 


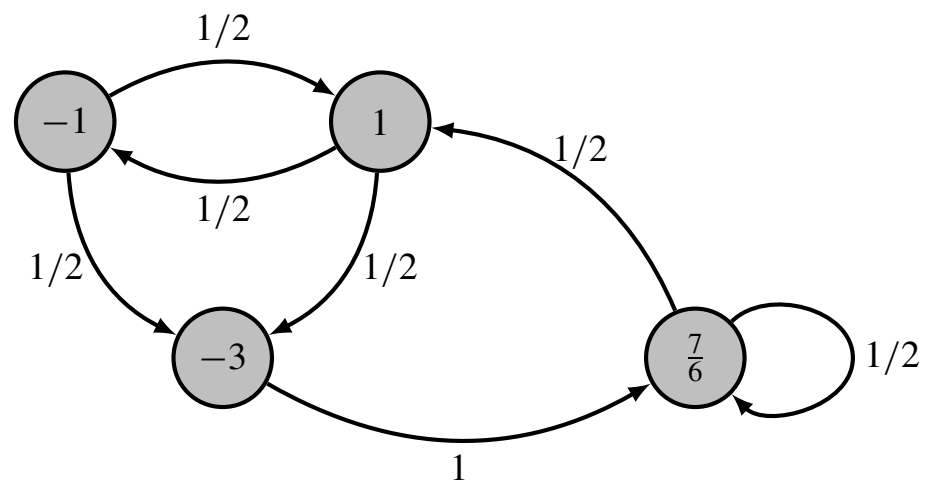

FIG. 2. Transition probabilities of the Markov chain in Example 2.12.

$x=1$ and $y \in(1,3]$ or when $x=-1$ and $y \in(-1,2]$, one can check that the Markov walk $y+S_{n}$ stays positive if and only if the values of the variables $X_{i}$ alternate between 1 and -1 and therefore, for such starting points $(x, y)$, we have $\mathbb{P}_{x}\left(\tau_{y}>n\right)=\left(\frac{1}{2}\right)^{n}$. This shows that, when the random variables $\left(X_{n}\right)_{n \geq 1}$ form a Markov chain, the survival probability $\mathbb{P}_{x}\left(\tau_{y}>n\right)$ has an asymptotic behaviour different from that in the independent case where it can be either equivalent to $\frac{c_{x, y}}{\sqrt{n}}$ or 0 .

In this example, we can make explicit the support of the function $V$. Since $N=0$, the function $V$ is positive if and only if there exists an integer $n \geq 1$ such that $\mathbb{P}_{x}\left(y+S_{n}>\gamma, \tau_{y}>n\right)>0$ for a $\gamma$ large enough. This is possible only if the chain can reach the state $X_{n}=7 / 6$ within a trajectory of $\left(y+S_{k}\right)_{n \geq k \geq 1}$ which stays positive, that is, $\mathbb{P}_{x}\left(X_{n}=7 / 6, \tau_{y}>n\right)>0$. Consequently,

$$
\begin{aligned}
\operatorname{supp}(V) & =\{-1\} \times(2,+\infty) \cup\{1\} \times(3,+\infty) \cup\{-3,7 / 6\} \times(-7 / 6,+\infty) \\
& =\mathscr{D}_{3}=\left\{(x, y) \in \mathbb{X} \times \mathbb{R}: \exists n \geq 1, \mathbb{P}_{x}\left(y+S_{n}>3, \tau_{y}>n\right)>0\right\} .
\end{aligned}
$$

To sum up, this model presents the three possible asymptotic behaviours of $\mathbb{P}_{x}\left(\tau_{y}>n\right)$ : for any $(x, y) \in \operatorname{supp}(V)=\{-1\} \times(2,+\infty) \cup\{1\} \times(3,+\infty) \cup$ $\{-3,7 / 6\} \times(-7 / 6,+\infty)$,

$$
\mathbb{P}_{x}\left(\tau_{y}>n\right) \underset{n \rightarrow+\infty}{\sim} \frac{2 V(x, y)}{\sqrt{2 \pi n} \sigma},
$$

for any $(x, y) \in\{-1\} \times(-1,2] \cup\{1\} \times(1,3]$ and $n \geq 1$,

$$
\mathbb{P}_{x}\left(\tau_{y}>n\right)=\left(\frac{1}{2}\right)^{n}
$$

for any $(x, y) \in\{-1\} \times(-\infty,-1] \cup\{1\} \times(-\infty, 1] \cup\{-3,7 / 6\} \times(-\infty,-7 / 6]$ and $n \geq 1$,

$$
\mathbb{P}_{x}\left(\tau_{y}>n\right)=0
$$


3. Applications. We illustrate the results of Section 2 by considering three particular models.

3.1. Affine random walk in $\mathbb{R}^{d}$ conditioned to stay in a half-space. Let $d \geq 1$ be an integer and $\left(g_{n}\right)_{n \geq 1}=\left(A_{n}, B_{n}\right)_{n \geq 1}$ be a sequence of i.i.d. random elements in $\operatorname{GL}(d, \mathbb{R}) \times \mathbb{R}^{d}$ following the same distribution $\boldsymbol{\mu}$. Let $\left(X_{n}\right)_{n \geq 0}$ be the Markov chain on $\mathbb{R}^{d}$ defined by

$$
X_{0}=x \in \mathbb{R}^{d}, \quad X_{n+1}=A_{n+1} X_{n}+B_{n+1}, \quad n \geq 1 .
$$

Set $S_{n}=\sum_{k=1}^{n} f\left(X_{k}\right), n \geq 1$, where the function $f(x)=\langle u, x\rangle$ is the projection of the vector $x \in \mathbb{R}^{d}$ on the direction defined by the vector $u \in \mathbb{R}^{d} \backslash\{0\}$. For any $y \in$ $\mathbb{R}$, consider the first time when the random walk $\left(y+S_{n}\right)_{n \geq 1}$ becomes nonpositive:

$$
\tau_{y}=\inf \left\{k \geq 1: y+S_{k} \leq 0\right\} .
$$

This stopping time coincides with the entry time of the affine walk $\left(\sum_{k=1}^{n} X_{k}\right)_{n \geq 0}$ in the closed half-subspace $\left\{s \in \mathbb{R}^{d}:\langle u, s\rangle \leq-y\right\}$.

Introduce the following hypothesis.

HyPOTHESIS 3.1. 1. There exists a constant $\delta>0$, such that

$$
\mathbb{E}\left(\left\|A_{1}\right\|^{2+2 \delta}\right)<+\infty, \quad \mathbb{E}\left(\left|B_{1}\right|^{2+2 \delta}\right)<+\infty
$$

and

$$
k(\delta)=\lim _{n \rightarrow+\infty} \mathbb{E}^{1 / n}\left(\left\|A_{n} A_{n-1} \cdots A_{1}\right\|^{2+2 \delta}\right)<1 .
$$

2. There is no proper affine subspace of $\mathbb{R}^{d}$ which is invariant with respect to all the elements of the support of $\boldsymbol{\mu}$.

3. For any vector $v_{0} \in \mathbb{R}^{d} \backslash\{0\}$,

$$
\mathbb{P}\left({ }^{t} A_{1}^{-1} v_{0}={ }^{t} A_{2}^{-1} v_{0}\right)<1,
$$

where ${ }^{t} A$ is the transpose of $A$, for any $A \in \mathrm{GL}(d, \mathbb{R})$.

4. The vector $B_{1}$ is centred: $\mathbb{E}\left(B_{1}\right)=0$.

Proposition 3.2. Under Hypothesis 3.1, Theorems 2.2-2.5 hold true.

Proposition 3.2 is proved in Appendix A where we construct an appropriate Banach space $\mathscr{B}$ and show that Hypotheses M1-M5 are satisfied with $N(x)=|x|^{1+\varepsilon}$, for some $\varepsilon>0$ and with $N_{l}(x)=N(x) \phi_{l}(|x|)$, where $\phi_{l}$ is defined by (A.4).

REMARK 3.3. The set $\operatorname{supp}(V)$ depends on the law of $\left(A_{i}, B_{i}\right)$. In the case when $A_{i}$ are independent of $B_{i}$ and the support of the law of $\left\langle u, B_{i}\right\rangle$ contains a sequence converging to $+\infty$, one can verify that $\operatorname{supp}(V)=\mathbb{R}^{d} \times \mathbb{R}$. 
3.2. Two components Markov chains in compact sets under the doeblin-fortet condition. Let $\left(X, d_{X}\right)$ be a compact metric space, $\mathscr{C}(X)$ and $\mathscr{L}(X)$ be the spaces of continuous and Lipschitz complex functions on $X$, respectively. Define

$$
|h|_{\infty}=\sup _{x \in X}|h(x)|, \quad \forall h \in \mathscr{C}(X)
$$

and

$$
[h]_{X}=\sup _{\substack{(x, y) \in X \\ x \neq y}} \frac{|h(x)-h(y)|}{d_{X}(x, y)}, \quad \forall h \in \mathscr{L}(X) .
$$

We endow $\mathscr{C}(X)$ with the uniform norm $|\cdot|_{\infty}$ and $\mathscr{L}(X)$ with the norm $|\cdot|_{\mathscr{L}}=$ $|\cdot|_{\infty}+[\cdot]_{X}$, respectively. Consider the space $\mathbb{X}:=X \times X$ with the metric $d_{\mathbb{X}}$ on $\mathbb{X}$ defined by $d_{\mathbb{X}}\left(\left(x_{1}, x_{2}\right),\left(y_{1}, y_{2}\right)\right)=d_{X}\left(x_{1}, y_{1}\right)+d_{X}\left(x_{2}, y_{2}\right)$, for any $\left(x_{1}, x_{2}\right)$ and $\left(y_{1}, y_{2}\right)$ in $\mathbb{X}$. Denote by $\mathscr{L}(\mathbb{X})$ the space of the Lipschitz complex function on $\mathbb{X}$ endowed with the norm $\|\cdot\|_{\mathscr{L}}=\|\cdot\|_{\infty}+[\cdot]_{\mathbb{X}}$, where

$$
\|h\|_{\infty}=\sup _{x \in \mathbb{X}}|h(x)|, \quad \forall h \in \mathscr{C}(\mathbb{X})
$$

and

$$
[h]_{\mathbb{X}}=\sup _{\substack{(x, y) \in \mathbb{X} \\ x \neq y}} \frac{|h(x)-h(y)|}{d_{\mathbb{X}}(x, y)}, \quad \forall h \in \mathscr{L}(\mathbb{X}) .
$$

Following Guivarc' $h$ and Hardy [21], consider a Markov chain $\left(\chi_{n}\right)_{n \geq 0}$ on $X$ with transition probability $P$. Let $\left(X_{n}\right)_{n \geq 0}$ be the Markov chain on $\mathbb{X}$ defined by $X_{n}=$ $\left(\chi_{n-1}, \chi_{n}\right), n \geq 1$ and $X_{0}=\left(0, \chi_{0}\right)$ : its transition probability is given by

$$
\mathbf{P}\left(\left(x_{1}, x_{2}\right), \mathrm{d} y_{1} \times \mathrm{d} y_{2}\right)=\boldsymbol{\delta}_{x_{2}}\left(\mathrm{~d} y_{1}\right) P\left(x_{2}, \mathrm{~d} y_{2}\right) .
$$

For a fixed real function $f$ on $\mathbb{X}$, let $S_{n}:=\sum_{k=1}^{n} f\left(X_{n}\right)$ be the associated Markov walk and, for any $y \in \mathbb{R}$, let $\tau_{y}:=\inf \left\{n \geq 1: y+S_{n} \leq 0\right\}$ be the associated exit time.

In order to apply the results stated in the previous section, we need some hypotheses on the function $f$ and the operator $P$ on $\mathscr{C}(X)$ defined by $P h(x)=$ $\int_{X} h(y) P(x, \mathrm{~d} y)$ for any $x \in X$ and any $h \in \mathscr{C}(X)$.

Hypothesis 3.4. 1. For any $h$ in $\mathscr{C}(X)$, respectively in $\mathscr{L}(X)$, the function $P h$ is an element of $\mathscr{C}(X)$, respectively of $\mathscr{L}(X)$.

2. There exist constants $n_{0} \geq 1,0<\rho<1$ and $C>0$ such that, for any function $h \in \mathscr{L}(X)$, we have

$$
\left|P^{n_{0}} h\right|_{\mathscr{L}} \leq \rho|h|_{\mathscr{L}}+C|h|_{\infty}
$$

3. The unique eigenvalue of $P$ of modulus 1 is 1 and the associated eigenspace is generated by the function $e: x \mapsto 1$, that is, if there exist $\theta \in \mathbb{R}$ and $h \in \mathscr{L}(X)$ such that $P h=\mathrm{e}^{i \theta} h$, then $h$ is constant and $\mathrm{e}^{i \theta}=1$. 
Under Hypothesis 3.4, one can check that conditions (a), (b), (c) and (d) of Chapter 3 in Norman [28] hold true and we can apply the theorem of Ionescu Tulcea and Marinescu [25] (see also [21]). Coupling this theorem with point 3 of Hypothesis 3.4 we obtain the following proposition.

Proposition 3.5. 1. There exists a unique $P$-invariant probability $v$ on $X$.

2. For any $n \geq 1$ and $h \in \mathscr{L}(X)$,

$$
P^{n} h=v(h)+R^{n} h,
$$

where $R$ is an operator on $\mathscr{L}(X)$ with a spectral radius $r(R)<1$.

Suppose that $f$ and $v$ satisfy the following hypothesis.

Hypothesis 3.6. 1. The function $f$ belongs to $\mathscr{L}(\mathbb{X})$.

2. The function $f$ is centred, in the sense that

$$
\int_{\mathbb{X}} f(x, y) P(x, \mathrm{~d} y) v(\mathrm{~d} x)=0 .
$$

3. The function $f$ is nondegenerated, that means that there is no function $h \in$ $\mathscr{L}(X)$ such that

$$
f(x, y)=h(x)-h(y),
$$

for $P_{v}$-almost all $(x, y)$, where $P_{v}(\mathrm{~d} x \times \mathrm{d} y)=P(x, \mathrm{~d} y) v(\mathrm{~d} x)$.

Assuming Hypotheses 3.4 and 3.6, Guivarc'h and Hardy [21] have established that the sequence $\left(S_{n} / \sqrt{n}\right)_{n \geq 1}$ converges weakly to a centred Gaussian random variable of variance $\sigma^{2}>0$, under the probability $\mathbb{P}_{x}$ generated by the finite dimensional distributions of the Markov chain $\left(X_{n}\right)_{n \geq 0}$ starting at $X_{0}=x$, for any $x \in X$. Moreover, under the same hypotheses, we show in Appendix B that M1-M5 are satisfied with $N=N_{l}=0$, thereby proving the following assertion.

Proposition 3.7. Under Hypotheses 3.4 and 3.6, Theorems 2.2-2.5 hold true.

3.3. Markov chains in compact sets under spectral gap assumptions. In this section, we give sufficient conditions in order that a Markov chain with values in a compact set satisfy conditions M1-M5.

Let $(\mathbb{X}, d)$ be a compact metric space and $\left(X_{n}\right)_{n \geq 0}$ be a Markov chain living in $\mathbb{X}$. Denote by $\mathbf{P}$ the transition probability of $\left(X_{n}\right)_{n \geq 0}$ and by $\mathscr{C}(\mathbb{X})$ the Banach algebra of the continuous complex functions on $\mathbb{X}$ endowed with the uniform norm

$$
|h|_{\infty}=\sup _{x \in \mathbb{X}}|h(x)|, \quad h \in \mathscr{C}(\mathbb{X}) .
$$

Consider a real function $f$ defined on $\mathbb{X}$, the transition operator $\mathbf{P}$ on $\mathscr{C}(\mathbb{X})$ associated to the transition probability of $\left(X_{n}\right)_{n \geq 0}$ and the unit function $e$ defined on $\mathbb{X}$ by $e(x)=1$, for any $x \in \mathbb{X}$. 
Hypothesis 3.8. 1. For any $h \in \mathscr{C}(\mathbb{X})$, the function $\mathbf{P} h$ is an element of $\mathscr{C}(\mathbb{X})$.

2. The operator $\mathbf{P}$ has a unique invariant probability $\boldsymbol{v}$.

3. For any $n \geq 1$,

$$
\mathbf{P}^{n}=\Pi+Q^{n}
$$

where $\Pi$ is the one-dimensional projector on $\mathscr{C}(\mathbb{X})$ defined by $\Pi(h)=v(h)$ e, for any $h \in \mathscr{C}(\mathbb{X}), Q$ is an operator on $\mathscr{C}(\mathbb{X})$ of spectral radius $r(Q)<1$ satisfying $\Pi Q=Q \Pi=0$.

4. The function $f$ belongs to $\mathscr{C}(\mathbb{X})$ and is $\boldsymbol{v}$-centred, that is, $\boldsymbol{v}(f)=0$.

5. The function $f$ is nondegenerated, that is there is no function $h \in \mathscr{C}(\mathbb{X})$ such that

$$
f\left(X_{1}\right)=h\left(X_{0}\right)-h\left(X_{1}\right), \quad \mathbb{P}_{\boldsymbol{v}} \text {-a.s., }
$$

where $\mathbb{P}_{\boldsymbol{v}}$ is the probability generated by the finite dimensional distributions of the Markov chain $\left(X_{n}\right)_{n \geq 0}$ when the initial law of $X_{0}$ is $\boldsymbol{v}$.

Consider the Markov walk $S_{n}=\sum_{k=1}^{n} f\left(X_{k}\right)$. It is well known, that under Hypothesis 3.8 the normalized sum $S_{n} / \sqrt{n}$ converges in law to a centred normal distribution of variance $\sigma^{2}>0$ with respect to the probability $\mathbb{P}_{x}$ generated by the finite dimensional distributions of the Markov chain $\left(X_{n}\right)_{n \geq 0}$ starting at $X_{0}=x$, for any $x \in \mathbb{X}$.

Proposition 3.9. Under Hypothesis 3.8, Theorems 2.2-2.5 hold true.

All the elements of the proof are contained in the proof of Proposition 3.7 (see Appendix B), which therefore is left to the reader. In particular, Hypothesis M4 holds with $N=N_{l}=0$.

REMARK 3.10. As a special example of the compact case, consider the Markov chain $\left(X_{n}\right)_{n \geq 1}$ taking values in a finite space $\mathbb{X}$. Assume that $\left(X_{n}\right)_{n \geq 1}$ is aperiodic and irreducible with transition matrix $\mathbf{P}$. Let $f$ be a finite function on $\mathbb{X}$. We shall verify Hypothesis 3.8 . The Banach space $\mathscr{B}$ consists of all finite real functions on $\mathbb{X}$, therefore condition 1 is obvious. Moreover, there is a unique invariant measure $\boldsymbol{v}$, which proves condition 2. According to PerronFrobenius theorem, the transition matrix $\mathbf{P}$ admits 1 as the only simple eigenvalue of modulus 1 , which implies condition 3. Assume in addition that $\boldsymbol{v}(f)=0$ (which is condition 4) and that there exists a path $x_{0}, \ldots, x_{n}$ in $\mathbb{X}$ such that $\mathbf{P}\left(x_{0}, x_{1}\right)>0, \ldots, \mathbf{P}\left(x_{n-1}, x_{n}\right)>0, \mathbf{P}\left(x_{n}, x_{0}\right)>0$ and $f\left(x_{0}\right)+\cdots+f\left(x_{n}\right) \neq 0$ (which implies condition 5). Thus, all the conclusions of Theorems 2.2-2.5 hold true.

\section{Preliminary statements.}

4.1. Results for the Brownian motion. Let $\left(B_{t}\right)_{t>0}$ be the standard Brownian motion with values in $\mathbb{R}$ living on the probability space $(\Omega, \mathscr{F}, \mathbb{P})$. Define the exit 
time

$$
\tau_{y}^{b m}=\inf \left\{t \geq 0: y+\sigma B_{t} \leq 0\right\},
$$

where $\sigma>0$. The following affirmations are due to Lévy [27].

LEMMA 4.1. For any $y>0,0 \leq a \leq b$ and $n \geq 1$,

$$
\mathbb{P}\left(\tau_{y}^{b m}>n, y+\sigma B_{n} \in[a, b]\right)=\frac{1}{\sqrt{2 \pi n} \sigma} \int_{a}^{b}\left(\mathrm{e}^{-\frac{(s-y)^{2}}{2 n \sigma^{2}}}-\mathrm{e}^{-\frac{(s+y)^{2}}{2 n \sigma^{2}}}\right) \mathrm{d} s .
$$

LEMMA 4.2. 1. For any $y>0$,

$$
\mathbb{P}\left(\tau_{y}^{b m}>n\right) \leq c \frac{y}{\sqrt{n}} .
$$

2. For any sequence of real numbers $\left(\theta_{n}\right)_{n \geq 0}$ such that $\theta_{n} \underset{n \rightarrow+\infty}{\longrightarrow} 0$,

$$
\sup _{y \in\left[0 ; \theta_{n} \sqrt{n}\right]}\left(\frac{\mathbb{P}\left(\tau_{y}^{b m}>n\right)}{\frac{2 y}{\sqrt{2 \pi n} \sigma}}-1\right)=O\left(\theta_{n}^{2}\right) .
$$

4.2. Strong approximation. Under Hypotheses M1-M5 it is proved in [19] that there is a version of the Markov walk $\left(S_{n}\right)_{n \geq 0}$ and of the standard Brownian motion $\left(B_{t}\right)_{t \geq 0}$ living on the same probability space which are close enough in the following sense.

Proposition 4.3. There exists $\varepsilon_{0}>0$ such that, for any $\varepsilon \in\left(0, \varepsilon_{0}\right]$, without loss of generality one can reconstruct the sequence $\left(S_{n}\right)_{n \geq 0}$ together with a continuous time Brownian motion $\left(B_{t}\right)_{t \in \mathbb{R}_{+}}$, such that for any $x \in \mathbb{X}$ and $n \geq 1$,

$$
\mathbb{P}_{x}\left(\sup _{0 \leq t \leq 1}\left|S_{\lfloor t n\rfloor}-\sigma B_{t n}\right|>n^{1 / 2-\varepsilon}\right) \leq \frac{c_{\varepsilon}}{n^{\varepsilon}}(1+N(x)),
$$

where $\sigma$ is defined in point 2 of Proposition 2.1.

In the original result the right-hand side in (4.2) is $c_{\varepsilon} n^{-\varepsilon}\left(1+\mu_{\alpha}(x)+\right.$ $\left.\left\|\boldsymbol{\delta}_{x}\right\|_{\mathscr{B}^{\prime}}\right)^{\alpha} \leq c_{\varepsilon} n^{-\varepsilon}(1+N(x))^{\alpha}$ with $\alpha>2$, by point 1 of the Hypothesis M5. To obtain the result of Proposition 4.3 it suffices to take the power $1 / \alpha$ on the both sides and to use the obvious inequality $p<p^{1 / \alpha}$, for $p \in[0,1]$.

Using Proposition 4.3, we easily deduce the following result.

COROLlaRY 4.4. There exists $\varepsilon_{0}>0$ such that, for any $\varepsilon \in\left(0, \varepsilon_{0}\right), x \in \mathbb{R}$ and $n \geq 1$,

$$
\sup _{t \in \mathbb{R}}\left|\mathbb{P}_{x}\left(\frac{S_{n}}{\sqrt{n}} \leq t\right)-\int_{-\infty}^{t} \mathrm{e}^{-\frac{u^{2}}{2 \sigma^{2}}} \frac{\mathrm{d} u}{\sqrt{2 \pi} \sigma}\right| \leq \frac{c_{\varepsilon}}{n^{\varepsilon}}(1+N(x)) .
$$


5. Martingale approximation and related assertions. In this section, we construct an approximating martingale for the Markov walk $\left(S_{n}\right)_{n \geq 0}$, which will be used subsequently to define the harmonic function. We also state some useful properties.

Consider $\Theta$ the real valued function defined on $\mathbb{X}$ by

$$
\Theta(x)=f(x)+\sum_{k=1}^{+\infty} \mathbf{P}^{k} f(x), \quad \forall x \in \mathbb{X} .
$$

It is well known that $\Theta$ is the solution of the Poisson equation

$$
\Theta-\mathbf{P} \Theta=f .
$$

For any $x \in \mathbb{X}$, let

$$
r(x)=\mathbf{P} \Theta(x)=\Theta(x)-f(x)=\sum_{k=1}^{+\infty} \mathbf{P}^{k} f(x) .
$$

Following Gordin [17], define the process $\left(M_{n}\right)_{n \geq 0}$ by setting $M_{0}=0$ and, for any $n \geq 1$,

$$
M_{n}=\sum_{k=1}^{n}\left[\Theta\left(X_{k}\right)-\mathbf{P} \Theta\left(X_{k-1}\right)\right]=\sum_{k=1}^{n}\left[\Theta\left(X_{k}\right)-r\left(X_{k-1}\right)\right] .
$$

For any $x \in \mathbb{X}$, we have that $\left(M_{n}\right)_{n \geq 0}$ is a zero mean $\mathbb{P}_{x}$-martingale with respect to the natural filtration $\left(\mathscr{F}_{n}\right)_{n \geq 0}$. Denote by $\xi_{n}$ the increments of the martingale $\left(M_{n}\right)_{n \geq 0}$ : for any $n \geq 1$,

$$
\xi_{n}:=\Theta\left(X_{n}\right)-r\left(X_{n-1}\right) .
$$

In the sequel it will be convenient to consider the martingale $\left(z+M_{n}\right)_{n \geq 1}$ starting at

$$
z=y+r(x) .
$$

The reason for this is the following approximation which is an easy consequence of the definition of the martingale $\left(z+M_{n}\right)_{n \geq 1}$ : for any $x \in \mathbb{X}$ and $y \in \mathbb{R}$, we have

$$
z+M_{n}=y+S_{n}+r\left(X_{n}\right) \text {. }
$$

From (2.6), we deduce the following assertion.

LEMMA 5.1. The functions $\Theta$ and $r$ exist on $\mathbb{X}$ and for any $x \in \mathbb{X}$,

$$
|\Theta(x)| \leq c(1+N(x)) \text { and }|r(x)| \leq c(1+N(x)) .
$$

We show that the moments of order $p \in[1, \alpha]$ of the martingale $\left(M_{n}\right)_{n \geq 0}$ are bounded. 
LEMMA 5.2. 1. For any $p \in[1, \alpha], x \in \mathbb{X}$ and $n \geq 1$,

$$
\mathbb{E}_{x}^{1 / p}\left(\left|M_{n}\right|^{p}\right) \leq c_{p} \sqrt{n}(1+N(x)) .
$$

2. For any $x \in \mathbb{X}$ and $n \geq 1$,

$$
\mathbb{E}_{x}\left(\left|M_{n}\right|\right) \leq c(\sqrt{n}+N(x)) .
$$

PROOF. First, we control the increments $\xi_{n}$. By Lemma 5.1, for any $n \geq 1$,

$$
\left|\xi_{n}\right| \leq c\left(1+N\left(X_{n}\right)+N\left(X_{n-1}\right)\right) .
$$

So, using point 1 of Hypothesis M4 and (2.2), for any $n \geq 1$,

$$
\begin{aligned}
\mathbb{E}_{x}^{1 / p}\left(\left|\xi_{n}\right|^{p}\right) & \leq c_{p}(1+N(x)) \quad \forall p \in[1, \alpha], \\
\mathbb{E}_{x}\left(\left|\xi_{n}\right|\right) & \leq c+c \mathrm{e}^{-c n} N(x) .
\end{aligned}
$$

Proof of claim 1. By Burkholder's inequality, for $2<p \leq \alpha$,

$$
\mathbb{E}_{x}^{1 / p}\left(\left|M_{n}\right|^{p}\right) \leq c_{p} \mathbb{E}_{x}^{1 / p}\left(\left(\sum_{k=1}^{n} \xi_{k}^{2}\right)^{p / 2}\right)
$$

Using Hölder's inequality with the exponents $u=p / 2>1$ and $v=\frac{p}{p-2}$, we obtain

$$
\mathbb{E}_{x}^{1 / p}\left(\left|M_{n}\right|^{p}\right) \leq c_{p} \mathbb{E}_{x}^{1 / p}\left[\left(\sum_{k=1}^{n} \xi_{k}^{2 u}\right)^{\frac{p}{2 u}} n^{\frac{p}{2 v}}\right]=c_{p} n^{\frac{p-2}{2 p}}\left(\sum_{k=1}^{n} \mathbb{E}_{x}\left[\left|\xi_{k}\right|^{p}\right]\right)^{1 / p}
$$

From (5.3), for any $p \in(2, \alpha]$,

$$
\mathbb{E}_{x}^{1 / p}\left(\left|M_{n}\right|^{p}\right) \leq c_{p} n^{\frac{p-2}{2 p}}\left(\sum_{k=1}^{n} c_{p}(1+N(x))^{p}\right)^{1 / p} \leq c_{p} \sqrt{n}(1+N(x)) .
$$

Using the Jensen inequality for $p \in[1,2]$, we obtain claim 1 .

Proof of claim 2. Consider $\varepsilon \in(0,1 / 2)$. By (5.4),

$$
\begin{aligned}
\mathbb{E}_{x}\left(\left|M_{n}\right|\right) & \leq \sum_{k=1}^{\left\lfloor n^{\varepsilon}\right\rfloor} \mathbb{E}_{x}\left(\left|\xi_{k}\right|\right)+\mathbb{E}_{x}\left(\left|M_{n}-M_{\left\lfloor n^{\varepsilon}\right\rfloor}\right|\right) \\
& \leq c n^{\varepsilon}+c N(x)+\mathbb{E}_{x}\left(\left|M_{n}-M_{\left\lfloor n^{\varepsilon}\right\rfloor}\right|\right) .
\end{aligned}
$$

Since $\left(X_{n}, M_{n}\right)_{n \geq 0}$ is a Markov chain, by the Markov property, claim 1 and (2.2),

$$
\begin{aligned}
\mathbb{E}_{x}\left(\left|M_{n}\right|\right) & \leq c n^{\varepsilon}+c N(x)+\mathbb{E}_{x}\left(\mathbb{E}\left(\left|M_{n}-M_{\left\lfloor n^{\varepsilon}\right\rfloor}\right| \mid \mathscr{F}_{\left\lfloor n^{\varepsilon}\right\rfloor}\right)\right) \\
& \leq c n^{\varepsilon}+c N(x)+\mathbb{E}_{x}\left[c\left(n-\left\lfloor n^{\varepsilon}\right\rfloor\right)^{1 / 2}\left(1+N\left(X_{\left\lfloor n^{\varepsilon}\right\rfloor}\right)\right)\right] \\
& \leq c \sqrt{n}+c_{\varepsilon} N(x) .
\end{aligned}
$$


A key point in the proof of the existence and of the positivity of the harmonic function is the introduction of the following stopping times. Let $T_{z}$ be the first time when the martingale $\left(z+M_{n}\right)_{n \geq 1}$ becomes nonpositive, and let $\hat{T}_{z}$ be the first time, after the time $\tau_{y}$, when the martingale $\left(z+M_{n}\right)_{n \geq 1}$ becomes nonpositive. Precisely, for any $x \in \mathbb{X}, z \in \mathbb{R}$ and $y=z-r(x)$, set

$$
T_{z}:=\inf \left\{k \geq 1: z+M_{k} \leq 0\right\} \quad \text { and } \quad \hat{T}_{z}:=\inf \left\{k \geq \tau_{y}: z+M_{k} \leq 0\right\} .
$$

The finiteness of the stopping times $\tau_{y}, T_{z}$ and $\hat{T}_{z}$ is proved in Lemmas 5.5, 5.6 and 5.7 below. Now we point out some elementary facts which will be helpful in the sequel. First, the stopping time $\hat{T}_{z}$ is such that $\tau_{y} \leq \hat{T}_{z}$ and $T_{z} \leq \hat{T}_{z}$. Since $\tau_{y}$ is the exit time of $\left(y+S_{n}\right)_{n \geq 0}$, by the Markov property,

$$
\mathbb{P}_{x}\left(\tau_{y}>n\right)=\int_{\mathbb{X} \times \mathbb{R}} \mathbb{P}_{x^{\prime}}\left(\tau_{y^{\prime}}>n-k\right) \mathbb{P}_{x}\left(X_{k} \in \mathrm{d} x^{\prime}, y+S_{k} \in \mathrm{d} y^{\prime}, \tau_{y}>k\right) .
$$

A similar expression holds true for $T_{z}$. Unfortunately, (5.7) does not hold for $\hat{T}_{z}$. Instead we have a more sophisticated expression given by the following lemma. We shall use repeatedly the same trick for more complicated functionals, as for example $\mathbb{E}_{x}\left(z+M_{n} ; \hat{T}_{z}>n\right)$.

LeMmA 5.3. For any $x \in \mathbb{X}, z \in \mathbb{R}, n \geq 1, k \leq n$ and $y=z-r(x)$,

$$
\begin{aligned}
& \mathbb{P}_{x}\left(\hat{T}_{z}>n\right) \\
& =\int_{\mathbb{X} \times \mathbb{R}} \mathbb{P}_{x^{\prime}}\left(\hat{T}_{z^{\prime}}>n-k\right) \mathbb{P}_{x}\left(X_{k} \in \mathrm{d} x^{\prime}, z+M_{k} \in \mathrm{d} z^{\prime}, \tau_{y}>k\right) \\
& \quad+\int_{\mathbb{X} \times \mathbb{R}} \mathbb{P}_{x^{\prime}}\left(T_{z^{\prime}}>n-k\right) \mathbb{P}_{x}\left(X_{k} \in \mathrm{d} x^{\prime}, z+M_{k} \in \mathrm{d} z^{\prime}, \tau_{y} \leq k, \hat{T}_{z}>k\right) .
\end{aligned}
$$

PROOF. Since $\hat{T}_{z} \geq \tau_{y}$, for any $k \leq n$, we have

$$
\mathbb{P}_{x}\left(\hat{T}_{z}>n\right)=\mathbb{P}_{x}\left(\tau_{y}>n\right)+\sum_{i=1}^{n-k} \mathbb{P}_{x}\left(\tau_{y}=i+k, \hat{T}_{z}>n\right)+\mathbb{P}_{x}\left(\tau_{y} \leq k, \hat{T}_{z}>n\right) .
$$

By the Markov property and (5.1), with $y^{\prime}=z^{\prime}-r\left(x^{\prime}\right)$,

$$
\begin{aligned}
\mathbb{P}_{x}\left(\hat{T}_{z}>n\right)= & \int_{\mathbb{X} \times \mathbb{R}} \mathbb{P}_{x^{\prime}}\left(\tau_{y^{\prime}}>n-k\right) \mathbb{P}_{x}\left(X_{k} \in \mathrm{d} x^{\prime}, z+M_{k} \in \mathrm{d} z^{\prime}, \tau_{y}>k\right) \\
& +\sum_{i=1}^{n-k} \int_{\mathbb{X} \times \mathbb{R}} \mathbb{P}_{x^{\prime}}\left(\tau_{y^{\prime}}=i, z^{\prime}+M_{i}>0, \ldots, z^{\prime}+M_{n-k}>0\right) \\
& \times \mathbb{P}_{x}\left(X_{k} \in \mathrm{d} x^{\prime}, z+M_{k} \in \mathrm{d} z^{\prime}, \tau_{y}>k\right) \\
& +\int_{\mathbb{X} \times \mathbb{R}} \mathbb{P}_{x^{\prime}}\left(T_{z^{\prime}}>n-k\right) \mathbb{P}_{x}\left(X_{k} \in \mathrm{d} x^{\prime}, z+M_{k} \in \mathrm{d} z^{\prime}, \tau_{y} \leq k,\right. \\
& \left.z+M_{\tau_{y}}>0, \ldots, z+M_{k}>0\right) .
\end{aligned}
$$

Putting together the first two terms we get the result. 
The following lemma will be useful in the next sections.

LEMMA 5.4. For any $x \in \mathbb{X}, z \in \mathbb{R}$, the sequence $\left(\left(z+M_{n}\right) \mathbb{1}_{\left\{\hat{T}_{z}>n\right\}}\right)_{n \geq 0}$ is a $\mathbb{P}_{x}$-submartingale.

PRoOF. Let $x \in \mathbb{X}, z \in \mathbb{R}$. For any $n \geq 0$,

$$
\begin{aligned}
\mathbb{E}_{x}\left(\left(z+M_{n+1}\right) \mathbb{1}_{\left\{\hat{T}_{z}>n+1\right\}} \mid \mathscr{F}_{n}\right) & \\
& =\mathbb{E}_{x}\left(\left(z+M_{n+1}\right) \mathbb{1}_{\left\{\hat{T}_{z}>n\right\}} \mid \mathscr{F}_{n}\right)-\mathbb{E}_{x}\left(\left(z+M_{n+1}\right) \mathbb{1}_{\left\{\hat{T}_{z}=n+1\right\}} \mid \mathscr{F}_{n}\right) \\
& =\left(z+M_{n}\right) \mathbb{1}_{\left\{\hat{T}_{z}>n\right\}}-\mathbb{E}_{x}\left(\left(z+M_{\hat{T}_{z}}\right) \mathbb{1}_{\left\{\hat{T}_{z}=n+1\right\}} \mid \mathscr{F}_{n}\right) .
\end{aligned}
$$

By the definition of $\hat{T}_{z}$ we have $z+M_{\hat{T}_{z}} \leq 0 \mathbb{P}_{x}$-a.s. and the result follows.

We end this section by proving the finiteness of $\tau_{y}, T_{z}$ and $\hat{T}_{z}$.

LEMMA 5.5. For any $x \in \mathbb{X}$ and $y \in \mathbb{R}$,

$$
\tau_{y}<+\infty \quad \mathbb{P}_{x} \text {-a.s. }
$$

PROOF. Let $x \in \mathbb{X}$. Assume first that $y>0$. Since $\left\{\tau_{y}>n\right\}$ is a nonincreasing sequence of events,

$$
\mathbb{P}_{x}\left(\tau_{y}=+\infty\right)=\lim _{n \rightarrow+\infty} \mathbb{P}_{x}\left(\tau_{y}>n\right)=\lim _{n \rightarrow+\infty} \mathbb{P}_{x}\left(y+S_{k}>0, \forall k \leq n\right) .
$$

Using Proposition 4.3,

$$
\mathbb{P}_{x}\left(y+S_{k}>0, \forall k \leq n\right) \leq \frac{c_{\varepsilon}}{n^{\varepsilon}}(1+N(x))+\mathbb{P}\left(\tau_{y+n^{1 / 2-\varepsilon}}^{b m}>n\right) .
$$

Thus, by point 1 of Lemma 4.2,

$$
\mathbb{P}_{x}\left(\tau_{y}>n\right) \leq \frac{c_{\varepsilon}}{n^{\varepsilon}}(1+N(x))+c \frac{y+n^{1 / 2-\varepsilon}}{\sqrt{n}} \leq \frac{c_{\varepsilon}}{n^{\varepsilon}}(1+y+N(x)) .
$$

When $y \leq 0$, we have, for any $y^{\prime}>0, \mathbb{P}_{x}\left(\tau_{y}>n\right) \leq \mathbb{P}_{x}\left(\tau_{y^{\prime}}>n\right)$. Taking the limit when $y^{\prime} \rightarrow 0$, we obtain that

$$
\mathbb{P}_{x}\left(\tau_{y}>n\right) \leq \frac{c_{\varepsilon}}{n^{\varepsilon}}(1+N(x)) .
$$

From (5.8) and (5.9) it follows that, for any $y \in \mathbb{R}$,

$$
\mathbb{P}_{x}\left(\tau_{y}>n\right) \leq \frac{c_{\varepsilon}}{n^{\varepsilon}}(1+\max (y, 0)+N(x)) .
$$

Taking the limit as $n \rightarrow+\infty$, we conclude that $\tau_{y}<+\infty \mathbb{P}_{x}$-a.s.

The same result can be obtained for the exit time $T_{z}$ of the martingale $(z+$ $\left.M_{n}\right)_{n \geq 0}$. 
LEMMA 5.6. For any $x \in \mathbb{X}$ and $z \in \mathbb{R}$,

$$
T_{z}<+\infty \quad \mathbb{P}_{x} \text {-a.s. }
$$

PRoOf. Let $x \in \mathbb{X}, z \in \mathbb{R}$ and $y=z-r(x)$. Assume first that $y=z-r(x)>$ 0 . Following the proof of Lemma 5.5,

$$
\mathbb{P}_{x}\left(T_{z}=+\infty\right)=\lim _{n \rightarrow+\infty} \mathbb{P}_{x}\left(z+M_{k}>0, \forall k \leq n\right)
$$

By (5.1) the martingale $\left(z+M_{n}\right)_{n \geq 0}$ is relied to the Markov walk $\left(y+S_{n}\right)_{n \geq 0}$, which gives

$$
\begin{aligned}
\mathbb{P}_{x}\left(z+M_{k}>0, \forall k \leq n\right) \leq & \mathbb{P}_{x}\left(y+S_{k}>-n^{1 / 2-\varepsilon}, \forall k \leq n\right) \\
& +\mathbb{P}_{x}\left(\max _{1 \leq k \leq n}\left|r\left(X_{k}\right)\right|>n^{1 / 2-\varepsilon}\right) .
\end{aligned}
$$

On the one hand, in the same way as in the proof of Lemma 5.5,

$$
\mathbb{P}_{x}\left(y+S_{k}>-n^{1 / 2-\varepsilon}, \forall k \leq n\right) \leq \frac{c_{\varepsilon}}{n^{\varepsilon}}(1+N(x))+\mathbb{P}_{x}\left(\tau_{y+2 n^{1 / 2-\varepsilon}}^{b m}>n\right)
$$

On the other hand, using Lemma 5.1, for $n$ large enough,

$$
\mathbb{P}_{x}\left(\max _{1 \leq k \leq n}\left|r\left(X_{k}\right)\right|>n^{1 / 2-\varepsilon}\right) \leq \sum_{k=1}^{\left\lfloor n^{\varepsilon}\right\rfloor} \mathbb{E}_{x}\left(\frac{c N\left(X_{k}\right)}{n^{1 / 2-\varepsilon}}\right)+\sum_{k=\left\lfloor n^{\varepsilon}\right\rfloor+1}^{n} \mathbb{E}_{x}\left(\frac{c N_{l}\left(X_{k}\right)}{n^{1 / 2-\varepsilon}}\right),
$$

where $l=c n^{1 / 2-\varepsilon}$. So, using (2.3) and taking $\varepsilon \leq \min \left(\frac{1}{6}, \frac{\beta}{2(3+\beta)}\right)$, we obtain

$$
\mathbb{P}_{x}\left(\max _{1 \leq k \leq n}\left|r\left(X_{k}\right)\right|>n^{1 / 2-\varepsilon}\right) \leq \frac{c_{\varepsilon}}{n^{\varepsilon}}(1+N(x)) .
$$

Putting together (5.11), (5.12) and (5.13) and using point 1 of Lemma 4.2, we have, for $z>r(x)$,

$$
\mathbb{P}_{x}\left(T_{z}>n\right) \leq \frac{c_{\varepsilon}}{n^{\varepsilon}}(1+N(x))+c \frac{y+2 n^{1 / 2-\varepsilon}}{\sqrt{n}} \leq \frac{c_{\varepsilon}}{n^{\varepsilon}}(1+\max (z, 0)+N(x)) .
$$

Since $z \mapsto T_{z}$ is nondecreasing, we obtain the same bound for any $z \in \mathbb{R}$,

$$
\mathbb{P}_{x}\left(T_{z}>n\right) \leq \frac{c_{\varepsilon}}{n^{\varepsilon}}(1+\max (z, 0)+N(x)) .
$$

Taking the limit as $n \rightarrow+\infty$ we conclude that $T_{z}<+\infty \mathbb{P}_{x}$-a.s.

LEMMA 5.7. For any $x \in \mathbb{X}$ and $z \in \mathbb{R}$,

$$
\hat{T}_{z}<+\infty \quad \mathbb{P}_{x} \text {-a.s. }
$$


PROOF. In order to apply Lemmas 5.5 and 5.6, we write, with $y=z-r(x)$,

$$
\begin{aligned}
\mathbb{P}_{x}\left(\hat{T}_{z}>n\right) \leq & \mathbb{P}_{x}\left(\tau_{y}>\lfloor n / 2\rfloor\right) \\
+ & \int_{\mathbb{X} \times \mathbb{R}} \mathbb{P}_{x^{\prime}}\left(T_{z^{\prime}}>n-\lfloor n / 2\rfloor\right) \mathbb{P}_{x}\left(X_{\lfloor n / 2\rfloor} \in \mathrm{d} x^{\prime},\right. \\
& \left.z+M_{\lfloor n / 2\rfloor} \in \mathrm{d} z^{\prime}, \tau_{y} \leq\lfloor n / 2\rfloor, \hat{T}_{z}>\lfloor n / 2\rfloor\right) .
\end{aligned}
$$

Using (5.10), (5.14) and the definition of $y$, we have

$$
\begin{aligned}
\mathbb{P}_{x}\left(\hat{T}_{z}>n\right) \leq & \frac{c_{\varepsilon}}{n^{\varepsilon}}(1+\max (y, 0)+N(x)) \\
& +\frac{c_{\varepsilon}}{n^{\varepsilon}} \mathbb{E}_{x}\left(1+z+M_{\lfloor n / 2\rfloor}+N\left(X_{\lfloor n / 2\rfloor}\right) ; \tau_{y} \leq\lfloor n / 2\rfloor, \hat{T}_{z}>\lfloor n / 2\rfloor\right) .
\end{aligned}
$$

By point 1 of Hypothesis M4,

$$
\begin{aligned}
\mathbb{P}_{x}\left(\hat{T}_{z}>n\right) \leq & \frac{c_{\varepsilon}}{n^{\varepsilon}}(1+\max (y, 0)+N(x))+\frac{c_{\varepsilon}}{n^{\varepsilon}} \mathbb{E}_{x}\left(z+M_{\lfloor n / 2\rfloor} ; \hat{T}_{z}>\lfloor n / 2\rfloor\right) \\
& -\frac{c_{\varepsilon}}{n^{\varepsilon}} \mathbb{E}_{x}\left(z+M_{\lfloor n / 2\rfloor} ; \tau_{y}>\lfloor n / 2\rfloor\right) .
\end{aligned}
$$

Using (5.1), we see that on the event $\left\{\tau_{y}>\lfloor n / 2\rfloor\right\}$ we have $z+M_{\lfloor n / 2\rfloor}>r\left(X_{\lfloor n / 2\rfloor}\right)$. Then, by Lemma 5.1 and point 1 of Hypothesis M4,

$$
\mathbb{P}_{x}\left(\hat{T}_{z}>n\right) \leq \frac{c_{\varepsilon}}{n^{\varepsilon}}(1+\max (y, 0)+N(x))+\frac{c_{\varepsilon}}{n^{\varepsilon}} \mathbb{E}_{x}\left(z+M_{\lfloor n / 2\rfloor} ; \hat{T}_{z}>\lfloor n / 2\rfloor\right) .
$$

Using Lemma 6.4, we have

$$
\mathbb{P}_{x}\left(\hat{T}_{z}>n\right) \leq \frac{c_{\varepsilon}}{n^{\varepsilon}}(1+\max (y, 0)+N(x)) .
$$

Finally, we conclude that

$$
\mathbb{P}_{x}\left(\hat{T}_{z}=+\infty\right)=\lim _{n \rightarrow+\infty} \mathbb{P}_{x}\left(\hat{T}_{z}>n\right)=0
$$

6. Integrability of the killed martingale and of the killed Markov walk. The goal of this section is to show that the expectations of the martingale $(z+$ $\left.M_{n}\right)_{n \geq 0}$ killed at $\hat{T}_{z}$ and of the Markov walk $\left(y+S_{n}\right)_{n \geq 0}$ killed at $\tau_{y}$ are bounded uniformly in $n$.

We start by establishing two auxiliary bounds of order $n^{1 / 2-2 \varepsilon}$ for the expectations of the martingale $\left(z+M_{n}\right)_{n \geq 0}$ killed at $T_{z}$ or at $\hat{T}_{z}$.

LEMMA 6.1. There exists $\varepsilon_{0}>0$ such that, for any $\varepsilon \in\left(0, \varepsilon_{0}\right), x \in \mathbb{X}, z \in \mathbb{R}$ and $n \geq 1$, it holds

$$
\mathbb{E}_{x}\left(z+M_{n} ; T_{z}>n\right) \leq \max (z, 0)+c_{\varepsilon}\left(n^{1 / 2-2 \varepsilon}+N(x)\right) .
$$


PROOF. Using the fact that $\left(M_{n}\right)_{n \geq 0}$ is a zero mean martingale and the optional stopping theorem,

$$
\mathbb{E}_{x}\left(z+M_{n} ; T_{z}>n\right)=z-\mathbb{E}_{x}\left(z+M_{n} ; T_{z} \leq n\right)=z-\mathbb{E}_{x}\left(z+M_{T_{z}} ; T_{z} \leq n\right) .
$$

By the definition of $T_{z}$, on the event $\left\{T_{z}>1\right\}$, we have

$$
\xi_{T_{z}}=z+M_{T_{z}}-\left(z+M_{T_{z}-1}\right)<z+M_{T_{z}} \leq 0 .
$$

Using this inequality and (5.2), we obtain

$$
\begin{aligned}
\mathbb{E}_{x}(z & \left.+M_{n} ; T_{z}>n\right) \\
& \leq z \mathbb{P}_{x}\left(T_{z}>1\right)+\mathbb{E}_{x}\left(\left|\xi_{1}\right| ; T_{z}=1\right)+\mathbb{E}_{x}\left(\left|\xi_{T_{z}}\right| ; 1<T_{z} \leq n\right) \\
& \leq \max (z, 0)+c \mathbb{E}_{x}\left(1+N\left(X_{T_{z}}\right)+N\left(X_{T_{z}-1}\right) ; T_{z} \leq n\right) .
\end{aligned}
$$

We bound $\mathbb{E}_{x}\left(N\left(X_{T_{z}}\right) ; T_{z} \leq n\right)$ as follows. Let $\varepsilon$ be a real number in $(0,1 / 6)$ and set $l=\left\lfloor n^{1 / 2-2 \varepsilon}\right\rfloor$. Using point 1 of Hypothesis M4, we write

$$
\begin{aligned}
\mathbb{E}_{x}\left(N\left(X_{T_{z}}\right) ; T_{z} \leq n\right) & \leq n^{1 / 2-2 \varepsilon}+\mathbb{E}_{x}\left(N\left(X_{T_{z}}\right) ; N\left(X_{T_{z}}\right)>n^{1 / 2-2 \varepsilon}, T_{z} \leq n\right) \\
& \leq n^{1 / 2-2 \varepsilon}+\sum_{k=1}^{\left\lfloor n^{\varepsilon}\right\rfloor} \mathbb{E}_{x}\left(N\left(X_{k}\right)\right)+\sum_{k=\left\lfloor n^{\varepsilon}\right\rfloor+1}^{n} \mathbb{E}_{x}\left(N_{l}\left(X_{k}\right)\right) .
\end{aligned}
$$

By (2.2) and (2.3),

$$
\mathbb{E}_{x}\left(N\left(X_{T_{z}}\right) ; T_{z} \leq n\right) \leq c n^{1 / 2-2 \varepsilon}+c N(x)+\frac{c n}{l^{1+\beta}}+c \mathrm{e}^{-c n^{\varepsilon}}(1+N(x)) .
$$

Choosing $\varepsilon<\min \left(\frac{\beta}{4(2+\beta)}, \frac{1}{6}\right)$, we find that

$$
\mathbb{E}_{x}\left(N\left(X_{T_{z}}\right) ; T_{z} \leq n\right) \leq c_{\varepsilon} n^{1 / 2-2 \varepsilon}+c_{\varepsilon} N(x) .
$$

In the same manner, we obtain that $\mathbb{E}_{x}\left(N\left(X_{T_{z}-1}\right) ; T_{z} \leq n\right) \leq c_{\varepsilon} n^{1 / 2-2 \varepsilon}+c_{\varepsilon} N(x)$. Consequently, from (6.2) and (6.1), we conclude the assertion of the lemma.

LEMmA 6.2. There exists $\varepsilon_{0}>0$ such that, for any $\varepsilon \in\left(0, \varepsilon_{0}\right), x \in \mathbb{X}, z \in \mathbb{R}$ and $n \geq 1$, we have

$$
\mathbb{E}_{x}\left(z+M_{n} ; \hat{T}_{z}>n\right) \leq \max (z, 0)+c_{\varepsilon}\left(n^{1 / 2-2 \varepsilon}+n^{2 \varepsilon} N(x)\right) .
$$

PROOF. Let $\varepsilon$ be a real number in $(0,1 / 4)$ and $n \geq 1$. Denoting $z_{+}:=z+$ $n^{1 / 2-2 \varepsilon}$ we have,

$$
\begin{aligned}
\mathbb{E}_{x}\left(z+M_{n} ; \hat{T}_{z}>n\right)= & \underbrace{\mathbb{E}_{x}\left(z+M_{n} ; T_{z_{+}} \leq n, \hat{T}_{z}>n\right)}_{=: J_{1}} \\
& +\underbrace{\mathbb{E}_{x}\left(z+M_{n} ; T_{z_{+}}>n, \hat{T}_{z}>n\right)}_{=: J_{2}} .
\end{aligned}
$$


Bound of $J_{1}$. Recall that $y=z-r(x)$. Using the definition of $\hat{T}_{z}$, we can see that on the event $\left\{\tau_{y} \leq k, \hat{T}_{z}>k\right\}$ it holds $z_{+}+M_{k}>z+M_{k}>0$. So $\mathbb{P}_{x}\left(\tau_{y} \leq\right.$ $\left.k, \hat{T}_{z}>k, T_{z_{+}}=k\right)=0$. Using this fact and the Markov property, in the same way as in the proof of Lemma 5.3,

$$
\begin{aligned}
J_{1}= & \sum_{k=1}^{n} \int_{\mathbb{X} \times \mathbb{R}} \mathbb{E}_{x^{\prime}}\left(z^{\prime}+M_{n-k} ; \hat{T}_{z^{\prime}}>n-k\right) \\
& \times \mathbb{P}_{x}\left(X_{k} \in \mathrm{d} x^{\prime}, z+M_{k} \in \mathrm{d} z^{\prime}, \tau_{y}>k, T_{z_{+}}=k\right) .
\end{aligned}
$$

Since $z+M_{T_{z_{+}}}<0$, using point 2 of Lemma 5.2, we have

$$
J_{1} \leq c \mathbb{E}_{x}\left(\sqrt{n}+N\left(X_{T_{z_{+}}}\right) ; \tau_{y}>T_{z_{+}}, T_{z_{+}} \leq n\right) .
$$

By the approximation (5.1), on the event $\left\{\tau_{y}>T_{z_{+}}\right\}$, it holds

$$
r\left(X_{T_{z_{+}}}\right)=z+M_{T_{z_{+}}}-\left(y+S_{T_{z_{+}}}\right)<-n^{1 / 2-2 \varepsilon} .
$$

Therefore, by Lemma 5.1,

$$
\begin{aligned}
J_{1} & \leq c n^{2 \varepsilon} \mathbb{E}_{x}\left(\left|r\left(X_{T_{z_{+}}}\right)\right|+N\left(X_{T_{z_{+}}}\right) ;\left|r\left(X_{T_{z_{+}}}\right)\right|>n^{1 / 2-2 \varepsilon}, T_{z_{+}} \leq n\right) \\
& \leq c n^{2 \varepsilon}+c n^{2 \varepsilon} \mathbb{E}_{x}\left(N\left(X_{T_{z_{+}}}\right) ; T_{z_{+}} \leq n\right) .
\end{aligned}
$$

Choosing $\varepsilon$ small enough, by (6.2),

$$
J_{1} \leq c n^{2 \varepsilon}+c_{\varepsilon} n^{2 \varepsilon}\left(n^{1 / 2-4 \varepsilon}+N(x)\right) \leq c_{\varepsilon} n^{1 / 2-2 \varepsilon}+c_{\varepsilon} n^{2 \varepsilon} N(x) .
$$

Bound of $J_{2}$. By Lemma 6.1, there exists $\varepsilon_{0}>0$ such that, for any $\varepsilon \in\left(0, \varepsilon_{0}\right)$,

$$
J_{2} \leq \mathbb{E}_{x}\left(z_{+}+M_{n} ; T_{z_{+}}>n\right) \leq \max (z, 0)+c_{\varepsilon} n^{1 / 2-2 \varepsilon}+c_{\varepsilon} N(x) .
$$

Inserting this bound and (6.4) into (6.3), for any $\varepsilon \in\left(0, \varepsilon_{0}\right)$, we deduce the assertion of the lemma.

Let $v_{n}$ be the first time when the martingale $z+M_{n}$ exceeds $n^{1 / 2-\varepsilon}$ : for any $n \geq 1, \varepsilon \in(0,1 / 2)$ and $z \in \mathbb{R}$,

$$
v_{n}=v_{n, \varepsilon, z}:=\min \left\{k \geq 1: z+M_{k}>n^{1 / 2-\varepsilon}\right\} .
$$

The control on the joint law of $v_{n}$ and $\hat{T}_{z}$ is given by the following lemma.

LEMMA 6.3. There exists $\varepsilon_{0}>0$ such that, for any $\varepsilon \in\left(0, \varepsilon_{0}\right), \delta>0, x \in \mathbb{X}$, $z \in \mathbb{R}$ and $n \geq 1$,

$$
\mathbb{P}_{x}\left(v_{n}>\delta n^{1-\varepsilon}, \hat{T}_{z}>\delta n^{1-\varepsilon}\right) \leq c_{\varepsilon, \delta} \mathrm{e}^{-c_{\varepsilon, \delta} n^{\varepsilon}}(1+N(x)) .
$$


PROOF. Let $\varepsilon \in(0,1 / 4)$ and $\delta>0$. Without loss of generally, we assume that $n \geq c_{\varepsilon, \delta}$, where $c_{\varepsilon, \delta}$ is large enough. Set $K:=\left\lfloor n^{\varepsilon} / 2\right\rfloor$. We split the interval $\left[1, \delta n^{1-\varepsilon}\right]$ by subintervals of length $l:=\left\lfloor\delta n^{1-2 \varepsilon}\right\rfloor$. For any $k \in\{1, \ldots, K\}$, introduce the event $A_{k, z}:=\left\{\max _{1 \leq k^{\prime} \leq k}\left(z+M_{k^{\prime} l}\right) \leq n^{1 / 2-\varepsilon}\right\}$. Then

$$
\mathbb{P}_{x}\left(v_{n}>\delta n^{1-\varepsilon}, \hat{T}_{z}>\delta n^{1-\varepsilon}\right) \leq \mathbb{P}_{x}\left(A_{2 K, z}, \hat{T}_{z}>2 K l\right) .
$$

By the Markov property, as in the proof of Lemma 5.3, with $y=z-r(x)$, we have

$$
\begin{aligned}
& \mathbb{P}_{x}\left(A_{2 K, z}, \hat{T}_{z}>2 K l\right) \\
& =\int_{\mathbb{X} \times \mathbb{R}} \mathbb{P}_{x^{\prime}}\left(A_{2, z^{\prime}}, \hat{T}_{z^{\prime}}>2 l\right) \mathbb{P}_{x}\left(X_{2(K-1) l} \in \mathrm{d} x^{\prime}, z+M_{2(K-1) l} \in \mathrm{d} z^{\prime},\right. \\
& \left.\quad A_{2(K-1), z}, \tau_{y}>2(K-1) l\right) \\
& \quad+\int_{\mathbb{X} \times \mathbb{R}} \mathbb{P}_{x^{\prime}}\left(A_{2, z^{\prime}}, T_{z^{\prime}}>2 l\right) \mathbb{P}_{x}\left(X_{2(K-1) l} \in \mathrm{d} x^{\prime}, z+M_{2(K-1) l} \in \mathrm{d} z^{\prime},\right. \\
& \\
& \left.\quad A_{2(K-1), z}, \tau_{y} \leq 2(K-1) l, \hat{T}_{z}>2(K-1) l\right) .
\end{aligned}
$$

Moreover, with $y^{\prime}=z^{\prime}-r\left(x^{\prime}\right)$, we write also that

$$
\begin{aligned}
& \mathbb{P}_{x^{\prime}}\left(A_{2, z^{\prime}}, \hat{T}_{z^{\prime}}>2 l\right) \\
& =\int_{\mathbb{X} \times \mathbb{R}} \mathbb{P}_{x^{\prime \prime}}\left(A_{1, z^{\prime \prime}}, \hat{T}_{z^{\prime \prime}}>l\right) \mathbb{P}_{x^{\prime}}\left(X_{l} \in \mathrm{d} x^{\prime \prime}, z^{\prime}+M_{l} \in \mathrm{d} z^{\prime \prime}, A_{1, z^{\prime}}, \tau_{y^{\prime}}>l\right) \\
& \quad+\int_{\mathbb{X} \times \mathbb{R}} \mathbb{P}_{x^{\prime \prime}}\left(A_{1, z^{\prime \prime}}, T_{z^{\prime \prime}}>l\right) \\
& \quad \times \mathbb{P}_{x^{\prime}}\left(X_{l} \in \mathrm{d} x^{\prime \prime}, z^{\prime}+M_{l} \in \mathrm{d} z^{\prime \prime}, A_{1, z^{\prime}}, \tau_{y^{\prime}} \leq l, \hat{T}_{z^{\prime}}>l\right) .
\end{aligned}
$$

Bound of $\mathbb{P}_{x^{\prime \prime}}\left(A_{1, z^{\prime \prime}}, \hat{T}_{z^{\prime \prime}}>l\right)$. Note that on the event $\left\{\tau_{y^{\prime}}>l\right\}$ we have $z^{\prime}+M_{l}-r\left(X_{l}\right)=y^{\prime}+S_{l}>0$. Consequently, in the first integral of the righthand side of (6.8), the integration over $\mathbb{X} \times \mathbb{R}$ can be replaced by the integration over $\left\{\left(x^{\prime \prime}, z^{\prime \prime}\right) \in \mathbb{X} \times \mathbb{R}: z^{\prime \prime}-r\left(x^{\prime \prime}\right)>0\right\}$. Therefore it is enough to bound $\mathbb{P}_{x^{\prime \prime}}\left(A_{1, z^{\prime \prime}}, \hat{T}_{z^{\prime \prime}}>l\right)$ for $x^{\prime \prime}$ and $z^{\prime \prime}$ satisfying $y^{\prime \prime}=z^{\prime \prime}-r\left(x^{\prime \prime}\right)>0$. Using (5.1) we have,

$$
\begin{aligned}
\mathbb{P}_{x^{\prime \prime}}\left(A_{1, z^{\prime \prime}}, \hat{T}_{z^{\prime \prime}}>l\right) \leq & \mathbb{P}_{x^{\prime \prime}}\left(y^{\prime \prime}+S_{l} \leq 2 n^{1 / 2-\varepsilon},\left|r\left(X_{l}\right)\right| \leq n^{1 / 2-\varepsilon}\right) \\
& +\mathbb{P}_{x^{\prime \prime}}\left(\left|r\left(X_{l}\right)\right|>n^{1 / 2-\varepsilon}\right) .
\end{aligned}
$$

Therefore, there exists a constant $c_{\varepsilon, \delta}$ such that

$$
\mathbb{P}_{x^{\prime \prime}}\left(A_{1, z^{\prime \prime}}, \hat{T}_{z^{\prime \prime}}>l\right) \leq \mathbb{P}_{x^{\prime \prime}}\left(\frac{S_{l}}{\sqrt{l}} \leq c_{\varepsilon, \delta}\right)+\mathbb{E}_{x^{\prime \prime}}\left(\frac{\left|r\left(X_{l}\right)\right|}{n^{1 / 2-\varepsilon}}\right) .
$$


Using Corollary 4.4 and Lemma 5.1, there exists $\varepsilon_{0} \in(0,1 / 4)$, such that, for any $\varepsilon \in\left(0, \varepsilon_{0}\right)$,

$$
\begin{aligned}
& \mathbb{P}_{x^{\prime \prime}}\left(A_{1, z^{\prime \prime}}, \hat{T}_{z^{\prime \prime}}>l\right) \\
& \quad \leq \int_{-\infty}^{c_{\varepsilon, \delta}} \mathrm{e}^{-\frac{u^{2}}{2 \sigma^{2}}} \frac{\mathrm{d} u}{\sqrt{2 \pi} \sigma}+\frac{c_{\varepsilon}}{l^{\varepsilon}}\left(1+N\left(x^{\prime \prime}\right)\right)+\frac{c}{n^{1 / 2-\varepsilon}} \mathbb{E}_{x^{\prime \prime}}\left(1+N\left(X_{l}\right)\right) .
\end{aligned}
$$

Using point 1 of Hypothesis M4 and the fact that $l^{\varepsilon} \geq n^{\varepsilon / 2} / c_{\varepsilon, \delta}$ for $\varepsilon<1 / 4$, we have,

$$
\mathbb{P}_{x^{\prime \prime}}\left(A_{1, z^{\prime \prime}}, \hat{T}_{z^{\prime \prime}}>l\right) \leq q_{\varepsilon, \delta}+\frac{c_{\varepsilon, \delta}}{n^{\varepsilon / 2}}\left(1+N\left(x^{\prime \prime}\right)\right)
$$

with $q_{\varepsilon, \delta}:=\int_{-\infty}^{c_{\varepsilon, \delta}} \mathrm{e}^{-\frac{u^{2}}{2 \sigma^{2}}} \frac{\mathrm{d} u}{\sqrt{2 \pi} \sigma}<1$.

Bound of $\mathbb{P}_{x^{\prime \prime}}\left(A_{1, z^{\prime \prime}}, T_{z^{\prime \prime}}>l\right)$. On the event $\left\{T_{z^{\prime \prime}}>l\right\}$ we have $z^{\prime \prime}+M_{l}>0$. Using (5.1) and Corollary 4.4, in the same way as in the proof of the bound (6.9), we obtain

$$
\begin{aligned}
\mathbb{P}_{x^{\prime \prime}}\left(A_{1, z^{\prime \prime}}, T_{z^{\prime \prime}}>l\right) & \leq \mathbb{P}_{x^{\prime \prime}}\left(0<z^{\prime \prime}+M_{l} \leq n^{1 / 2-\varepsilon}\right) \\
& \leq \int_{\frac{-y^{\prime \prime}}{\sqrt{l}}-c_{\varepsilon, \delta}}^{\frac{-y^{\prime \prime}}{\sqrt{l}}+c_{\varepsilon, \delta}} \mathrm{e}^{-\frac{u^{2}}{2 \sigma^{2}}} \frac{\mathrm{d} u}{\sqrt{2 \pi} \sigma}+\frac{c_{\varepsilon, \delta}}{n^{\varepsilon / 2}}\left(1+N\left(x^{\prime \prime}\right)\right) \\
& \leq q_{\varepsilon, \delta}+\frac{c_{\varepsilon, \delta}}{n^{\varepsilon / 2}}\left(1+N\left(x^{\prime \prime}\right)\right)
\end{aligned}
$$

Bound of $\mathbb{P}_{x^{\prime}}\left(A_{2, z^{\prime}}, \hat{T}_{z^{\prime}}>2 l\right)$. Inserting (6.9) and (6.10) into (6.8) and using (2.2), we have

$$
\begin{aligned}
\mathbb{P}_{x^{\prime}}\left(A_{2, z^{\prime}}, \hat{T}_{z^{\prime}}>2 l\right) & \leq q_{\varepsilon, \delta}+\frac{c_{\varepsilon, \delta}}{n^{\varepsilon / 2}}+\frac{c_{\varepsilon, \delta}}{n^{\varepsilon / 2}} \mathbb{E}_{x^{\prime}}\left(N\left(X_{l}\right)\right) \\
& \leq q_{\varepsilon, \delta}+\frac{c_{\varepsilon, \delta}}{n^{\varepsilon / 2}}+c_{\varepsilon, \delta} \mathrm{e}^{-c_{\varepsilon, \delta} n^{1-2 \varepsilon}} N\left(x^{\prime}\right)
\end{aligned}
$$

Bound of $\mathbb{P}_{x^{\prime}}\left(A_{2, z^{\prime}}, T_{z^{\prime}}>2 l\right)$. By the Markov property,

$$
\begin{aligned}
\mathbb{P}_{x^{\prime}}\left(A_{2, z^{\prime}}, T_{z^{\prime}}>2 l\right)= & \int_{\mathbb{X} \times \mathbb{R}} \mathbb{P}_{x^{\prime \prime}}\left(A_{1, z^{\prime \prime}}, T_{z^{\prime \prime}}>l\right) \\
& \times \mathbb{P}_{x^{\prime}}\left(X_{l} \in \mathrm{d} x^{\prime \prime}, z^{\prime}+M_{l} \in \mathrm{d} z^{\prime \prime}, A_{1, z^{\prime}}, T_{z^{\prime}}>l\right) .
\end{aligned}
$$

Using (6.10) to bound the probability inside the integral, we get

$$
\mathbb{P}_{x^{\prime}}\left(A_{2, z^{\prime}}, T_{z^{\prime}}>2 l\right) \leq q_{\varepsilon, \delta}+\frac{c_{\varepsilon, \delta}}{n^{\varepsilon / 2}}+c_{\varepsilon, \delta} \mathrm{e}^{-c_{\varepsilon, \delta} n^{1-2 \varepsilon}} N\left(x^{\prime}\right) .
$$


Inserting the bounds (6.11) and (6.12) into (6.7), we find that

$$
\begin{aligned}
\mathbb{P}_{x}\left(A_{2 K, z}, \hat{T}_{z}>2 K l\right) \leq & \left(q_{\varepsilon, \delta}+\frac{c_{\varepsilon, \delta}}{n^{\varepsilon / 2}}\right) \mathbb{P}_{x}\left(A_{2(K-1), z}, \hat{T}_{z}>2(K-1) l\right) \\
& +c_{\varepsilon, \delta} \mathrm{e}^{-c_{\varepsilon, \delta} n^{1-2 \varepsilon}}(1+N(x)) .
\end{aligned}
$$

Iterating this inequality, we get

$$
\begin{aligned}
& \mathbb{P}_{x}\left(A_{2 K, z}, \hat{T}_{z}>2 K l\right) \\
& \quad \leq\left(q_{\varepsilon, \delta}+\frac{c_{\varepsilon, \delta}}{n^{\varepsilon / 2}}\right)^{K}+c_{\varepsilon, \delta} \mathrm{e}^{-c_{\varepsilon, \delta} n^{1-2 \varepsilon}}(1+N(x)) \sum_{k=0}^{K-1}\left(q_{\varepsilon, \delta}+\frac{c_{\varepsilon, \delta}}{n^{\varepsilon / 2}}\right)^{k} .
\end{aligned}
$$

As $K=\left\lfloor n^{\varepsilon} / 2\right\rfloor$ and $q_{\varepsilon, \delta}<1$ it follows that, for $n$ large enough, $\left(q_{\varepsilon, \delta}+\frac{c_{\varepsilon, \delta}}{n^{\varepsilon / 2}}\right)^{K} \leq$ $c_{\varepsilon, \delta} \mathrm{e}^{-c_{\varepsilon, \delta} n^{\varepsilon}}$, which, in turn, implies

$$
\mathbb{P}_{x}\left(A_{2 K, z}, \hat{T}_{z}>2 K l\right) \leq c_{\varepsilon, \delta} \mathrm{e}^{-c_{\varepsilon, \delta} n^{\varepsilon}}(1+N(x)) .
$$

LEMMA 6.4. There exists $\varepsilon_{0}>0$ such that, for any $\varepsilon \in\left(0, \varepsilon_{0}\right), x \in \mathbb{X}, z \in \mathbb{R}$, $n \geq 2$ and any integer $k_{0} \in\{2, \ldots, n\}$,

$$
\mathbb{E}_{x}\left(z+M_{n} ; \hat{T}_{z}>n\right) \leq\left(1+\frac{c_{\varepsilon}}{k_{0}^{\varepsilon}}\right)(\max (z, 0)+c N(x))+c_{\varepsilon} k_{0}^{1 / 2} .
$$

PROOF. Set for brevity $u_{n}:=\mathbb{E}_{x}\left(z+M_{n} ; \hat{T}_{z}>n\right)$. By Lemma 5.4, the sequence $\left(u_{n}\right)_{n \geq 1}$ is nondecreasing. Let $\varepsilon \in(0,1 / 2)$. We shall prove below that, for $n \geq 2$,

$$
u_{n} \leq\left(1+\frac{c_{\varepsilon}}{n^{\varepsilon}}\right) u_{\left\lfloor n^{1-\varepsilon}\right\rfloor}+c_{\varepsilon} \mathrm{e}^{-c_{\varepsilon} n^{\varepsilon}}(1+N(x)) .
$$

Using Lemma 9.1 of [18], we obtain that for any $n \geq 2$ and $k_{0} \in\{2, \ldots, n\}$,

$$
u_{n} \leq\left(1+\frac{c_{\varepsilon}}{k_{0}^{\varepsilon}}\right) u_{k_{0}}+c_{\varepsilon} \mathrm{e}^{-c_{\varepsilon} k_{0}^{\varepsilon}}(1+N(x)) \text {. }
$$

Next, by point 2 of Lemma 5.2, $u_{k_{0}} \leq \mathbb{E}_{x}\left(\left|M_{k_{0}}\right|\right) \leq c\left(\sqrt{k_{0}}+N(x)\right)$, so that

$$
u_{n} \leq\left(1+\frac{c_{\varepsilon}}{k_{0}^{\varepsilon}}\right)(\max (z, 0)+c N(x))+c_{\varepsilon} k_{0}^{1 / 2},
$$

which proves Lemma 6.4.

Establishing (6.13) is rather tedious. In the proof, we make use of Lemmas 6.2 and 6.1. Consider the stopping time $v_{n}^{\varepsilon}:=v_{n}+\left\lfloor n^{\varepsilon}\right\rfloor$. Then,

$$
\begin{aligned}
u_{n} \leq \underbrace{\mathbb{E}_{x}\left(z+M_{n} ; \hat{T}_{z}>n, v_{n}^{\varepsilon}>\left\lfloor n^{1-\varepsilon}\right\rfloor\right)}_{=: J_{1}} \\
+\underbrace{\mathbb{E}_{x}\left(z+M_{n} ; \hat{T}_{z}>n, v_{n}^{\varepsilon} \leq\left\lfloor n^{1-\varepsilon}\right\rfloor\right)}_{=: J_{2}} .
\end{aligned}
$$


Bound of $J_{1}$. Set $m_{\varepsilon}=\left\lfloor n^{1-\varepsilon}\right\rfloor-\left\lfloor n^{\varepsilon}\right\rfloor$ and recall that $y=z-r(x)$. Using the fact that $\left\{v_{n}^{\varepsilon}>\left\lfloor n^{1-\varepsilon}\right\rfloor\right\}=\left\{v_{n}>m_{\varepsilon}\right\}$ and the Markov property, as in the proof of Lemma 5.3,

$$
\begin{aligned}
J_{1}= & \int_{\mathbb{X} \times \mathbb{R}} \mathbb{E}_{x^{\prime}}\left(z^{\prime}+M_{n-m_{\varepsilon}} ; \hat{T}_{z^{\prime}}>n-m_{\varepsilon}\right) \\
& \times \mathbb{P}_{x}\left(X_{m_{\varepsilon}} \in \mathrm{d} x^{\prime}, z+M_{m_{\varepsilon}} \in \mathrm{d} z^{\prime}, \tau_{y}>m_{\varepsilon}, v_{n}>m_{\varepsilon}\right) \\
& +\int_{\mathbb{X} \times \mathbb{R}} \mathbb{E}_{x^{\prime}}\left(z^{\prime}+M_{n-m_{\varepsilon}} ; T_{z^{\prime}}>n-m_{\varepsilon}\right) \\
& \times \mathbb{P}_{x}\left(X_{m_{\varepsilon}} \in \mathrm{d} x^{\prime}, z+M_{m_{\varepsilon}} \in \mathrm{d} z^{\prime}, \tau_{y} \leq m_{\varepsilon}, \hat{T}_{z}>m_{\varepsilon}, v_{n}>m_{\varepsilon}\right) .
\end{aligned}
$$

On the event $\left\{v_{n}>m_{\varepsilon}\right\}$, we have $z^{\prime}=z+M_{m_{\varepsilon}} \leq n^{1 / 2-\varepsilon} \leq n^{1 / 2}$. Moreover by point 2 of Lemma 5.2, $\mathbb{E}_{x^{\prime}}\left(\left|M_{n-m_{\varepsilon}}\right|\right) \leq c n^{1 / 2}+c N\left(x^{\prime}\right)$. Therefore,

$$
J_{1} \leq c \mathbb{E}_{x}\left(n^{1 / 2}+N\left(X_{m_{\varepsilon}}\right) ; \hat{T}_{z}>m_{\varepsilon}, v_{n}>m_{\varepsilon}\right)
$$

Set $m_{\varepsilon}^{\prime}=m_{\varepsilon}-\left\lfloor n^{\varepsilon}\right\rfloor=\left\lfloor n^{1-\varepsilon}\right\rfloor-2\left\lfloor n^{\varepsilon}\right\rfloor$. Using the Markov property and (2.2),

$$
\begin{aligned}
J_{1} & \leq c \int_{\mathbb{X}}\left[n^{1 / 2}+\mathbb{E}_{x^{\prime}}\left(N\left(X_{\left\lfloor n^{\varepsilon}\right\rfloor}\right)\right)\right] \mathbb{P}_{x}\left(X_{m_{\varepsilon}^{\prime}} \in \mathrm{d} x^{\prime}, \hat{T}_{z}>m_{\varepsilon}^{\prime}, v_{n}>m_{\varepsilon}^{\prime}\right) \\
& \leq c n^{1 / 2} \mathbb{P}_{x}\left(\hat{T}_{z}>m_{\varepsilon}^{\prime}, v_{n}>m_{\varepsilon}^{\prime}\right)+c \mathrm{e}^{-c n^{\varepsilon}} \mathbb{E}_{x}\left(N\left(X_{m_{\varepsilon}^{\prime}}\right)\right) .
\end{aligned}
$$

By Lemma 6.3 and point 1 of Hypothesis M4,

(6.15) $J_{1} \leq c_{\varepsilon} n^{1 / 2} \mathrm{e}^{-c_{\varepsilon} n^{\varepsilon}}(1+N(x))+c \mathrm{e}^{-c n^{\varepsilon}}(1+N(x)) \leq c_{\varepsilon} \mathrm{e}^{-c_{\varepsilon} n^{\varepsilon}}(1+N(x))$.

Bound of $J_{2}$. By the Markov property, as in the proof of Lemma 5.3, we have

$$
\begin{aligned}
J_{2}= & \sum_{k=1}^{\left\lfloor n^{1-\varepsilon}\right\rfloor} \int_{\mathbb{X} \times \mathbb{R}} \mathbb{E}_{x^{\prime}}\left(z^{\prime}+M_{n-k} ; \hat{T}_{z^{\prime}}>n-k\right) \\
& \times \mathbb{P}_{x}\left(X_{k} \in \mathrm{d} x^{\prime}, z+M_{k} \in \mathrm{d} z^{\prime}, \tau_{y}>k, v_{n}^{\varepsilon}=k\right) \\
& +\int_{\mathbb{X} \times \mathbb{R}} \mathbb{E}_{x^{\prime}}\left(z^{\prime}+M_{n-k} ; T_{z^{\prime}}>n-k\right) \\
& \times \mathbb{P}_{x}\left(X_{k} \in \mathrm{d} x^{\prime}, z+M_{k} \in \mathrm{d} z^{\prime}, \tau_{y} \leq k, \hat{T}_{z}>k, v_{n}^{\varepsilon}=k\right) .
\end{aligned}
$$

By Lemmas 6.2 and 6.1,

$$
\begin{gathered}
J_{2} \leq \underbrace{c_{\varepsilon} \mathbb{E}_{x}\left(n^{1 / 2-2 \varepsilon}+n^{2 \varepsilon} N\left(X_{v_{n}^{\varepsilon}}\right) ; \hat{T}_{z}>v_{n}^{\varepsilon}, v_{n}^{\varepsilon} \leq\left\lfloor n^{1-\varepsilon}\right\rfloor\right)}_{=: J_{21}} \\
+\underbrace{\mathbb{E}_{x}\left(\max \left(z+M_{v_{n}^{\varepsilon}}, 0\right) ; \hat{T}_{z}>v_{n}^{\varepsilon}, v_{n}^{\varepsilon} \leq\left\lfloor n^{1-\varepsilon}\right\rfloor\right)}_{=: J_{22}} .
\end{gathered}
$$


Bound of $J_{21}$. Using the Markov property and (2.2),

$$
\begin{aligned}
J_{21} & \leq c_{\varepsilon} \int_{\mathbb{X}} \mathbb{E}_{x^{\prime}}\left(n^{1 / 2-2 \varepsilon}+n^{2 \varepsilon} N\left(X_{\left\lfloor n^{\varepsilon}\right\rfloor}\right)\right) \mathbb{P}_{x}\left(X_{v_{n}} \in \mathrm{d} x^{\prime}, \hat{T}_{z}>v_{n}, v_{n} \leq\left\lfloor n^{1-\varepsilon}\right\rfloor\right) \\
& \leq c_{\varepsilon} \mathbb{E}_{x}\left(n^{1 / 2-2 \varepsilon}+\mathrm{e}^{-c_{\varepsilon} n^{\varepsilon}} N\left(X_{v_{n}}\right) ; \hat{T}_{z}>v_{n}, v_{n} \leq\left\lfloor n^{1-\varepsilon}\right\rfloor\right) .
\end{aligned}
$$

Again by (2.2),

$$
\begin{gathered}
\mathbb{E}_{x}\left(\mathrm{e}^{-c_{\varepsilon} n^{\varepsilon}} N\left(X_{v_{n}}\right) ; \hat{T}_{z}>v_{n}, v_{n} \leq\left\lfloor n^{1-\varepsilon}\right\rfloor\right) \\
\leq \mathrm{e}^{-c_{\varepsilon} n^{\varepsilon}} \sum_{k=1}^{\left\lfloor n^{1-\varepsilon}\right\rfloor} \mathbb{E}_{x}\left(N\left(X_{k}\right) ; v_{n}=k\right) \\
\leq c_{\varepsilon} \mathrm{e}^{-c_{\varepsilon} n^{\varepsilon}} n^{1-\varepsilon}(1+N(x)) .
\end{gathered}
$$

Therefore,

$$
J_{21} \leq \underbrace{c_{\varepsilon} \mathbb{E}_{x}\left(n^{1 / 2-2 \varepsilon} ; \hat{T}_{z}>v_{n}, v_{n} \leq\left\lfloor n^{1-\varepsilon}\right\rfloor\right)}_{=: J_{21}^{\prime}}+c_{\varepsilon} \mathrm{e}^{-c_{\varepsilon} n^{\varepsilon}}(1+N(x)) .
$$

By the definition of $v_{n}$, we have $n^{1 / 2-2 \varepsilon}<\frac{z+M_{v_{n}}}{n^{\varepsilon}}$. So

$$
J_{21}^{\prime} \leq \frac{c_{\varepsilon}}{n^{\varepsilon}} \mathbb{E}_{x}\left(z+M_{v_{n}} ; \hat{T}_{z}>v_{n}, v_{n} \leq\left\lfloor n^{1-\varepsilon}\right\rfloor\right) .
$$

Using Lemma 5.4,

$$
\begin{aligned}
J_{21}^{\prime} \leq & \frac{c_{\varepsilon}}{n^{\varepsilon}} \mathbb{E}_{x}\left(z+M_{\left\lfloor n^{1-\varepsilon}\right\rfloor} ; \hat{T}_{z}>\left\lfloor n^{1-\varepsilon}\right\rfloor\right) \\
& -\frac{c_{\varepsilon}}{n^{\varepsilon}} \underbrace{\mathbb{E}_{x}\left(z+M_{\left\lfloor n^{1-\varepsilon}\right\rfloor} ; \hat{T}_{z}>\left\lfloor n^{1-\varepsilon}\right\rfloor, v_{n}>\left\lfloor n^{1-\varepsilon}\right\rfloor\right)}_{=: J_{21}^{\prime \prime}} .
\end{aligned}
$$

Note that on the event $\left\{\tau_{y}>\left\lfloor n^{1-\varepsilon}\right\rfloor\right\}$, by (5.1), we have $z+M_{\left\lfloor n^{1-\varepsilon}\right\rfloor}>r\left(X_{\left\lfloor n^{1-\varepsilon}\right\rfloor}\right)$ while on the event $\left\{\tau_{y} \leq\left\lfloor n^{1-\varepsilon}\right\rfloor, \hat{T}_{z}>\left\lfloor n^{1-\varepsilon}\right\rfloor\right\}$ we have $z+M_{\left\lfloor n^{1-\varepsilon}\right\rfloor}>0$. Therefore, by the definition of $\hat{T}_{z}$,

$$
\begin{aligned}
-J_{21}^{\prime \prime} & \leq-\mathbb{E}_{x}\left(r\left(X_{\left\lfloor n^{1-\varepsilon}\right\rfloor}\right) ; \tau_{y}>\left\lfloor n^{1-\varepsilon}\right\rfloor, v_{n}>\left\lfloor n^{1-\varepsilon}\right\rfloor\right) \\
& \leq c \mathbb{E}_{x}\left(1+N\left(X_{\left\lfloor n^{1-\varepsilon}\right\rfloor}\right) ; \hat{T}_{z}>\left\lfloor n^{1-\varepsilon}\right\rfloor, v_{n}>\left\lfloor n^{1-\varepsilon}\right\rfloor\right) .
\end{aligned}
$$

Using the Markov property and (2.2),

$$
\begin{aligned}
-J_{21}^{\prime \prime} & \leq c \mathbb{E}_{x}\left(1+\mathrm{e}^{-c n^{\varepsilon}} N\left(X_{m_{\varepsilon}}\right) ; \hat{T}_{z}>m_{\varepsilon}, v_{n}>m_{\varepsilon}\right) \\
& \leq c \mathbb{P}_{x}\left(v_{n}>m_{\varepsilon}, \hat{T}_{z}>m_{\varepsilon}\right)+c \mathrm{e}^{-c n^{\varepsilon}}(1+N(x)) .
\end{aligned}
$$

By Lemma 6.3,

$$
-J_{21}^{\prime \prime} \leq c_{\varepsilon} \mathrm{e}^{-c_{\varepsilon} n^{\varepsilon}}(1+N(x))
$$


Putting together (6.20) and (6.19),

$$
J_{21}^{\prime} \leq \frac{c_{\varepsilon}}{n^{\varepsilon}} \mathbb{E}_{x}\left(z+M_{\left\lfloor n^{1-\varepsilon}\right\rfloor} ; \hat{T}_{z}>\left\lfloor n^{1-\varepsilon}\right\rfloor\right)+c_{\varepsilon} e^{-c_{\varepsilon} n^{\varepsilon}}(1+N(x)) .
$$

From (6.21) and (6.18), it follows that

$$
J_{21} \leq \frac{c_{\varepsilon}}{n^{\varepsilon}} \mathbb{E}_{x}\left(z+M_{\left\lfloor n^{1-\varepsilon}\right\rfloor} ; \hat{T}_{z}>\left\lfloor n^{1-\varepsilon}\right\rfloor\right)+c_{\varepsilon} \mathrm{e}^{-c_{\varepsilon} n^{\varepsilon}}(1+N(x)) .
$$

Bound of $J_{22}$. On the event $\left\{\hat{T}_{z}>v_{n}^{\varepsilon}, \tau_{y} \leq v_{n}^{\varepsilon}\right\}$ we have $z+M_{v_{n}^{\varepsilon}}>0$. Consequently,

$$
\begin{aligned}
J_{22}= & \mathbb{E}_{x}\left(z+M_{\nu_{n}^{\varepsilon}} ; \hat{T}_{z}>v_{n}^{\varepsilon}, v_{n}^{\varepsilon} \leq\left\lfloor n^{1-\varepsilon}\right\rfloor\right) \\
& +\mathbb{E}_{x}\left(\max \left(z+M_{\nu_{n}^{\varepsilon}}, 0\right)-\left(z+M_{\nu_{n}^{\varepsilon}}\right) ; \tau_{y}>v_{n}^{\varepsilon}, v_{n}^{\varepsilon} \leq\left\lfloor n^{1-\varepsilon}\right\rfloor\right) .
\end{aligned}
$$

By Lemma 5.4,

$$
\begin{aligned}
J_{22} \leq & \mathbb{E}_{x}\left(z+M_{\left\lfloor n^{1-\varepsilon}\right\rfloor} ; \hat{T}_{z}>\left\lfloor n^{1-\varepsilon}\right\rfloor\right) \\
& -\underbrace{\mathbb{E}_{x}\left(z+M_{\left\lfloor n^{1-\varepsilon}\right\rfloor} ; \hat{T}_{z}>\left\lfloor n^{1-\varepsilon}\right\rfloor, v_{n}^{\varepsilon}>\left\lfloor n^{1-\varepsilon}\right\rfloor\right)}_{=: J_{22}^{\prime \prime}} \\
& -\underbrace{\mathbb{E}_{x}\left(z+M_{\nu_{n}^{\varepsilon}} ; z+M_{v_{n}^{\varepsilon}}<0, \tau_{y}>v_{n}^{\varepsilon}, v_{n}^{\varepsilon} \leq\left\lfloor n^{1-\varepsilon}\right\rfloor\right)}_{=: J_{22}^{\prime}} .
\end{aligned}
$$

In the same way as in the proof of the bound of $J_{21}^{\prime \prime}$, replacing $v_{n}$ by $v_{n}^{\varepsilon}$, one can prove that

$$
-J_{22}^{\prime \prime} \leq c_{\varepsilon} \mathrm{e}^{-c_{\varepsilon} n^{\varepsilon}}(1+N(x)) .
$$

Moreover, using (5.1), on the event $\left\{\tau_{y}>v_{n}^{\varepsilon}\right\}$, we have $-\left(z+M_{v_{n}^{\varepsilon}}\right)<-r\left(X_{v_{n}^{\varepsilon}}\right)$. So, by Lemma 5.1 and the Markov property

$$
\begin{aligned}
J_{22}^{\prime} & \leq \mathbb{E}_{x}\left(\left|r\left(X_{v_{n}^{\varepsilon}}\right)\right| ; \hat{T}_{z}>v_{n}^{\varepsilon}, v_{n}^{\varepsilon} \leq\left\lfloor n^{1-\varepsilon}\right\rfloor\right) \\
& \leq \mathbb{E}_{x}\left(c\left(1+N\left(X_{v_{n}^{\varepsilon}}\right)\right) ; \hat{T}_{z}>v_{n}, v_{n} \leq\left\lfloor n^{1-\varepsilon}\right\rfloor\right) \\
& =c \int_{\mathbb{X}} \mathbb{E}_{x^{\prime}}\left(1+N\left(X_{\left\lfloor n^{\varepsilon}\right\rfloor}\right)\right) \mathbb{P}_{x}\left(X_{v_{n}} \in \mathrm{d} x^{\prime}, \hat{T}_{z}>v_{n}, v_{n} \leq\left\lfloor n^{1-\varepsilon}\right\rfloor\right) .
\end{aligned}
$$

Using (2.2),

$$
J_{22}^{\prime} \leq c \mathbb{E}_{x}\left(1+\mathrm{e}^{-c n^{\varepsilon}} N\left(X_{v_{n}}\right) ; \hat{T}_{z}>v_{n}, v_{n} \leq\left\lfloor n^{1-\varepsilon}\right\rfloor\right) .
$$

Therefore, from (6.17) with the notation $J_{21}^{\prime}$ from (6.18),

$$
J_{22}^{\prime} \leq J_{21}^{\prime}+c_{\varepsilon} \mathrm{e}^{-c_{\varepsilon} n^{\varepsilon}}(1+N(x)) .
$$

With (6.21), (6.23) and (6.24) we obtain

$$
J_{22} \leq\left(1+\frac{c_{\varepsilon}}{n^{\varepsilon}}\right) u_{\left\lfloor n^{1-\varepsilon}\right\rfloor}+c_{\varepsilon} \mathrm{e}^{-c_{\varepsilon} n^{\varepsilon}}(1+N(x)) .
$$


Inserting (6.26) and (6.22) into (6.16),

$$
J_{2} \leq\left(1+\frac{c_{\varepsilon}}{n^{\varepsilon}}\right) u_{\left\lfloor n^{1-\varepsilon}\right\rfloor}+c_{\varepsilon} \mathrm{e}^{-c_{\varepsilon} n^{\varepsilon}}(1+N(x)) .
$$

Now, inserting (6.15) and (6.27) into (6.14), we find (6.13).

COROllary 6.5. There exists $\varepsilon_{0}>0$ such that, for any $\varepsilon \in\left(0, \varepsilon_{0}\right), x \in \mathbb{X}$, $y \in \mathbb{R}, n \geq 2$ and any integer $k_{0} \in\{2, \ldots, n\}$,

$$
\mathbb{E}_{x}\left(y+S_{n} ; \tau_{y}>n\right) \leq\left(1+\frac{c_{\varepsilon}}{k_{0}^{\varepsilon}}\right)(\max (y, 0)+c N(x))+c_{\varepsilon} k_{0}^{1 / 2} .
$$

PROOF. First, using the definition of $\hat{T}_{z}$ and Lemma 6.4, with $z=y+r(x)$,

$$
\mathbb{E}_{x}\left(z+M_{n} ; \tau_{y}>n\right)=\mathbb{E}_{x}\left(z+M_{n} ; \hat{T}_{z}>n\right)-\mathbb{E}_{x}\left(z+M_{n} ; \tau_{y} \leq n, \hat{T}_{z}>n\right)
$$

$$
\begin{aligned}
& \leq \mathbb{E}_{x}\left(z+M_{n} ; \hat{T}_{z}>n\right) \\
& \leq\left(1+\frac{c_{\varepsilon}}{k_{0}^{\varepsilon}}\right)(\max (z, 0)+c N(x))+c_{\varepsilon} k_{0}^{1 / 2} .
\end{aligned}
$$

Now, using (5.1), Lemma 5.1 and (2.2),

$$
\begin{aligned}
\mathbb{E}_{x}\left(y+S_{n} ; \tau_{y}>n\right) & =\mathbb{E}_{x}\left(z+M_{n} ; \tau_{y}>n\right)-\mathbb{E}_{x}\left(r\left(X_{n}\right) ; \tau_{y}>n\right) \\
& \leq \mathbb{E}_{x}\left(z+M_{n} ; \tau_{y}>n\right)+c\left(1+\mathrm{e}^{-c n} N(x)\right) \\
& \leq\left(1+\frac{c_{\varepsilon}}{k_{0}^{\varepsilon}}\right)(\max (z, 0)+c N(x))+c_{\varepsilon} k_{0}^{1 / 2} .
\end{aligned}
$$

Using the definition of $z$ concludes the proof.

7. Existence and properties of the harmonic function. The idea is very simple. Set for brevity $V_{n}(x, y):=\mathbb{E}_{x}\left(y+S_{n} ; \tau_{y}>n\right)$. By the Markov property, $V_{n+1}(x, y)=\mathbf{Q}_{+} V_{n}(x, y)$. We show that $\lim _{n \rightarrow \infty} V_{n}(x, y)$ exists and is equal to $V(x, y):=-\mathbb{E}_{x}\left(M_{\tau_{y}}\right)$. Then the harmonicity of $V$ follows by the Lebesgue dominated convergence theorem. The key point of the proof is the integrability of the random variable $M_{\tau_{y}}$. To justify the applicability of the Lebesgue dominated convergence theorem we use Lemma 6.4. We also shall establish some properties of $V$. They will be deduced from those of the following two functions: $W(x, z):=-\mathbb{E}_{x}\left(M_{T_{z}}\right)$ and $\hat{W}(x, z):=-\mathbb{E}_{x}\left(M_{\hat{T}_{z}}\right)$. The strict positivity of $V$ is technically more delicate and therefore is deferred to the next section.

LEMMA 7.1. Let $x \in \mathbb{X}, y \in \mathbb{R}$ and $z=y+r(x)$. The random variables $M_{\hat{T}_{z}}$, $M_{T_{z}}$ and $M_{\tau_{y}}$ are integrable and

$$
\max \left\{\mathbb{E}_{x}\left(\left|M_{\hat{T}_{z}}\right|\right), \mathbb{E}_{x}\left(\left|M_{T_{z}}\right|\right), \mathbb{E}_{x}\left(\left|M_{\tau_{y}}\right|\right)\right\} \leq c(1+|z|+N(x))<+\infty
$$


In particular, the following functions are well defined, for any $x \in \mathbb{X}, y \in \mathbb{R}$ and $z \in \mathbb{R}$,

$$
\begin{aligned}
& V(x, y):=-\mathbb{E}_{x}\left(M_{\tau_{y}}\right), \quad W(x, z):=-\mathbb{E}_{x}\left(M_{T_{z}}\right) \quad \text { and } \\
& \hat{W}(x, z):=-\mathbb{E}_{x}\left(M_{\hat{T}_{z}}\right) .
\end{aligned}
$$

PROOF. Let $n \geq 1$. The stopping times $\tau_{y} \wedge n, T_{z} \wedge n$ and $\hat{T}_{z} \wedge n$ are bounded and satisfy $\tau_{y} \wedge n \leq \hat{T}_{z} \wedge n$ and $T_{z} \wedge n \leq \hat{T}_{z} \wedge n$. Since $\left(\left|M_{n}\right|\right)_{n \geq 0}$ is a submartingale, we have

$$
\max \left\{\mathbb{E}_{x}\left(\left|M_{\tau_{y} \wedge n}\right|\right), \mathbb{E}_{x}\left(\left|M_{T_{z} \wedge n}\right|\right)\right\} \leq \mathbb{E}_{x}\left(\left|M_{\hat{T}_{z} \wedge n}\right|\right) .
$$

Using the optional stopping theorem,

$$
\begin{aligned}
\mathbb{E}_{x}\left(\left|M_{\hat{T}_{z} \wedge n}\right|\right) \leq & -\mathbb{E}_{x}\left(z+M_{\hat{T}_{z}} ; \hat{T}_{z} \leq n\right)+\mathbb{E}_{x}\left(\left|z+M_{n}\right| ; \tau_{y}>n\right) \\
& +\mathbb{E}_{x}\left(z+M_{n} ; \tau_{y} \leq n, \hat{T}_{z}>n\right)+|z| \\
= & -\mathbb{E}_{x}\left(z+M_{n} ; \hat{T}_{z} \leq n\right)-2 \mathbb{E}_{x}\left(z+M_{n} ; z+M_{n} \leq 0, \tau_{y}>n\right) \\
& +\mathbb{E}_{x}\left(z+M_{n} ; \tau_{y}>n\right)+\mathbb{E}_{x}\left(z+M_{n} ; \tau_{y} \leq n, \hat{T}_{z}>n\right)+|z| \\
= & -z+2 \mathbb{E}_{x}\left(z+M_{n} ; \hat{T}_{z}>n\right) \\
& -2 \mathbb{E}_{x}\left(z+M_{n} ; z+M_{n} \leq 0, \tau_{y}>n\right)+|z| .
\end{aligned}
$$

On the event $\left\{z+M_{n} \leq 0, \tau_{y}>n\right\}$, by (5.1), it holds $\left|z+M_{n}\right| \leq\left|r\left(X_{n}\right)\right|$. Therefore, by Lemma 5.1 and point 1 of Hypothesis M4, we have

$$
-2 \mathbb{E}_{x}\left(z+M_{n} ; z+M_{n} \leq 0, \tau_{y}>n\right) \leq c(1+N(x)) .
$$

Using Lemma 6.4,

$$
\mathbb{E}_{x}\left(\left|M_{\hat{T}_{z}}\right| ; \hat{T}_{z} \leq n\right) \leq \mathbb{E}_{x}\left(\left|M_{\hat{T}_{z} \wedge n}\right|\right) \leq c(1+|z|+N(x)) .
$$

By the Lebesgue monotone convergence theorem and the fact that $\hat{T}_{z}<+\infty$, we deduce that $M_{\hat{T}_{z}}$ is $\mathbb{P}_{x}$-integrable and

$$
\mathbb{E}_{x}\left(\left|M_{\hat{T}_{z}}\right|\right) \leq c(1+|z|+N(x)) .
$$

In the same manner, using (7.1), (7.2) and Lemmas 5.5 and 5.6, we conclude that $M_{\tau_{y}}$ and $M_{T_{z}}$ are $\mathbb{P}_{x}$-integrable and

$$
\max \left\{\mathbb{E}_{x}\left(\left|M_{\tau_{y}}\right|\right), \mathbb{E}_{x}\left(\left|M_{T_{z}}\right|\right)\right\} \leq c(1+|z|+N(x)) .
$$

The assertion of the lemma follows obviously from the last two inequalities. 
Proposition 7.2. 1. Let $x \in \mathbb{X}, y \in \mathbb{R}$ and $z=y+r(x)$. Then

$$
V(x, y)=\lim _{n \rightarrow+\infty} \mathbb{E}_{x}\left(z+M_{n} ; \tau_{y}>n\right)=\lim _{n \rightarrow+\infty} \mathbb{E}_{x}\left(y+S_{n} ; \tau_{y}>n\right)
$$

and

$$
\begin{aligned}
& W(x, z)=\lim _{n \rightarrow+\infty} \mathbb{E}_{x}\left(z+M_{n} ; T_{z}>n\right), \\
& \hat{W}(x, z)=\lim _{n \rightarrow+\infty} \mathbb{E}_{x}\left(z+M_{n} ; \hat{T}_{z}>n\right) .
\end{aligned}
$$

2. For any $x \in \mathbb{X}$, the functions $y \mapsto V(x, y), z \mapsto W(x, z)$ and $z \mapsto \hat{W}(x, z)$ are nondecreasing on $\mathbb{R}$.

3. There exists $\varepsilon_{0}>0$ such that, for any $\varepsilon \in\left(0, \varepsilon_{0}\right), x \in \mathbb{X}, z \in \mathbb{R}$ and any integer $k_{0} \geq 2$,

$$
\hat{W}(x, z) \leq\left(1+\frac{c_{\varepsilon}}{k_{0}^{\varepsilon}}\right)(\max (z, 0)+c N(x))+c_{\varepsilon} k_{0}^{1 / 2}
$$

and, for any $x \in \mathbb{X}, y \in \mathbb{R}$ and $z=y+r(x)$,

$$
0 \leq \min \{V(x, y), W(x, z)\} \leq \max \{V(x, y), W(x, z)\} \leq \hat{W}(x, y) .
$$

In particular, for any $x \in \mathbb{X}$ and $y \in \mathbb{R}$,

$$
0 \leq V(x, y) \leq c(1+\max (y, 0)+N(x)) .
$$

4. For any $x \in \mathbb{X}$ and $y \in \mathbb{R}$,

$$
V(x, y)=\mathbf{Q}_{+} V(x, y):=\mathbb{E}_{x}\left(V\left(X_{1}, y+S_{1}\right) ; \tau_{y}>1\right)
$$

and $\left(V\left(X_{n}, y+S_{n}\right) \mathbb{1}_{\left\{\tau_{y}>n\right\}}\right)_{n \geq 0}$ is a $\mathbb{P}_{x}$-martingale.

Proof. Claim 1. Let $v$ be any of the stopping times $\tau_{y}, T_{z}$, or $\hat{T}_{z}$. By the martingale property, for $n \geq 1$,

$$
\mathbb{E}_{x}\left(z+M_{n} ; v>n\right)=z \mathbb{P}_{x}(v>n)-\mathbb{E}_{x}\left(M_{v} ; v \leq n\right) .
$$

Using Lemmas 5.5, 5.6, 5.7, 7.1 and the Lebesgue dominated convergence theorem,

$$
\mathbb{E}_{x}\left(z+M_{n} ; v>n\right)=-\mathbb{E}_{x}\left(M_{v}\right)
$$

Moreover, by (5.1),

$$
\mathbb{E}_{x}\left(y+S_{n} ; \tau_{y}>n\right)=\mathbb{E}_{x}\left(z+M_{n} ; \tau_{y}>n\right)-\mathbb{E}_{x}\left(r\left(X_{n}\right) ; \tau_{y}>n\right) .
$$

Since, by Lemma 5.1, point 1 of Hypothesis M4 and Lemma 5.5, we have

$$
\begin{aligned}
\left|\mathbb{E}_{x}\left(r\left(X_{n}\right) ; \tau_{y}>n\right)\right| & \leq c \mathbb{E}_{x}^{1 / 2}\left(\left(1+N\left(X_{n}\right)\right)^{2}\right) \mathbb{P}_{x}^{1 / 2}\left(\tau_{y}>n\right) \\
& \leq c(1+N(x)) \mathbb{P}_{x}^{1 / 2}\left(\tau_{y}>n\right) \underset{n \rightarrow+\infty}{\longrightarrow} 0
\end{aligned}
$$

claim 1 follows. 
Proof of claim 2. Let $x \in \mathbb{X}$. For any $y^{\prime} \leq y$, we obviously have $\tau_{y^{\prime}} \leq \tau_{y}$. Therefore, for $n \geq 1$,

$$
\mathbb{E}_{x}\left(y^{\prime}+S_{n} ; \tau_{y^{\prime}}>n\right) \leq \mathbb{E}_{x}\left(y+S_{n} ; \tau_{y^{\prime}}>n\right) \leq \mathbb{E}_{x}\left(y+S_{n} ; \tau_{y}>n\right) .
$$

Taking the limit as $n \rightarrow+\infty$ and using claim 1, it follows that $V\left(x, y^{\prime}\right) \leq V(x, y)$. In the same way $W\left(x, z^{\prime}\right) \leq W(x, z)$ for $z^{\prime} \leq z$. To prove the monotonicity of $\hat{W}$, we note that, for any $z^{\prime} \leq z, y^{\prime}=z^{\prime}-r(x)$ and $y=z-r(x)$, we have $\hat{T}_{z^{\prime}}=$ $\min \left\{k \geq \tau_{y^{\prime}}: z^{\prime}+M_{k} \leq 0\right\} \leq \min \left\{k \geq \tau_{y}: z^{\prime}+M_{k} \leq 0\right\} \leq \hat{T}_{z}$. So

$$
\begin{aligned}
\mathbb{E}_{x}\left(z^{\prime}+M_{n} ; \hat{T}_{z^{\prime}}>n\right) \leq & \mathbb{E}_{x}\left(z+M_{n} ; \hat{T}_{z^{\prime}}>n, \hat{T}_{z}>n\right) \\
\leq & \mathbb{E}_{x}\left(y+S_{n} ; \tau_{y}>n\right)+\mathbb{E}_{x}\left(\left|r\left(X_{n}\right)\right| ; \tau_{y}>n\right) \\
& \quad+\mathbb{E}_{x}\left(z+M_{n} ; \tau_{y} \leq n, \hat{T}_{z}>n\right) \\
\leq & \mathbb{E}_{x}\left(z+M_{n} ; \hat{T}_{z}>n\right)+2 \mathbb{E}_{x}\left(\left|r\left(X_{n}\right)\right| ; \tau_{y}>n\right) .
\end{aligned}
$$

As in (7.6), taking the limit as $n \rightarrow+\infty$, by the claim 1 , we have $\hat{W}\left(x, z^{\prime}\right) \leq$ $\hat{W}(x, z)$.

Proof of claim 3. The inequality (7.3) is a direct consequence of claim 1 and Lemma 6.4. Moreover, taking the limit as $n \rightarrow \infty$ in (6.28), we get $V(x, y) \leq$ $\hat{W}(x, z)$.

To bound $W$, we write, for $n \geq 1$,

$$
\begin{aligned}
\mathbb{E}_{x}\left(z+M_{n} ; T_{z}>n\right)= & \mathbb{E}_{x}\left(z+M_{n} ; \tau_{y} \leq n, \hat{T}_{z}>n, T_{z}>n\right) \\
& +\mathbb{E}_{x}\left(z+M_{n} ; z+M_{n}>0, \tau_{y}>n, T_{z}>n\right) .
\end{aligned}
$$

Since $z+M_{n}>0$ on the event $\left\{\tau_{y} \leq n, \hat{T}_{z}>n\right\}$,

$$
\begin{aligned}
\mathbb{E}_{x}\left(z+M_{n} ; T_{z}>n\right) \leq & \mathbb{E}_{x}\left(z+M_{n} ; \tau_{y} \leq n, \hat{T}_{z}>n\right) \\
& +\mathbb{E}_{x}\left(z+M_{n} ; z+M_{n}>0, \tau_{y}>n\right) \\
= & \mathbb{E}_{x}\left(z+M_{n} ; \hat{T}_{z}>n\right) \\
& \quad-\mathbb{E}_{x}\left(z+M_{n} ; z+M_{n} \leq 0, \tau_{y}>n\right) .
\end{aligned}
$$

Using the approximation (5.1),

$$
\mathbb{E}_{x}\left(z+M_{n} ; T_{z}>n\right) \leq \mathbb{E}_{x}\left(z+M_{n} ; \hat{T}_{z}>n\right)+\mathbb{E}_{x}\left(\left|r\left(X_{n}\right)\right| ; \tau_{y}>n\right) .
$$

As in (7.6), using claim 1,

$$
W(x, z) \leq \hat{W}(x, z) .
$$

Now, since $y+S_{n}$ is positive on the event $\left\{\tau_{y}>n\right\}$, by the claim 1 , we see that $V(x, y) \geq 0$ and in the same way, $W(x, z) \geq 0$. This proves (7.4). 
Inequality (7.5) follows from (7.3) and (7.4).

Proof of claim 4. By the Markov property, for $n \geq 1$,

$$
\begin{aligned}
V_{n+1}(x, y) & :=\mathbb{E}_{x}\left(y+S_{n+1} ; \tau_{y}>n+1\right) \\
& =\int_{\mathbb{X} \times \mathbb{R}} V_{n}\left(x^{\prime}, y^{\prime}\right) \mathbb{P}_{x}\left(X_{1} \in \mathrm{d} x^{\prime}, y+S_{1} \in \mathrm{d} y^{\prime}, \tau_{y}>1\right),
\end{aligned}
$$

where, by Corollary 6.5, $V_{n}\left(x^{\prime}, y^{\prime}\right) \leq c\left(1+\left|y^{\prime}\right|+N\left(x^{\prime}\right)\right)$ and by point 1 of Hypothesis M4,

$$
\mathbb{E}_{x}\left(1+\left|y+S_{1}\right|+N\left(X_{1}\right)\right) \leq c(1+|y|+N(x))<+\infty .
$$

Taking the limit in (7.8), by the Lebesgue dominated convergence theorem, we have

$$
V(x, y)=\mathbf{Q}_{+} V(x, y):=\mathbb{E}_{x}\left(V\left(X_{1}, y+S_{1}\right) ; \tau_{y}>1\right) .
$$

8. Positivity of the harmonic function. The aim of this section is to prove that the harmonic function $V$ is nonidentically zero and to precise its support.

For any $x \in \mathbb{X}, z \in \mathbb{R}$ and $n \geq 0$, denote for brevity,

$$
\hat{W}_{n}(x, z)=\hat{W}\left(X_{n}, z+M_{n}\right) \mathbb{1}_{\left\{\hat{T}_{z}>n\right\}} .
$$

Although it is easy to verify that $\hat{W}(x, z) \geq z$ (see Lemma 8.1) which, in turn, ensures that $\hat{W}(x, z)>0$ for any $z>0$, it is not straightforward to give a lower bound for the function $V$. We show that $V(x, y)=\lim _{n \rightarrow+\infty} \mathbb{E}_{x}\left(\hat{W}_{n}(x, z)\right.$; $\left.\tau_{y}>n\right)$ (Lemma 8.2) and use the fact that $\left(\hat{W}_{n}(x, z) \mathbb{1}_{\left\{\tau_{y}>n\right\}}\right)_{n \geq 0}$ is a $\mathbb{P}_{x^{-}}$ supermartingale (Lemma 8.1). By a recurrent procedure similar to that used in Lemma 6.4, we obtain a lower bound for $V$ (Lemma 8.6) which subsequently is used to prove the positivity of $V$ (Lemma 8.8).

LEMMA 8.1. 1. For any $x \in \mathbb{X}$ and $z \in \mathbb{R}$,

$$
\hat{W}(x, z) \geq z \text {. }
$$

2. For any $x \in \mathbb{X}$,

$$
\lim _{z \rightarrow+\infty} \frac{\hat{W}(x, z)}{z}=1 .
$$

3. The function $\hat{W}$ is subharmonic, that is, for any $x \in \mathbb{X}, z \in \mathbb{R}$ and $n \geq 0$,

$$
\mathbb{E}_{x}\left(\hat{W}_{n}(x, z)\right) \geq \hat{W}(x, z) .
$$

4. For any $x \in \mathbb{X}$ and $z \in \mathbb{R},\left(\hat{W}_{n}(x, z) \mathbb{1}_{\left\{\tau_{y}>n\right\}}\right)_{n \geq 0}$ is a $\mathbb{P}_{x}$-supermartingale. 
Proof. Claim 1. By the Doob optional theorem and the definition of $\hat{T}_{z}$, for any $n \geq 1$,

$$
\mathbb{E}_{x}\left(z+M_{n} ; \hat{T}_{z}>n\right)=z-\mathbb{E}_{x}\left(z+M_{\hat{T}_{z}} ; \hat{T}_{z} \leq n\right) \geq z .
$$

Taking the limit as $n \rightarrow+\infty$ and using point 1 of Proposition 7.2 proves claim 1 .

Proof of claim 2. By claim $1, \liminf _{z \rightarrow+\infty} \hat{W}(x, z) / z \geq 1$. Moreover, by (7.3), for any $k_{0} \geq 2$,

$$
\limsup _{z \rightarrow \infty} \frac{\hat{W}(x, z)}{z} \leq\left(1+\frac{c_{\varepsilon}}{k_{0}^{\varepsilon}}\right) .
$$

Taking the limit as $k_{0} \rightarrow+\infty$, the claim follows.

Proof of claim 3. Recall the notation $y=z-r(x)$. Using the Markov property, as in the proof of Lemma 5.3, for any $k \geq 1$,

$$
\begin{aligned}
\mathbb{E}_{x}(z+ & \left.M_{n+k} ; \hat{T}_{z}>n+k\right) \\
= & \int_{\mathbb{X} \times \mathbb{R}} \mathbb{E}_{x^{\prime}}\left(z^{\prime}+M_{n} ; \hat{T}_{z^{\prime}}>n\right) \mathbb{P}_{x}\left(X_{k} \in \mathrm{d} x^{\prime}, z+M_{k} \in \mathrm{d} z^{\prime}, \tau_{y}>k\right) \\
& +\int_{\mathbb{X} \times \mathbb{R}} \mathbb{E}_{x^{\prime}}\left(z^{\prime}+M_{n} ; T_{z^{\prime}}>n\right) \\
& \times \mathbb{P}_{x}\left(X_{k} \in \mathrm{d} x^{\prime}, z+M_{k} \in \mathrm{d} z^{\prime}, \tau_{y} \leq k, \hat{T}_{z}>k\right) .
\end{aligned}
$$

We shall find the limits as $n \rightarrow+\infty$ of the two terms in the right-hand side. By Lemmas 6.4 and 5.1, $\mathbb{E}_{x^{\prime}}\left(z^{\prime}+M_{n} ; \hat{T}_{z^{\prime}}>n\right) \leq c\left(1+\left|y^{\prime}\right|+N\left(x^{\prime}\right)\right)$, with $y^{\prime}=z^{\prime}-$ $r\left(x^{\prime}\right)$. Moreover, by point 1 of Hypothesis M4, $\mathbb{E}_{x}\left(1+\left|y+S_{k}\right|+N\left(X_{k}\right)\right) \leq c k(1+$ $|y|+N(x))<+\infty$. So, by the Lebesgue dominated convergence theorem and point 1 of Proposition 7.2,

$$
\begin{gathered}
\int_{\mathbb{X} \times \mathbb{R}} \mathbb{E}_{x^{\prime}}\left(z^{\prime}+M_{n} ; \hat{T}_{z^{\prime}}>n\right) \mathbb{P}_{x}\left(X_{k} \in \mathrm{d} x^{\prime}, z+M_{k} \in \mathrm{d} z^{\prime}, \tau_{y}>k\right) \\
\underset{n \rightarrow+\infty}{\longrightarrow} \mathbb{E}_{x}\left(\hat{W}\left(X_{k}, z+M_{k}\right) ; \tau_{y}>k\right) .
\end{gathered}
$$

Moreover, using (7.7), Lemmas 6.4 and 5.1 and point 1 of Hypothesis M4,

$$
\mathbb{E}_{x^{\prime}}\left(z^{\prime}+M_{n} ; T_{z^{\prime}}>n\right) \leq c\left(1+\left|z^{\prime}\right|+N\left(x^{\prime}\right)\right) .
$$

Again, by the Lebesgue dominated convergence theorem and point 1 of Proposition 7.2, we have

$$
\begin{aligned}
& \int_{\mathbb{X} \times \mathbb{R}} \mathbb{E}_{X^{\prime}}\left(z^{\prime}+M_{n} ; T_{z^{\prime}}>n\right) \mathbb{P}_{x}\left(X_{k} \in \mathrm{d} x^{\prime}, z+M_{k} \in \mathrm{d} z^{\prime}, \tau_{y} \leq k, \hat{T}_{z}>k\right) \\
& \underset{n \rightarrow+\infty}{\longrightarrow} \mathbb{E}_{x}\left(W\left(X_{k}, z+M_{k}\right) ; \tau_{y} \leq k, \hat{T}_{z}>k\right) .
\end{aligned}
$$


Putting together (8.2), (8.3), (8.4) and using point 1 of Proposition 7.2,

$$
\begin{aligned}
\hat{W}(x, z)= & \mathbb{E}_{x}\left(\hat{W}\left(X_{k}, z+M_{k}\right) ; \tau_{y}>k\right) \\
& +\mathbb{E}_{x}\left(W\left(X_{k}, z+M_{k}\right) ; \tau_{y} \leq k, \hat{T}_{z}>k\right) .
\end{aligned}
$$

Now, taking into account (7.4) and the identity $\left\{\tau_{y}>k\right\}=\left\{\tau_{y}>k, \hat{T}_{z}>k\right\}$, we obtain claim 3 .

Proof of claim 4. By point 3 of Proposition 7.2, $W$ is a nonnegative function. Therefore, using (8.5),

$$
\hat{W}(x, z) \geq \mathbb{E}_{x}\left(\hat{W}\left(X_{1}, z+M_{1}\right) ; \tau_{y}>1\right),
$$

which implies that $\left(\hat{W}_{n}(x, z) \mathbb{1}_{\left\{\tau_{y}>n\right\}}\right)_{n \geq 0}$ is a supermartingale.

LEMMA 8.2. For any $x \in \mathbb{X}, y \in \mathbb{R}$ and $z=y+r(x)$,

$$
V(x, y)=\lim _{n \rightarrow+\infty} \mathbb{E}_{x}\left(\hat{W}_{n}(x, z) ; \tau_{y}>n\right) .
$$

PROOF. For any $n \geq 1, x \in \mathbb{X}, y \in \mathbb{R}$ and $z=y+r(x)$,

$$
\mathbb{E}_{x}\left(z+M_{n} ; \tau_{y}>n\right)=\mathbb{E}_{x}\left(z+M_{n} ; \hat{T}_{z}>n\right)-\mathbb{E}_{x}\left(z+M_{n} ; \tau_{y} \leq n, \hat{T}_{z}>n\right) .
$$

By point 1 of Lemma 8.1 , on the event $\left\{\hat{T}_{z}>n\right\}$, we have $z+M_{n} \leq \hat{W}_{n}(x, z)$ and therefore

$$
\begin{gathered}
\mathbb{E}_{x}\left(z+M_{n} ; \tau_{y}>n\right) \geq \mathbb{E}_{x}\left(z+M_{n} ; \hat{T}_{z}>n\right)-\mathbb{E}_{x}\left(\hat{W}_{n}(x, z)\right) \\
+\mathbb{E}_{x}\left(\hat{W}_{n}(x, z) ; \tau_{y}>n\right) .
\end{gathered}
$$

Moreover, by (7.3), for any $\delta>0$,

$$
\begin{aligned}
\mathbb{E}_{x}\left(\hat{W}_{n}(x, z)\right) \leq & (1+\delta) \mathbb{E}_{x}\left(z+M_{n} ; \hat{T}_{z}>n\right)+c_{\delta} \mathbb{E}_{x}\left(1+N\left(X_{n}\right) ; \hat{T}_{z}>n\right) \\
& -(1+\delta) \mathbb{E}_{x}\left(z+M_{n} ; z+M_{n}<0, \tau_{y}>n\right) .
\end{aligned}
$$

On the event $\left\{z+M_{n}<0, \tau_{y}>n\right\}$, by (5.1), it holds $r\left(X_{n}\right)<z+M_{n}<0$. Therefore, using Lemma 5.1,

$$
\mathbb{E}_{x}\left(\hat{W}_{n}(x, z)\right) \leq(1+\delta) \mathbb{E}_{x}\left(z+M_{n} ; \hat{T}_{z}>n\right)+c_{\delta} \mathbb{E}_{x}\left(1+N\left(X_{n}\right) ; \hat{T}_{z}>n\right) .
$$

By the Markov property and (2.2),

$$
\begin{aligned}
\mathbb{E}_{x}\left(1+N\left(X_{n}\right) ; \hat{T}_{z}>n\right) & \leq c \mathbb{E}_{x}\left(1+\mathrm{e}^{-c n / 2} N\left(X_{\lfloor n / 2\rfloor}\right) ; \hat{T}_{z}>\lfloor n / 2\rfloor\right) \\
& \leq c \mathbb{P}_{x}\left(\hat{T}_{z}>\lfloor n / 2\rfloor\right)+c \mathrm{e}^{-c n}(1+N(x)) .
\end{aligned}
$$

By Lemma 5.7 and point 1 of Proposition 7.2,

$$
\lim _{n \rightarrow+\infty} \mathbb{E}_{x}\left(\hat{W}_{n}(x, z)\right) \leq(1+\delta) \hat{W}(x, z) .
$$


Taking the limit as $n \rightarrow+\infty$ in (8.6) and using the previous bound, we obtain that

$$
V(x, y) \geq-\delta \hat{W}(x, z)+\lim _{n \rightarrow+\infty} \mathbb{E}_{x}\left(\hat{W}_{n}(x, z) ; \tau_{y}>n\right) .
$$

Since this inequality holds true for any $\delta>0$ small enough, we obtain the bound

$$
\lim _{n \rightarrow+\infty} \mathbb{E}_{x}\left(\hat{W}_{n}(x, z) ; \tau_{y}>n\right) \leq V(x, y) .
$$

Now, by point 1 of Lemma 8.1,

$$
\mathbb{E}_{x}\left(z+M_{n} ; \tau_{y}>n\right) \leq \mathbb{E}_{x}\left(\hat{W}\left(X_{n}, z+M_{n}\right) ; \tau_{y}>n\right) .
$$

Taking the limit as $n \rightarrow+\infty$ and using point 1 of Proposition 7.2, we obtain that

$$
V(x, y) \leq \lim _{n \rightarrow+\infty} \mathbb{E}_{x}\left(\hat{W}_{n}(x, z) ; \tau_{y}>n\right) .
$$

Together with (8.8), this concludes the proof.

REMARK 8.3. Taking the limit in point 3 of Lemma 8.1,

$$
\lim _{n \rightarrow+\infty} \mathbb{E}_{x}\left(\hat{W}_{n}(x, z)\right) \geq \hat{W}(x, z) .
$$

Coupling this result with (8.7), it follows that

$$
\lim _{n \rightarrow+\infty} \mathbb{E}_{x}\left(\hat{W}_{n}(x, z)\right)=\hat{W}(x, z) .
$$

LEMMA 8.4. There exists $\varepsilon_{0}>0$ such that, for any $\varepsilon \in\left(0, \varepsilon_{0}\right), n \geq 1, x \in \mathbb{X}$, $z \in \mathbb{R}$ and $y=z-r(x)$, we have

$$
\mathbb{E}_{x}\left(\hat{W}_{n}(x, z) ; \tau_{y}>n\right) \geq \hat{W}(x, z)+c \min (z, 0)-c_{\varepsilon}\left(n^{1 / 2-2 \varepsilon}+n^{2 \varepsilon} N(x)\right) .
$$

PROOF. Using point 3 of Lemma 8.1, the bound (7.3) and point 1 of Hypothesis M4, we have, for any $n \geq 1$,

$$
\begin{aligned}
\mathbb{E}_{x}\left(\hat{W}_{n}(x, z) ; \tau_{y}>n\right) & =\mathbb{E}_{x}\left(\hat{W}_{n}(x, z)\right)-\mathbb{E}_{x}\left(\hat{W}_{n}(x, z) ; \tau_{y} \leq n\right) \\
& \geq \hat{W}(x, z)-c \mathbb{E}_{x}\left(z+M_{n} ; \tau_{y} \leq n, \hat{T}_{z}>n\right)-c(1+N(x)) .
\end{aligned}
$$

Again by point 1 of M4, Lemma 6.2 and the Doob optional stopping theorem,

$$
\begin{aligned}
\mathbb{E}_{x}\left(\hat{W}_{n}(x, z) ; \tau_{y}>n\right) \geq & \hat{W}(x, z)-c\left[\mathbb{E}_{x}\left(z+M_{n} ; \hat{T}_{z}>n\right)-\mathbb{E}_{x}\left(z+M_{n} ; \tau_{y}>n\right)\right] \\
& -c(1+N(x)) \\
\geq & \hat{W}(x, z)-c\left[\max (z, 0)-z+\mathbb{E}_{x}\left(z+M_{\tau_{y}} ; \tau_{y} \leq n\right)\right] \\
& -c_{\varepsilon}\left(n^{1 / 2-2 \varepsilon}+n^{2 \varepsilon} N(x)\right)-c(1+N(x)) .
\end{aligned}
$$


By (5.1), $z+M_{\tau_{y}} \leq r\left(X_{\tau_{y}}\right)$. Therefore, in the same way as in the proof of (6.2),

$$
\mathbb{E}_{x}\left(z+M_{\tau_{y}} ; \tau_{y} \leq n\right) \leq c \mathbb{E}_{x}\left(1+N\left(X_{\tau_{y}}\right) ; \tau_{y} \leq n\right) \leq c_{\varepsilon} n^{1 / 2-2 \varepsilon}+c_{\varepsilon} N(x) .
$$

Together with the previous bound, this implies that

$$
\mathbb{E}_{x}\left(\hat{W}_{n}(x, z) ; \tau_{y}>n\right) \geq \hat{W}(x, z)+c \min (z, 0)-c_{\varepsilon}\left(n^{1 / 2-2 \varepsilon}+n^{2 \varepsilon} N(x)\right) .
$$

LEMMA 8.5. There exists $\varepsilon_{0}>0$ such that, for any $\varepsilon \in\left(0, \varepsilon_{0}\right), n \geq 2, k_{0} \in$ $\{2, \ldots, n\}, x \in \mathbb{X}$ and $z \in \mathbb{R}$, with $y=z-r(x)$, we have

$$
\mathbb{E}_{x}\left(\hat{W}_{n}(x, z) ; \tau_{y}>n\right) \geq \mathbb{E}_{x}\left(\hat{W}_{k_{0}}(x, z) ; \tau_{y}>k_{0}\right)-\frac{c_{\varepsilon}}{k_{0}^{\varepsilon}}(\max (z, 0)+1+N(x))
$$

PROOF. Let $\varepsilon \in(0,1)$. Set for brevity $u_{n}:=\mathbb{E}_{x}\left(\hat{W}_{n}(x, z) ; \tau_{y}>n\right)$ for $n \geq 1$. By point 4 of Lemma 8.1 , the sequence $\left(u_{n}\right)_{n \geq 1}$ is nonincreasing. We shall prove that

$$
u_{n} \geq u_{\left\lfloor n^{1-\varepsilon}\right\rfloor}-\frac{c_{\varepsilon}}{n^{\varepsilon}}(\max (z, 0)+1+N(x)) .
$$

By Lemma 9.2 of [18] on the convergence of recursively bounded nonincreasing sequences, we conclude that, for any $n \geq 2$ and $k_{0} \in\{2, \ldots, n\}$,

$$
u_{n} \geq u_{k_{0}}-\frac{c_{\varepsilon}}{k_{0}^{\varepsilon}}(\max (z, 0)+1+N(x)),
$$

which proves the assertion of the lemma.

It remains to establish (8.9). Consider the stopping time $v_{n}^{\varepsilon}=v_{n}+\left\lfloor n^{\varepsilon}\right\rfloor$. By the Markov property, with $y^{\prime}=z^{\prime}-r\left(x^{\prime}\right)$,

$$
\begin{aligned}
u_{n} \geq & \mathbb{E}_{x}\left(\hat{W}_{n}(x, z) ; \tau_{y}>n, v_{n}^{\varepsilon} \leq\left\lfloor n^{1-\varepsilon}\right\rfloor\right) \\
= & \sum_{k=\left\lfloor n^{\varepsilon}\right\rfloor+1}^{\left\lfloor n^{1-\varepsilon}\right\rfloor} \int_{\mathbb{X} \times \mathbb{R}} \mathbb{E}_{x^{\prime}}\left(\hat{W}_{n-k}\left(x^{\prime}, z^{\prime}\right) ; \tau_{y^{\prime}}>n-k\right) \\
& \times \mathbb{P}_{x}\left(X_{k} \in \mathrm{d} x^{\prime}, z+M_{k} \in \mathrm{d} z^{\prime}, \tau_{y}>k, v_{n}^{\varepsilon}=k\right) .
\end{aligned}
$$

Using Lemma 8.4, we obtain

$$
\begin{aligned}
u_{n} \geq & \mathbb{E}_{x}\left(\hat{W}_{v_{n}^{\varepsilon}}(x, z) ; \tau_{y}>v_{n}^{\varepsilon}, v_{n}^{\varepsilon} \leq\left\lfloor n^{1-\varepsilon}\right\rfloor\right) \\
& +c \mathbb{E}_{x}\left(\min \left(z+M_{v_{n}^{\varepsilon}}, 0\right) ; \tau_{y}>v_{n}^{\varepsilon}, v_{n}^{\varepsilon} \leq\left\lfloor n^{1-\varepsilon}\right\rfloor\right) \\
& -c_{\varepsilon} \mathbb{E}_{x}\left(n^{1 / 2-2 \varepsilon}+n^{2 \varepsilon} N\left(X_{v_{n}^{\varepsilon}}\right) ; \tau_{y}>v_{n}^{\varepsilon}, v_{n}^{\varepsilon} \leq\left\lfloor n^{1-\varepsilon}\right\rfloor\right) .
\end{aligned}
$$

On the event $\left\{z+M_{v_{n}^{\varepsilon}} \leq 0, \tau_{y}>v_{n}^{\varepsilon}\right\}$, by (5.1), we have $0 \geq z+M_{v_{n}^{\varepsilon}} \geq r\left(X_{v_{n}^{\varepsilon}}\right)$. Therefore, by Lemma 5.1,

$$
\begin{aligned}
& \mathbb{E}_{x}\left(\min \left(z+M_{v_{n}^{\varepsilon}}, 0\right) ; \tau_{y}>v_{n}^{\varepsilon}, v_{n}^{\varepsilon} \leq\left\lfloor n^{1-\varepsilon}\right\rfloor\right) \\
& \quad \geq-c \mathbb{E}_{x}\left(1+N\left(X_{v_{n}^{\varepsilon}}\right) ; \tau_{y}>v_{n}^{\varepsilon}, v_{n}^{\varepsilon} \leq\left\lfloor n^{1-\varepsilon}\right\rfloor\right) .
\end{aligned}
$$


Consequently, using point 4 of Lemma 8.1 and (2.2),

$$
\begin{aligned}
u_{n} \geq & \mathbb{E}_{x}\left(\hat{W}_{\left\lfloor n^{1-\varepsilon}\right\rfloor}(x, z) ; \tau_{y}>\left\lfloor n^{1-\varepsilon}\right\rfloor, v_{n}^{\varepsilon} \leq\left\lfloor n^{1-\varepsilon}\right\rfloor\right) \\
& \quad-c_{\varepsilon} \mathbb{E}_{x}\left(n^{1 / 2-2 \varepsilon}+\mathrm{e}^{-c_{\varepsilon} n^{\varepsilon}} N\left(X_{v_{n}}\right) ; \tau_{y}>v_{n}, v_{n} \leq\left\lfloor n^{1-\varepsilon}\right\rfloor\right) .
\end{aligned}
$$

By the definition of $v_{n}$, we have $n^{1 / 2-2 \varepsilon} \leq\left(z+M_{v_{n}}\right) / n^{\varepsilon}$. Then as in (6.17),

$$
\begin{aligned}
u_{n} \geq & \mathbb{E}_{x}\left(\hat{W}_{\left\lfloor n^{1-\varepsilon}\right\rfloor}(x, z) ; \tau_{y}>\left\lfloor n^{1-\varepsilon}\right\rfloor, v_{n}^{\varepsilon} \leq\left\lfloor n^{1-\varepsilon}\right\rfloor\right) \\
& -\frac{c_{\varepsilon}}{n^{\varepsilon}} \mathbb{E}_{x}\left(z+M_{v_{n}} ; \tau_{y}>v_{n}, v_{n} \leq\left\lfloor n^{1-\varepsilon}\right\rfloor\right) \\
& -c_{\varepsilon} \mathrm{e}^{-c_{\varepsilon} n^{\varepsilon}}(1+N(x)) .
\end{aligned}
$$

Rearranging the terms, we have

$$
\begin{aligned}
u_{n} \geq & u_{\left\lfloor n^{1-\varepsilon}\right\rfloor}-c_{\varepsilon} \mathrm{e}^{-c_{\varepsilon} n^{\varepsilon}}(1+N(x)) \\
& -\frac{c_{\varepsilon}}{n^{\varepsilon}} \underbrace{\mathbb{E}_{x}\left(z+M_{v_{n}} ; \tau_{y}>v_{n}, v_{n} \leq\left\lfloor n^{1-\varepsilon}\right\rfloor\right)}_{=: I_{1}} \\
& -\underbrace{\mathbb{E}_{x}\left(\hat{W}_{\left\lfloor n^{1-\varepsilon}\right\rfloor}(x, z) ; \tau_{y}>\left\lfloor n^{1-\varepsilon}\right\rfloor, v_{n}^{\varepsilon}>\left\lfloor n^{1-\varepsilon}\right\rfloor\right)}_{=: I_{2}} .
\end{aligned}
$$

Bound of $I_{1}$. To bound $I_{1}$ we use the facts that, by the definition of $v_{n}, z+M_{v_{n}}>$ $n^{1 / 2-\varepsilon}>0$ and that $\hat{T}_{z} \geq \tau_{y}$. Taking into account Lemma 5.4, we have

$$
\begin{aligned}
I_{1} & \leq \mathbb{E}_{x}\left(z+M_{\left\lfloor n^{1-\varepsilon}\right\rfloor} ; \hat{T}_{z}>\left\lfloor n^{1-\varepsilon}\right\rfloor, v_{n} \leq\left\lfloor n^{1-\varepsilon}\right\rfloor\right) \\
& =\mathbb{E}_{x}\left(z+M_{\left\lfloor n^{1-\varepsilon}\right\rfloor} ; \hat{T}_{z}>\left\lfloor n^{1-\varepsilon}\right\rfloor\right)-J_{21}^{\prime \prime},
\end{aligned}
$$

where $J_{21}^{\prime \prime}$ is defined in (6.19). Now, it follows from Lemma 5.4 and point 1 of Proposition 7.2, that $\left(\mathbb{E}_{x}\left(z+M_{\left\lfloor n^{1-\varepsilon}\right\rfloor} ; \hat{T}_{z}>\left\lfloor n^{1-\varepsilon}\right\rfloor\right)\right)_{n \geq 0}$ is a nondecreasing sequence which converges to $\hat{W}(x, z)$ and so $\mathbb{E}_{x}\left(z+M_{\left\lfloor n^{1-\varepsilon}\right\rfloor} ; \hat{T}_{z}>\left\lfloor n^{1-\varepsilon}\right\rfloor\right) \leq$ $\hat{W}(x, z)$. Using (6.20), we find that

$$
I_{1} \leq \hat{W}(x, z)+c_{\varepsilon} \mathrm{e}^{-c_{\varepsilon} n^{\varepsilon}}(1+N(x)) .
$$

Bound of $I_{2}$. By (8.1) and (7.3),

$$
\begin{aligned}
I_{2} \leq & \left.c \mathbb{E}_{x}\left(z+M_{\left\lfloor n^{1-\varepsilon}\right\rfloor}\left(1-\mathbb{1}_{\left\{z+M_{\lfloor n} 1-\varepsilon\right\rfloor}<0\right\}\right) ; \hat{T}_{z}>\left\lfloor n^{1-\varepsilon}\right\rfloor, v_{n}^{\varepsilon}>\left\lfloor n^{1-\varepsilon}\right\rfloor\right) \\
& +c \mathbb{E}_{x}\left(1+N\left(X_{\left\lfloor n^{1-\varepsilon}\right\rfloor}\right) ; \hat{T}_{z}>\left\lfloor n^{1-\varepsilon}\right\rfloor, v_{n}^{\varepsilon}>\left\lfloor n^{1-\varepsilon}\right\rfloor\right) .
\end{aligned}
$$

On the event $\left\{z+M_{\left\lfloor n^{1-\varepsilon}\right\rfloor}<0, \hat{T}_{z}>\left\lfloor n^{1-\varepsilon}\right\rfloor\right\}=\left\{z+M_{\left\lfloor n^{1-\varepsilon}\right\rfloor}<0, \tau_{y}>\left\lfloor n^{1-\varepsilon}\right\rfloor\right\}$, it holds $z+M_{\left\lfloor n^{1-\varepsilon}\right\rfloor}>r\left(X_{\left\lfloor n^{1-\varepsilon}\right\rfloor}\right)$. Therefore, using Lemma 5.1,

$$
I_{2} \leq c \mathbb{E}_{x}\left(z+M_{\left\lfloor n^{1-\varepsilon}\right\rfloor}+1+N\left(X_{\left\lfloor n^{1-\varepsilon}\right\rfloor}\right) ; \hat{T}_{z}>\left\lfloor n^{1-\varepsilon}\right\rfloor, v_{n}^{\varepsilon}>\left\lfloor n^{1-\varepsilon}\right\rfloor\right) .
$$


By Lemma 5.4,

$$
\mathbb{E}_{x}\left(z+M_{\left\lfloor n^{1-\varepsilon}\right\rfloor} ; \hat{T}_{z}>\left\lfloor n^{1-\varepsilon}\right\rfloor, v_{n}^{\varepsilon}>\left\lfloor n^{1-\varepsilon}\right\rfloor\right) \leq J_{1},
$$

where $J_{1}$ is defined in (6.14). Using inequalities (6.15), (2.2) and Lemma 6.3, with $m_{\varepsilon}=\left\lfloor n^{1-\varepsilon}\right\rfloor-\left\lfloor n^{\varepsilon}\right\rfloor$, we obtain

$$
\begin{aligned}
I_{2} & \leq c_{\varepsilon} \mathrm{e}^{-c_{\varepsilon} n^{\varepsilon}}(1+N(x))+c \mathbb{E}_{x}\left(1+\mathrm{e}^{-c n^{\varepsilon}} N\left(X_{m_{\varepsilon}}\right) ; \hat{T}_{z}>m_{\varepsilon}, v_{n}>m_{\varepsilon}\right) \\
& \leq c_{\varepsilon} \mathrm{e}^{-c_{\varepsilon} n^{\varepsilon}}(1+N(x)) .
\end{aligned}
$$

Putting together (8.12), (8.11) and (8.10) and using (7.3), we obtain (8.9), which completes the proof of the lemma.

Proposition 8.6. 1. For any $\delta \in(0,1), x \in \mathbb{X}$ and $y>0$,

$$
V(x, y) \geq(1-\delta) y-c_{\delta}(1+N(x)) \text {. }
$$

2. For any $x \in \mathbb{X}$,

$$
\lim _{y \rightarrow+\infty} \frac{V(x, y)}{y}=1 .
$$

ProOF. Claim 1. By Lemmas 8.5 and 8.2, we immediately have, with $z=$ $y+r(x)$,

$$
V(x, y) \geq \mathbb{E}_{x}\left(\hat{W}_{k_{0}}(x, z) ; \tau_{y}>k_{0}\right)-\frac{c_{\varepsilon}}{k_{0}^{\varepsilon}}(\max (z, 0)+1+N(x)) .
$$

Using point 1 of Lemma 8.1 and point 2 of Lemma 5.2,

$$
\begin{aligned}
V(x, y) & \geq \mathbb{E}_{x}\left(z+M_{k_{0}} ; \tau_{y}>k_{0}\right)-\frac{c_{\varepsilon}}{k_{0}^{\varepsilon}}(\max (z, 0)+1+N(x)) \\
& \geq z \mathbb{P}_{x}\left(\tau_{y}>k_{0}\right)-c\left(\sqrt{k_{0}}+N(x)\right)-\frac{c_{\varepsilon}}{k_{0}^{\varepsilon}}(\max (z, 0)+1+N(x)) .
\end{aligned}
$$

Since, by the union bound and the Markov inequality,

$$
\mathbb{P}_{x}\left(\tau_{y}>k_{0}\right) \geq \mathbb{P}_{x}\left(\max _{1 \leq k \leq k_{0}}\left|f\left(X_{k}\right)\right|<\frac{y}{k_{0}}\right) \geq 1-\frac{c k_{0}^{2}(1+N(x))}{y},
$$

we obtain that, by the definition of $z$,

$$
V(x, y) \geq\left(1-\frac{c_{\varepsilon}}{k_{0}^{\varepsilon}}\right) y-c_{\varepsilon} k_{0}^{2}(1+N(x)) .
$$

Let $\delta \in(0,1)$. Taking $k_{0}$ large enough, we obtain the desired inequality.

Proof of claim 2. By claim 1, we have $\liminf _{y \rightarrow+\infty} V(x, y) / y \geq 1-\delta$, for any $\delta \in(0,1)$ and $x \in \mathbb{X}$. Taking the limit as $\delta \rightarrow 0$, we obtain the lower bound. Now by (7.4) and (7.3), for any integer $k_{0} \geq 2, y \in \mathbb{R}$ and $z=y+r(x)$,

$$
V(x, y) \leq \hat{W}(x, z) \leq\left(1+\frac{c_{\varepsilon}}{k_{0}^{\varepsilon}}\right)(\max (z, 0)+c N(x))+c_{\varepsilon} k_{0}^{1 / 2} .
$$


Using the definition of $z$, we conclude that

$$
\limsup _{y \rightarrow+\infty} \frac{V(x, y)}{y} \leq \lim _{k_{0} \rightarrow+\infty}\left(1+\frac{c_{\varepsilon}}{k_{0}^{\varepsilon}}\right)=1 .
$$

Now, for any $\gamma>0$, consider the stopping time:

$$
\zeta_{\gamma}:=\inf \left\{k \geq 1:\left|y+S_{k}\right|>\gamma\left(1+N\left(X_{k}\right)\right)\right\} .
$$

The control on the tail of $\zeta_{\gamma}$ is given by the following lemma.

LEMMA 8.7. For any $\gamma>0, x \in \mathbb{X}, y \in \mathbb{R}$ and $n \geq 1$,

$$
\mathbb{P}_{x}\left(\zeta_{\gamma}>n\right) \leq c \mathrm{e}^{-c_{\gamma} n}(1+N(x)) \text {. }
$$

PROOF. The reasoning is very close to that of the proof of the Lemma 6.3. Let $\gamma>0$. Consider the integer $l \geq 1$ which will be chosen later. Define $K:=\left\lfloor\frac{n}{2 l}\right\rfloor$ and introduce the event $A_{k, y}^{\gamma}:=\bigcap_{k^{\prime} \in\{1, \ldots, k\}}\left\{\left|y+S_{k^{\prime} l}\right| \leq \gamma\left(1+N\left(X_{k^{\prime} l}\right)\right)\right\}$. We have

$$
\mathbb{P}_{x}\left(\zeta_{\gamma}>n\right) \leq \mathbb{P}_{x}\left(A_{2 K, y}^{\gamma}\right) \text {. }
$$

By the Markov property,

$$
\begin{aligned}
\mathbb{P}_{x}\left(A_{2 K, y}^{\gamma}\right)= & \int_{\mathbb{X} \times \mathbb{R}} \int_{\mathbb{X} \times \mathbb{R}} \mathbb{P}_{x^{\prime \prime}}\left(A_{1, y^{\prime \prime}}^{\gamma}\right) \mathbb{P}_{x^{\prime}}\left(X_{l} \in \mathrm{d} x^{\prime \prime}, y^{\prime}+S_{l} \in \mathrm{d} y^{\prime \prime}, A_{1, y^{\prime}}^{\gamma}\right) \\
& \times \mathbb{P}_{x}\left(X_{2(K-1) l} \in \mathrm{d} x^{\prime}, y+S_{2(K-1) l} \in \mathrm{d} y^{\prime}, A_{2(K-1), y}^{\gamma}\right) .
\end{aligned}
$$

We write

$$
\begin{aligned}
\mathbb{P}_{x^{\prime \prime}}\left(A_{1, y^{\prime \prime}}^{\gamma}\right) & \leq \mathbb{P}_{x^{\prime \prime}}\left(\left|y^{\prime \prime}+S_{l}\right| \leq 2 \gamma \sqrt{l}\right)+\mathbb{P}_{x^{\prime \prime}}\left(N\left(X_{l}\right)>\sqrt{l}\right) \\
& \leq \mathbb{P}_{x^{\prime \prime}}\left(\frac{-y^{\prime \prime}}{\sqrt{l}}-2 \gamma \leq \frac{S_{l}}{\sqrt{l}} \leq \frac{-y^{\prime \prime}}{\sqrt{l}}+2 \gamma\right)+\mathbb{E}_{x^{\prime \prime}}\left(\frac{N\left(X_{l}\right)}{\sqrt{l}}\right) .
\end{aligned}
$$

By Corollary 4.4 and point 1 of Hypothesis M4, there exists $\varepsilon_{0} \in(0,1 / 4)$ such that, for any $\varepsilon \in\left(0, \varepsilon_{0}\right)$,

$$
\mathbb{P}_{x^{\prime \prime}}\left(A_{1, y^{\prime \prime}}^{\gamma}\right) \leq \int_{\frac{-y^{\prime \prime}}{\sqrt{l}}-2 \gamma}^{\frac{-y^{\prime \prime}}{\sqrt{l}}+2 \gamma} \mathrm{e}^{-\frac{u^{2}}{2 \sigma^{2}}} \frac{\mathrm{d} u}{\sqrt{2 \pi} \sigma}+\frac{2 c_{\varepsilon}}{l^{\varepsilon}}\left(1+N\left(x^{\prime \prime}\right)\right)+\frac{c}{\sqrt{l}}\left(1+N\left(x^{\prime \prime}\right)\right) .
$$

Set $q_{\gamma}:=\int_{-2 \gamma}^{2 \gamma} \mathrm{e}^{-\frac{u^{2}}{2 \sigma^{2}}} \frac{\mathrm{d} u}{\sqrt{2 \pi} \sigma}<1$. From (8.15), we obtain

$$
\begin{aligned}
\mathbb{P}_{x}\left(A_{2 K, y}^{\gamma}\right) \leq & \int_{\mathbb{X} \times \mathbb{R}}\left(q_{\gamma}+\frac{c_{\varepsilon}}{l^{\varepsilon}}+\frac{c_{\varepsilon}}{l^{\varepsilon}} \mathbb{E}_{x^{\prime}}\left(N\left(X_{l}\right)\right)\right) \\
& \times \mathbb{P}_{x}\left(X_{2(K-1) l} \in \mathrm{d} x^{\prime}, y+S_{2(K-1) l} \in \mathrm{d} y^{\prime}, A_{2(K-1), y}^{\gamma}\right) \\
\leq & \left(q_{\gamma}+\frac{c_{\varepsilon}}{l^{\varepsilon}}\right) \mathbb{P}_{x}\left(A_{2(K-1), y}^{\gamma}\right)+c_{\varepsilon} \mathrm{e}^{-c_{\varepsilon} l} \mathbb{E}_{x}\left(N\left(X_{2(K-1) l}\right) ; A_{2(K-1), y}^{\gamma}\right) .
\end{aligned}
$$


For brevity, set $p_{K}=\mathbb{P}_{x}\left(A_{2 K, y}^{\gamma}\right)$ and $E_{K}=\mathbb{E}_{x}\left(N\left(X_{2 K l}\right) ; A_{2 K, y}^{\gamma}\right)$. Then, the previous inequality can be rewritten as

$$
p_{K} \leq\left(q_{\gamma}+\frac{c_{\varepsilon}}{l^{\varepsilon}}\right) p_{K-1}+c_{\varepsilon} \mathrm{e}^{-c_{\varepsilon} l} E_{K-1}
$$

Moreover, from (2.2), we have

$$
E_{K} \leq c p_{K-1}+c \mathrm{e}^{-c 2 l} E_{K-1} .
$$

Using (8.16) and (8.17), we write that

$$
\left(\begin{array}{l}
p_{K} \\
E_{K}
\end{array}\right) \leq A_{l}\left(\begin{array}{c}
p_{K-1} \\
E_{K-1}
\end{array}\right)
$$

where

$$
A_{l}:=\left(\begin{array}{cc}
q_{\gamma}+\frac{c_{\varepsilon}}{l^{\varepsilon}} & c_{\varepsilon} \mathrm{e}^{-c_{\varepsilon} l} \\
c & c \mathrm{e}^{-c l}
\end{array}\right) \underset{l \rightarrow+\infty}{\longrightarrow} A=\left(\begin{array}{cc}
q_{\gamma} & 0 \\
c & 0
\end{array}\right) .
$$

Since the spectral radius $q_{\gamma}$ of $A$ is less than 1 , we can choose $l=l(\varepsilon, \gamma)$ large enough such that the spectral radius $\rho_{\varepsilon, \gamma}$ of $A_{l}$ is less than 1. Iterating (8.18), we get

$$
p_{K} \leq c \rho_{\varepsilon, \gamma}^{K} \max \left(p_{1}, E_{1}\right) \leq c \rho_{\varepsilon, \gamma}^{K}(1+N(x)) .
$$

Taking into account that $K \geq c_{\varepsilon, \gamma} n$, we obtain

$$
\mathbb{P}_{x}\left(A_{2 K, y}^{\gamma}\right) \leq c \mathrm{e}^{-c_{\gamma} n}(1+N(x)) .
$$

Now we shall establish some properties of the set $\mathscr{D}_{\gamma}$ introduced in Section 2. It is easy to see that, for any $\gamma>0$,

$$
\mathscr{D}_{\gamma}=\left\{(x, y) \in \mathbb{X} \times \mathbb{R}: \exists n_{0} \geq 1, \mathbb{P}_{x}\left(\zeta_{\gamma} \leq n_{0}, \tau_{y}>n_{0}\right)>0\right\},
$$

where $\zeta_{\gamma}$ is defined by $(8.14)$.

PROPOSITION 8.8. 1. For any $\gamma_{1} \leq \gamma_{2}$, it holds $\mathscr{D}_{\gamma_{1}} \supseteq \mathscr{D}_{\gamma_{2}}$.

2. For any $\gamma>0$, there exists $c_{\gamma}>0$ such that

$$
\mathscr{D}_{\gamma}^{c} \subseteq\left\{(x, y) \in \mathbb{X} \times \mathbb{R}: \mathbb{P}_{x}\left(\tau_{y}>n\right) \leq \mathrm{e}^{-c_{\gamma} n}(1+N(x)), n \geq 1\right\} .
$$

3. For any $\gamma>0$, the domain of positivity of the function $V$ is included in $\mathscr{D}_{\gamma}$ :

$$
\operatorname{supp}(V)=\{(x, y) \in \mathbb{X} \times \mathbb{R}: V(x, y)>0\} \subseteq \mathscr{D}_{\gamma} .
$$

4. There exists $\gamma_{0}>0$ such that for any $\gamma \geq \gamma_{0}$,

$$
\operatorname{supp}(V)=\mathscr{D}_{\gamma} \text {. }
$$

Moreover,

$$
\left\{(x, y) \in \mathbb{X} \times \mathbb{R}_{+}^{*}: y>\frac{\gamma_{0}}{2}(1+N(x))\right\} \subseteq \operatorname{supp}(V) .
$$


PROOF. Claim 1. For any $\gamma_{1} \leq \gamma_{2}$, we have $\zeta_{\gamma_{1}} \leq \zeta_{\gamma_{2}}$ and claim 1 follows.

Claim 2. Fix $\gamma>0$. By the definition of $\mathscr{D}_{\gamma}$, for any $(x, y) \in \mathscr{D}_{\gamma}^{c}$ and $n \geq 1$,

$$
0=\mathbb{P}_{x}\left(\zeta_{\gamma} \leq n, \tau_{y}>n\right)=\mathbb{P}_{x}\left(\tau_{y}>n\right)-\mathbb{P}_{x}\left(\zeta_{\gamma}>n, \tau_{y}>n\right) .
$$

From this, using Lemma 8.7, we obtain

$$
\mathbb{P}_{x}\left(\tau_{y}>n\right)=\mathbb{P}_{x}\left(\zeta_{\gamma}>n, \tau_{y}>n\right) \leq \mathbb{P}_{x}\left(\zeta_{\gamma}>n\right) \leq \mathrm{e}^{-c_{\gamma} n}(1+N(x)) .
$$

Claim 3. Fix $\gamma>0$. Using the claim 2 and Lemma 5.2, we have, for any $(x, y) \in$ $\mathscr{D}_{\gamma}^{c}, z=y+r(x)$ and $n \geq 1$,

$$
\begin{aligned}
\mathbb{E}_{x}\left(z+M_{n} ; \tau_{y}>n\right) & \leq|z| \mathbb{P}_{x}\left(\tau_{y}>n\right)+\mathbb{E}_{x}^{1 / 2}\left(\left|M_{n}\right|^{2}\right) \mathbb{P}_{x}^{1 / 2}\left(\tau_{y}>n\right) \\
& \leq|z|(1+N(x)) \mathrm{e}^{-c_{\gamma} n}+c \sqrt{n}(1+N(x))^{3 / 2} \mathrm{e}^{-c_{\gamma} n} .
\end{aligned}
$$

Taking the limit when $n \rightarrow+\infty$, by point 1 of Proposition 7.2, we get

$$
V(x, y)=0,
$$

and we conclude that $\mathscr{D}_{\gamma}^{c} \subseteq \operatorname{supp}(V)^{c}$.

Claim 4. By point 1 of Proposition 8.6, taking $\delta=1 / 2$, there exists $\gamma_{0}>0$ such that, for any $x \in \mathbb{X}$ and $y>0$,

$$
V(x, y) \geq \frac{y}{2}-\frac{\gamma_{0}}{4}(1+N(x)) .
$$

Now, fix $(x, y) \in \mathscr{D}_{\gamma_{0}}$ and let $n_{0} \geq 1$ be an integer such that $\mathbb{P}_{x}\left(\zeta_{\gamma_{0}} \leq n_{0}, \tau_{y}>\right.$ $\left.n_{0}\right)>0$. By point 4 of Proposition 7.2,

$$
\begin{aligned}
V(x, y) & =\mathbb{E}_{x}\left(V\left(X_{n_{0}}, y+S_{n_{0}}\right) ; \tau_{y}>n_{0}\right) \\
& \geq \mathbb{E}_{x}\left(V\left(X_{n_{0}}, y+S_{n_{0}}\right) ; \tau_{y}>n_{0}, \zeta_{\gamma_{0}} \leq n_{0}\right) .
\end{aligned}
$$

By the Doob optional stopping theorem, (8.19) and the definition of $\zeta_{\gamma_{0}}$ [see (8.14)],

$$
\begin{aligned}
V(x, y) & \geq \mathbb{E}_{x}\left(V\left(X_{\zeta_{\gamma_{0}}}, y+S_{\zeta_{\gamma_{0}}}\right) ; \tau_{y}>\zeta_{\gamma_{0}}, \zeta_{\gamma_{0}} \leq n_{0}\right) \\
& \geq \frac{1}{2} \mathbb{E}_{x}\left(y+S_{\zeta_{\gamma_{0}}}-\frac{\gamma_{0}}{2}\left(1+N\left(X_{\zeta_{\gamma_{0}}}\right)\right) ; \tau_{y}>\zeta_{\gamma_{0}}, \zeta_{\gamma_{0}} \leq n_{0}\right) \\
& \geq \frac{1}{2} \mathbb{E}_{x}\left(\frac{\gamma_{0}}{2}\left(1+N\left(X_{\zeta_{\gamma_{0}}}\right)\right) ; \tau_{y}>\zeta_{\gamma_{0}}, \zeta_{\gamma_{0}} \leq n_{0}\right) \\
& \geq \frac{\gamma_{0}}{4} \mathbb{P}_{x}\left(\tau_{y}>n_{0}, \zeta_{\gamma_{0}} \leq n_{0}\right) .
\end{aligned}
$$

Now, since $n_{0}$ has been chosen such that the last probability is strictly positive, we get that $V(x, y)>0$. This proves that $\mathscr{D}_{\gamma_{0}} \subseteq \operatorname{supp}(V)$. Using the claims 1 and 3, for any $\gamma \geq \gamma_{0}$, we obtain that $\mathscr{D}_{\gamma} \subseteq \mathscr{D}_{\gamma_{0}} \subseteq \operatorname{supp}(V) \subseteq \mathscr{D}_{\gamma}$ and so $\mathscr{D}_{\gamma}=\mathscr{D}_{\gamma_{0}}=$ $\operatorname{supp}(V)$. Using (8.19) proves the second assertion of claim 4 . 
Proof of Theorem 2.2. Claim 1 is proved by point 1 of Proposition 7.2; claim 2 is proved by point 4 of Proposition 7.2; claim 3 is proved by points 2 and 3 of Proposition 7.2 and by Proposition 8.6; claim 4 is proved by point 4 of Proposition 8.8.

\section{Asymptotic behaviour of the exit time.}

\subsection{Preliminary results.}

LEMMA 9.1. There exists $\varepsilon_{0}>0$ such that, for any $\varepsilon \in\left(0, \varepsilon_{0}\right), x \in \mathbb{X}, y \in \mathbb{R}$ and $z=y+r(x)$,

$$
\begin{aligned}
E_{1} & :=\mathbb{E}_{x}\left(z+M_{v_{n}} ; \tau_{y}>v_{n}, v_{n} \leq\left\lfloor n^{1-\varepsilon}\right\rfloor\right) \\
& \leq c_{\varepsilon}(1+\max (y, 0)+N(x)), \quad n \geq 1, \\
E_{2} & :=\mathbb{E}_{x}\left(z+M_{v_{n}^{\varepsilon^{2}}} ; \tau_{y}>v_{n}^{\varepsilon^{2}}, v_{n}^{\varepsilon^{2}} \leq\left\lfloor n^{1-\varepsilon}\right\rfloor\right) \underset{n \rightarrow \infty}{\longrightarrow} V(x, y) .
\end{aligned}
$$

Moreover, for any $n \geq 1, \varepsilon \in\left(0, \varepsilon_{0}\right), x \in \mathbb{X}$ and $y \in \mathbb{R}$,

$$
\left|E_{2}-V(x, y)\right| \leq \frac{c_{\varepsilon}}{n^{\varepsilon / 8}}(1+\max (y, 0)+N(x)) .
$$

PROOF. Using the fact $\left\{\tau_{y}>v_{n}\right\} \subseteq\left\{\hat{T}_{z}>v_{n}\right\}$ and Lemma 5.4, for $n \geq 1$,

$$
E_{1} \leq \mathbb{E}_{x}\left(z+M_{\left\lfloor n^{1-\varepsilon}\right\rfloor} ; \hat{T}_{z}>\left\lfloor n^{1-\varepsilon}\right\rfloor\right)-J_{21}^{\prime \prime},
$$

where $J_{21}^{\prime \prime}$ is defined in (6.19) and by (6.20) the quantity $-J_{21}^{\prime \prime}$ does not exceed $c_{\varepsilon} \mathrm{e}^{-c_{\varepsilon} n^{\varepsilon}}(1+N(x))$. Again, by Lemma 5.4 and point 1 of Proposition 7.2, we have that $\left(\mathbb{E}_{x}\left(z+M_{n} ; \hat{T}_{z}>n\right)\right)_{n \geq 0}$ is a nondecreasing sequence which converges to $\hat{W}(x, z)$. So, using point 3 of Proposition 7.2 and the fact that $z=y+r(x)$,

$$
E_{1} \leq \hat{W}(x, z)+c_{\varepsilon} \mathrm{e}^{-c_{\varepsilon} n^{\varepsilon}}(1+N(x)) \leq c_{\varepsilon}(1+\max (y, 0)+N(x)) .
$$

By point 4 of Proposition 7.2, we have

$$
\begin{aligned}
V(x, y)= & \mathbb{E}_{x}\left(V\left(X_{n}, y+S_{n}\right) ; \tau_{y}>n, v_{n}^{\varepsilon^{2}} \leq\left\lfloor n^{1-\varepsilon}\right\rfloor\right) \\
& +\mathbb{E}_{x}\left(V\left(X_{n}, y+S_{n}\right) ; \tau_{y}>n, v_{n}^{\varepsilon^{2}}>\left\lfloor n^{1-\varepsilon}\right\rfloor\right) .
\end{aligned}
$$

Using point 3 of Proposition 7.2, for any $k_{0} \geq 2$,

$$
\begin{aligned}
V(x, y) \leq & \mathbb{E}_{x}\left(V\left(X_{v_{n}^{\varepsilon^{2}}}, y+S_{v_{n}^{\varepsilon^{2}}}\right) ; \tau_{y}>v_{n}^{\varepsilon^{2}}, v_{n}^{\varepsilon^{2}} \leq\left\lfloor n^{1-\varepsilon}\right\rfloor\right) \\
& +c \mathbb{E}_{x}\left(\max \left(z+M_{n}, 0\right)+1+N\left(X_{n}\right) ; \tau_{y}>n, v_{n}^{\varepsilon^{2}}>\left\lfloor n^{1-\varepsilon}\right\rfloor\right) \\
\leq & \left(1+\frac{c_{\varepsilon}}{k_{0}^{\varepsilon}}\right) E_{2}+c_{\varepsilon} \mathbb{E}_{x}\left(\sqrt{k_{0}}+N\left(X_{v_{n}^{\varepsilon^{2}}}\right) ; \tau_{y}>v_{n}^{\varepsilon^{2}}, v_{n}^{\varepsilon^{2}} \leq\left\lfloor n^{1-\varepsilon}\right\rfloor\right)
\end{aligned}
$$




$$
\begin{aligned}
& -\underbrace{c_{\varepsilon} \mathbb{E}_{x}\left(z+M_{v_{n}^{\varepsilon^{2}}} ; z+M_{v_{n}^{\varepsilon^{2}}}<0, \tau_{y}>v_{n}^{\varepsilon^{2}}, v_{n}^{\varepsilon^{2}} \leq\left\lfloor n^{1-\varepsilon}\right\rfloor\right)}_{=J_{22}^{\prime}\left(\varepsilon^{2}\right)} \\
& +c \mathbb{E}_{x}\left(z+M_{n}+\left|r\left(X_{n}\right)\right|+1+N\left(X_{n}\right) ; \tau_{y}>n, v_{n}^{\varepsilon^{2}}>\left\lfloor n^{1-\varepsilon}\right\rfloor\right) .
\end{aligned}
$$

From the previous bound, using the Markov property, the bound (2.2) and the approximation (5.1), we get

$$
\begin{aligned}
V(x, y) \leq & \left(1+\frac{c_{\varepsilon}}{k_{0}^{\varepsilon}}\right) E_{2}+J_{22}^{\prime}\left(\varepsilon^{2}\right)+c \underbrace{\mathbb{E}_{x}\left(z+M_{n} ; \hat{T}_{z}>n, v_{n}^{\varepsilon^{2}}>\left\lfloor n^{1-\varepsilon}\right\rfloor\right)}_{=J_{1}\left(\varepsilon^{2}\right)} \\
& +c_{\varepsilon} \mathbb{E}_{x}\left(\sqrt{k_{0}}+\mathrm{e}^{-c n^{\varepsilon^{2}}} N\left(X_{v_{n}}\right) ; \tau_{y}>v_{n}, v_{n} \leq\left\lfloor n^{1-\varepsilon}\right\rfloor\right) \\
& +c \mathbb{E}_{x}\left(1+\mathrm{e}^{-c_{\varepsilon} n} N\left(X_{\left\lfloor n^{1-\varepsilon}\right\rfloor}\right) ; \tau_{y}>\left\lfloor n^{1-\varepsilon}\right\rfloor, v_{n}^{\varepsilon^{2}}>\left\lfloor n^{1-\varepsilon}\right\rfloor\right) .
\end{aligned}
$$

Proceeding in the same way as for the bound (6.25),

$$
\begin{aligned}
J_{22}^{\prime}\left(\varepsilon^{2}\right) & \leq c_{\varepsilon} \mathbb{E}_{x}\left(1+\mathrm{e}^{-c n^{\varepsilon^{2}}} N\left(X_{v_{n}}\right) ; \tau_{y}>v_{n}, v_{n} \leq\left\lfloor n^{1-\varepsilon}\right\rfloor\right) \\
& \leq \frac{c_{\varepsilon}}{n^{1 / 2-\varepsilon}} E_{1}+c_{\varepsilon} \mathrm{e}^{-c_{\varepsilon} n^{\varepsilon^{2}}}(1+N(x)) .
\end{aligned}
$$

Moreover, similarly as for the bound (6.15), we have

$$
J_{1}\left(\varepsilon^{2}\right) \leq c_{\varepsilon} \mathrm{e}^{-c_{\varepsilon} n^{\varepsilon^{2}}}(1+N(x)) .
$$

Taking into account these bounds and using Lemma 6.3,

$$
V(x, y) \leq\left(1+\frac{c_{\varepsilon}}{k_{0}^{\varepsilon}}\right) E_{2}+\frac{c_{\varepsilon} \sqrt{k_{0}}}{n^{1 / 2-\varepsilon}} E_{1}+c_{\varepsilon} \mathrm{e}^{-c_{\varepsilon} n^{\varepsilon^{2}}}(1+N(x)) .
$$

Analogously, by (8.13) and (5.1), we have the lower bound

$$
\begin{aligned}
V(x, y) & \geq \mathbb{E}_{x}\left(V\left(X_{v_{n}^{\varepsilon^{2}}}, y+S_{v_{n}^{\varepsilon^{2}}}\right) ; \tau_{y}>v_{n}^{\varepsilon^{2}}, v_{n}^{\varepsilon^{2}} \leq\left\lfloor n^{1-\varepsilon}\right\rfloor\right) \\
& \geq\left(1-\frac{c_{\varepsilon}}{k_{0}^{\varepsilon}}\right) E_{2}-c_{\varepsilon} k_{0}^{2} \mathbb{E}_{x}\left(1+N\left(X_{v_{n}^{\varepsilon^{2}}}\right) ; \tau_{y}>v_{n}^{\varepsilon^{2}}, v_{n}^{\varepsilon^{2}} \leq\left\lfloor n^{1-\varepsilon}\right\rfloor\right) \\
& \geq\left(1-\frac{c_{\varepsilon}}{k_{0}^{\varepsilon}}\right) E_{2}-\frac{c_{\varepsilon} k_{0}^{2}}{n^{1 / 2-\varepsilon}} E_{1}-c_{\varepsilon} k_{0}^{2} \mathrm{e}^{-c_{\varepsilon} n^{\varepsilon^{2}}}(1+N(x)) .
\end{aligned}
$$

Taking $k_{0}=n^{1 / 4-\varepsilon}$ in (9.3) and (9.2), we conclude that, for any $\varepsilon \in(0,1 / 8)$,

$$
\left|V(x, y)-E_{2}\right| \leq \frac{c_{\varepsilon}}{n^{\varepsilon / 8}} E_{2}+\frac{c_{\varepsilon}}{n^{\varepsilon}}\left(E_{1}+1+N(x)\right) .
$$

Again, using (9.3),

$$
\left|V(x, y)-E_{2}\right| \leq \frac{c_{\varepsilon}}{n^{\varepsilon / 8}} V(x, y)+\frac{c_{\varepsilon}}{n^{\varepsilon}}\left(E_{1}+1+N(x)\right) .
$$


Finally, employing (9.1) and (7.5),

$$
\left|V(x, y)-E_{2}\right| \leq \frac{c_{\varepsilon}}{n^{\varepsilon / 8}}(1+\max (y, 0)+N(x)) .
$$

LEMMA 9.2. There exists $\varepsilon_{0}>0$ such that, for any $\varepsilon \in\left(0, \varepsilon_{0}\right), x \in \mathbb{X}, y \in \mathbb{R}$ and $n \geq 1$,

$$
\mathbb{P}_{x}\left(\tau_{y}>n\right) \leq \frac{c_{\varepsilon}}{n^{1 / 2-\varepsilon}}(1+\max (y, 0)+N(x)) .
$$

Moreover, summing this bound, for any $\varepsilon \in\left(0, \varepsilon_{0}\right), x \in \mathbb{X}, y \in \mathbb{R}$ and $n \geq 1$, we have

$$
\sum_{k=1}^{\left\lfloor n^{1-\varepsilon}\right\rfloor} \mathbb{P}_{x}\left(\tau_{y}>k\right) \leq c_{\varepsilon}(1+\max (y, 0)+N(x)) n^{1 / 2+\varepsilon / 2}
$$

PROOF. Using Lemma 6.3 and Lemma 9.1, with $z=y+r(x)$ and $n \geq 1$,

$$
\begin{aligned}
\mathbb{P}_{x}\left(\tau_{y}>n\right) & \leq \mathbb{P}_{x}\left(\tau_{y}>n, v_{n} \leq\left\lfloor n^{1-\varepsilon}\right\rfloor\right)+\mathbb{P}_{x}\left(\hat{T}_{z}>n, v_{n}>\left\lfloor n^{1-\varepsilon}\right\rfloor\right) \\
& \leq \mathbb{E}_{x}\left(\frac{z+M_{v_{n}}}{n^{1 / 2-\varepsilon}} ; \tau_{y}>n, v_{n} \leq\left\lfloor n^{1-\varepsilon}\right\rfloor\right)+c_{\varepsilon} \mathrm{e}^{-c_{\varepsilon} n^{\varepsilon}}(1+N(x)) \\
& \leq \frac{c_{\varepsilon}}{n^{1 / 2-\varepsilon}}(1+\max (y, 0)+N(x)) .
\end{aligned}
$$

LEMmA 9.3. There exists $\varepsilon_{0}>0$ such that, for any $\varepsilon \in\left(0, \varepsilon_{0}\right), x \in \mathbb{X}, y \in \mathbb{R}$ and $z=y+r(x)$,

$$
E_{3}:=\mathbb{E}_{x}\left(z+M_{v_{n}} ; z+M_{v_{n}}>n^{1 / 2-\varepsilon / 2}, \tau_{y}>v_{n}, v_{n} \leq\left\lfloor n^{1-\varepsilon}\right\rfloor\right) \underset{n \rightarrow+\infty}{\longrightarrow} 0 .
$$

More precisely, for any $n \geq 1, \varepsilon \in\left(0, \varepsilon_{0}\right), x \in \mathbb{X}, y \in \mathbb{R}$ and $z=y+r(x)$,

$$
E_{3} \leq c_{\varepsilon} \frac{\max (y, 0)+\left(1+y \mathbb{1}_{\left\{y>n^{1 / 2-2 \varepsilon}\right\}}+N(x)\right)^{2}}{n^{\varepsilon}} .
$$

PROOF. Notice that when $v_{n} \neq 1$ the following inclusion holds:

$$
\left\{z+M_{v_{n}}>n^{1 / 2-\varepsilon / 2}\right\} \subseteq\left\{\xi_{v_{n}}>n^{1 / 2-\varepsilon / 2}-n^{1 / 2-\varepsilon} \geq c_{\varepsilon} n^{1 / 2-\varepsilon / 2}\right\} .
$$

Therefore,

$$
\begin{aligned}
E_{3} \leq \underbrace{\mathbb{E}_{x}\left(z+M_{v_{n}} ; v_{n} \leq 2\left\lfloor n^{\varepsilon}\right\rfloor\right)}_{=: E_{30}} \\
+\underbrace{\sum_{k=2\left\lfloor n^{\varepsilon}\right\rfloor+1}^{\left\lfloor n^{1-\varepsilon}\right\rfloor} \mathbb{E}_{x}\left(z+M_{k} ; \xi_{k}>c_{\varepsilon} n^{1 / 2-\varepsilon / 2}, \tau_{y}>k, v_{n}=k\right)}_{=: E_{31}} .
\end{aligned}
$$


Bound of $E_{30}$. For $y \leq n^{1 / 2-2 \varepsilon}$, by (6.5), the Markov inequality and Lemma 5.2,

$$
\mathbb{P}_{x}\left(v_{n} \leq 2\left\lfloor n^{\varepsilon}\right\rfloor\right) \leq \sum_{k=1}^{2\left\lfloor n^{\varepsilon}\right\rfloor} \mathbb{P}_{x}\left(r(x)+M_{k}>n^{1 / 2-\varepsilon}-y\right) \leq \frac{c_{\varepsilon}(1+N(x))}{n^{1 / 2-3 \varepsilon}}
$$

For $y>n^{1 / 2-2 \varepsilon}$, in the same way, we have $\mathbb{P}_{x}\left(v_{n} \leq 2\left\lfloor n^{\varepsilon}\right\rfloor\right) \leq \frac{c_{\varepsilon}(1+y+N(x))}{n^{1 / 2-3 \varepsilon}}$. Putting together these bounds, we get, for any $y \in \mathbb{R}$,

$$
\mathbb{P}_{x}\left(v_{n} \leq 2\left\lfloor n^{\varepsilon}\right\rfloor\right) \leq \frac{c_{\varepsilon}\left(1+y \mathbb{1}_{\left\{y>n^{1 / 2-2 \varepsilon}\right\}}+N(x)\right)}{n^{1 / 2-3 \varepsilon}} .
$$

Using Lemma 5.2,

$$
\begin{aligned}
E_{30} & \leq z \mathbb{P}_{x}\left(v_{n} \leq 2\left\lfloor n^{\varepsilon}\right\rfloor\right)+\sum_{k=1}^{2\left\lfloor n^{\varepsilon}\right\rfloor} \mathbb{E}_{x}^{1 / 2}\left(\left|M_{k}\right|^{2}\right) \mathbb{P}_{x}^{1 / 2}\left(v_{n} \leq 2\left\lfloor n^{\varepsilon}\right\rfloor\right) \\
& \leq \frac{c_{\varepsilon}\left(1+y \mathbb{1}_{\left\{y>n^{1 / 2-2 \varepsilon}\right\}}+N(x)\right)^{2}}{n^{\varepsilon}} .
\end{aligned}
$$

Bound of $E_{31}$. Changing the index of summation $\left(j=k-\left\lfloor n^{\varepsilon}\right\rfloor\right)$ and using the Markov property,

$$
\begin{aligned}
& E_{31} \leq \underbrace{\sum_{j=\left\lfloor n^{\varepsilon}\right\rfloor+1}^{\left\lfloor n^{1-\varepsilon}\right\rfloor} \int_{\mathbb{X} \times \mathbb{R}} \max \left(z^{\prime}, 0\right) \mathbb{P}_{x^{\prime}}\left(\xi_{\left\lfloor n^{\varepsilon}\right\rfloor}>c_{\varepsilon} n^{1 / 2-\varepsilon / 2}\right)}_{=: E_{32}} \times \mathbb{P}_{x}\left(X_{j} \in \mathrm{d} x^{\prime}, z+M_{j} \in \mathrm{d} z^{\prime}, \tau_{y}>j\right) \\
&+\underbrace{\sum_{j=\left\lfloor n^{\varepsilon}\right\rfloor+1}^{\left\lfloor n^{1-\varepsilon}\right\rfloor} \int_{\mathbb{X} \times \mathbb{R}} \mathbb{E}_{x^{\prime}}^{1 / 2}\left(\left|M_{\left\lfloor n^{\varepsilon}\right\rfloor}\right|^{2}\right) \mathbb{P}_{x^{\prime}}^{1 / 2}\left(\xi_{\left\lfloor n^{\varepsilon}\right\rfloor}>c_{\varepsilon} n^{1 / 2-\varepsilon / 2}\right)}_{x=: E_{33}}
\end{aligned}
$$

Bound of $E_{32}$. Using (5.2), the Markov inequality and (2.3) with $l=$ $\left\lfloor c_{\varepsilon} n^{1 / 2-\varepsilon / 2}\right\rfloor$,

$$
\begin{aligned}
\mathbb{P}_{x^{\prime}}\left(\xi_{\left\lfloor n^{\varepsilon}\right\rfloor}>c_{\varepsilon} n^{1 / 2-\varepsilon / 2}\right) \leq & \mathbb{P}_{x^{\prime}}\left(N\left(X_{\left\lfloor n^{\varepsilon}\right\rfloor}\right)>c_{\varepsilon} n^{1 / 2-\varepsilon / 2}\right) \\
& +\mathbb{P}_{x^{\prime}}\left(N\left(X_{\left\lfloor n^{\varepsilon}\right\rfloor-1}\right)>c_{\varepsilon} n^{1 / 2-\varepsilon / 2}\right) \\
\leq & \frac{1}{l} \mathbb{E}_{x^{\prime}}\left(N_{l}\left(X_{\left\lfloor n^{\varepsilon}\right\rfloor}\right)\right)+\frac{1}{l} \mathbb{E}_{x^{\prime}}\left(N_{l}\left(X_{\left\lfloor n^{\varepsilon}\right\rfloor-1}\right)\right) \\
\leq & \frac{c}{l^{2+\beta}}+\frac{c}{l} \mathrm{e}^{-c n^{\varepsilon}}\left(1+N\left(x^{\prime}\right)\right) .
\end{aligned}
$$


Choosing $\varepsilon>0$ small enough we find that

$$
\mathbb{P}_{x^{\prime}}\left(\xi_{\left\lfloor n^{\varepsilon}\right\rfloor}>c_{\varepsilon} n^{1 / 2-\varepsilon / 2}\right) \leq \frac{c_{\varepsilon}}{n^{1+\beta / 4}}+c_{\varepsilon} \mathrm{e}^{-c_{\varepsilon} n^{\varepsilon}} N\left(x^{\prime}\right) .
$$

By the definition of $E_{32}$ in (9.7),

$$
\begin{aligned}
E_{32} \leq & \frac{c_{\varepsilon}}{n^{1+\beta / 4}} \sum_{j=\left\lfloor n^{\varepsilon}\right\rfloor+1}^{\left\lfloor n^{1-\varepsilon}\right\rfloor}\left[\mathbb{E}_{x}\left(z+M_{j} ; \tau_{y}>j\right)+\mathbb{E}_{x}\left(\left|r\left(X_{j}\right)\right|\right)\right] \\
& +c_{\varepsilon} \mathrm{e}^{-c_{\varepsilon} n^{\varepsilon}} \sum_{j=\left\lfloor n^{\varepsilon}\right\rfloor+1}^{\left\lfloor n^{1-\varepsilon}\right\rfloor}\left[\max (z, 0) \mathbb{E}_{x}\left(N\left(X_{j}\right)\right)+\mathbb{E}_{x}^{1 / 2}\left(\left|M_{j}\right|^{2}\right) \mathbb{E}_{x}^{1 / 2}\left(N\left(X_{j}\right)^{2}\right)\right] .
\end{aligned}
$$

Using (6.29), Lemma 5.2 and point 1 of Hypothesis M4, we find that

$$
E_{32} \leq c_{\varepsilon} \frac{\max (y, 0)+\left(1+y \mathbb{1}_{\left\{y>n^{1 / 2-2 \varepsilon}\right\}}+N(x)\right)(1+N(x))}{n^{\beta / 4}} .
$$

Bound of $E_{33}$. Using (9.8) and Lemma 5.2, we have

$$
E_{33} \leq \sum_{j=\left\lfloor n^{\varepsilon}\right\rfloor+1}^{\left\lfloor n^{1-\varepsilon}\right\rfloor} \mathbb{E}_{x}\left(n^{\varepsilon / 2}\left(1+N\left(X_{j}\right)\right)\left(\frac{c_{\varepsilon}}{n^{1 / 2+\beta / 8}}+c_{\varepsilon} \mathrm{e}^{-c_{\varepsilon} n^{\varepsilon}} N\left(X_{j}\right)^{1 / 2}\right) ; \tau_{y}>j\right)
$$

By the Markov property,

$$
\begin{aligned}
E_{33} \leq & c_{\varepsilon} \mathrm{e}^{-c_{\varepsilon} n^{\varepsilon}}(1+N(x))^{3 / 2} \\
& +\frac{c_{\varepsilon}}{n^{1 / 2+\beta / 8-\varepsilon / 2}} \sum_{j=1}^{\left\lfloor n^{1-\varepsilon}\right\rfloor} \mathbb{E}_{x}\left(1+\mathrm{e}^{-c n^{\varepsilon}} N\left(X_{j}\right) ; \tau_{y}>j\right) .
\end{aligned}
$$

Using Lemma 9.2,

$$
E_{33} \leq c_{\varepsilon} \frac{\max (y, 0)+(1+N(x))^{3 / 2}}{n^{\beta / 8-3 \varepsilon / 2}} .
$$

With (9.10), (9.9) and (9.7), for $\varepsilon>0$ small enough, we find that

$$
E_{31} \leq c_{\varepsilon} \frac{\max (y, 0)+\left(1+y \mathbb{1}_{\left\{y>n^{1 / 2-2 \varepsilon}\right\}}+N(x)\right)(1+N(x))}{n^{\varepsilon}} .
$$

This bound, together with (9.6) and (9.4), proves the lemma.

LEMmA 9.4. There exists $\varepsilon_{0}>0$ such that, for any $\varepsilon \in\left(0, \varepsilon_{0}\right), x \in \mathbb{X}, y \in \mathbb{R}$ and $z=y+r(x)$,

$$
E_{4}:=\mathbb{E}_{x}\left(z+M_{v_{n}^{\varepsilon^{2}}} ; z+M_{\nu_{n}^{\varepsilon^{2}}}>n^{1 / 2-\varepsilon / 4}, \tau_{y}>v_{n}^{\varepsilon^{2}}, v_{n}^{\varepsilon^{2}} \leq\left\lfloor n^{1-\varepsilon}\right\rfloor\right) \underset{n \rightarrow+\infty}{\longrightarrow} 0 .
$$


More precisely, for any $n \geq 1, \varepsilon \in\left(0, \varepsilon_{0}\right), x \in \mathbb{X}, y \in \mathbb{R}$ and $z=y+r(x)$,

$$
E_{4} \leq c_{\varepsilon} \frac{\max (y, 0)+\left(1+y \mathbb{1}_{\left\{y>n^{1 / 2-2 \varepsilon}\right\}}+N(x)\right)^{2}}{n^{\varepsilon / 2}} .
$$

PROOF. We shall apply Lemma 9.3. For this we write, for any $n \geq 1$,

$$
\begin{aligned}
E_{4}= & \mathbb{E}_{x}\left(z+M_{v_{n}^{\varepsilon^{2}}} ; z+M_{v_{n}^{\varepsilon^{2}}}>n^{1 / 2-\varepsilon / 4}, z+M_{v_{n}}>n^{1 / 2-\varepsilon / 2},\right. \\
\underbrace{\left.\tau_{y}>v_{n}^{\varepsilon^{2}}, v_{n}^{\varepsilon^{2}} \leq\left\lfloor n^{1-\varepsilon}\right\rfloor\right)}_{=: E_{41}} & \underbrace{\mathbb{E}_{x}\left(z+M_{v_{n}^{\varepsilon^{2}}} ; z+M_{v_{n}^{\varepsilon^{2}}}>n^{1 / 2-\varepsilon / 4}, z+v_{n}^{\varepsilon^{2}}, v_{n}^{\varepsilon^{2}} \leq\left\lfloor n^{1-\varepsilon}\right\rfloor\right)}_{=: E_{42}} .
\end{aligned}
$$

Bound of $E_{41}$. By the Markov property,

$$
\begin{aligned}
E_{41}= & \sum_{k=1}^{\left\lfloor n^{1-\varepsilon}\right\rfloor-\left\lfloor n^{\varepsilon^{2}}\right\rfloor} \int_{\mathbb{X} \times \mathbb{R}} \mathbb{E}_{x^{\prime}}\left(z^{\prime}+M_{\left\lfloor n^{\varepsilon^{2}}\right\rfloor} ; z^{\prime}+M_{\left\lfloor n^{\left.\varepsilon^{2}\right\rfloor}\right.}>n^{1 / 2-\varepsilon / 4}, \tau_{y^{\prime}}>\left\lfloor n^{\left.\left.\varepsilon^{2}\right\rfloor\right)}\right.\right. \\
& \times \mathbb{P}_{x}\left(X_{k} \in \mathrm{d} x^{\prime}, z+M_{k} \in \mathrm{d} z^{\prime}, z+M_{k}>n^{1 / 2-\varepsilon / 2}, \tau_{y}>k, v_{n}=k\right),
\end{aligned}
$$

where $y^{\prime}=z^{\prime}-r\left(x^{\prime}\right)$. Moreover, for any $x^{\prime} \in \mathbb{X}, z^{\prime} \in \mathbb{R}$, using (6.29), we have

$$
\begin{aligned}
& \mathbb{E}_{x^{\prime}}\left(z^{\prime}+M_{\left\lfloor n^{\varepsilon^{2}}\right\rfloor} ; z^{\prime}+M_{\left\lfloor n^{\varepsilon^{2}}\right\rfloor}>n^{1 / 2-\varepsilon / 4}, \tau_{y^{\prime}}>\left\lfloor n^{\varepsilon^{2}}\right\rfloor\right) \\
& \leq \mathbb{E}_{x^{\prime}}\left(z^{\prime}+M_{\left\lfloor n^{\varepsilon^{2}}\right\rfloor} ; z^{\prime}+M_{\left\lfloor n^{\varepsilon^{2}}\right\rfloor}>0, \tau_{y^{\prime}}>\left\lfloor n^{\varepsilon^{2}}\right\rfloor\right) \\
& \leq \mathbb{E}_{x^{\prime}}\left(z^{\prime}+M_{\left\lfloor n^{\varepsilon^{2}}\right\rfloor} ; \tau_{y^{\prime}}>\left\lfloor n^{\varepsilon^{2}}\right\rfloor\right)+\mathbb{E}_{x^{\prime}}\left(\left|r\left(X_{n^{\varepsilon^{2}}}\right)\right|\right) \\
& \leq c_{\varepsilon} \max \left(z^{\prime}, 0\right)+c_{\varepsilon}\left(1+N\left(x^{\prime}\right)\right) \text {. }
\end{aligned}
$$

Consequently,

$$
\begin{aligned}
E_{41} \leq & c_{\varepsilon} E_{3}+c_{\varepsilon} \mathbb{E}_{x}\left(1+N\left(X_{v_{n}}\right) ; z+M_{v_{n}}>n^{1 / 2-\varepsilon / 2}, \tau_{y}>v_{n}, v_{n} \leq\left\lfloor n^{1-\varepsilon}\right\rfloor\right) \\
\leq & 2 c_{\varepsilon} E_{3}+c_{\varepsilon} \mathbb{E}_{x}\left(N\left(X_{v_{n}}\right) ; N\left(X_{v_{n}}\right)>n^{1 / 2-\varepsilon}, \tau_{y}>v_{n}, v_{n} \leq\left\lfloor n^{1-\varepsilon}\right\rfloor\right) \\
(9.12) \quad & +c_{\varepsilon} \mathbb{E}_{x}\left(n^{1 / 2-\varepsilon} ; N\left(X_{v_{n}}\right) \leq n^{1 / 2-\varepsilon}, z+M_{v_{n}}>n^{1 / 2-\varepsilon / 2},\right. \\
& \left.\tau_{y}>v_{n}, v_{n} \leq\left\lfloor n^{1-\varepsilon}\right\rfloor\right) \\
\leq & 3 c_{\varepsilon} E_{3}+c_{\varepsilon} \underbrace{\mathbb{E}_{x}\left(N\left(X_{v_{n}}\right) ; N\left(X_{v_{n}}\right)>n^{1 / 2-\varepsilon}, \tau_{y}>v_{n}, v_{n} \leq\left\lfloor n^{1-\varepsilon}\right\rfloor\right)}_{=: E_{41}^{\prime}} .
\end{aligned}
$$


Denoting $l=\left\lfloor n^{1 / 2-\varepsilon}\right\rfloor$ and using point 1 of M4 and (2.3), we have

$$
\begin{aligned}
E_{41}^{\prime} & \leq \mathbb{E}_{x}\left(\frac{N\left(X_{v_{n}}\right)^{2}}{n^{1 / 2-\varepsilon}} ; v_{n} \leq\left\lfloor n^{\varepsilon}\right\rfloor\right)+\sum_{k=\left\lfloor n^{\varepsilon}\right\rfloor+1}^{\left\lfloor n^{1-\varepsilon}\right\rfloor} \mathbb{E}_{x}\left(N_{l}\left(X_{k}\right) ; \tau_{y}>k, v_{n}=k\right) \\
& \leq \frac{c n^{\varepsilon}(1+N(x))^{2}}{n^{1 / 2-\varepsilon}}+\sum_{k=1}^{\left\lfloor n^{1-\varepsilon}\right\rfloor}\left[\frac{c}{l^{1+\beta}} \mathbb{P}_{x}\left(\tau_{y}>k\right)+c \mathrm{e}^{-c n^{\varepsilon}} \mathbb{E}_{x}\left(1+N\left(X_{k}\right)\right)\right] .
\end{aligned}
$$

Using Lemma 9.2 and taking $\varepsilon>0$ small enough,

$$
E_{41}^{\prime} \leq c_{\varepsilon} \frac{\max (y, 0)+(1+N(x))^{2}}{n^{\min (1, \beta) / 4}} .
$$

In conjunction with Lemma 9.3, from (9.12) we obtain that, for some $\varepsilon>0$,

$$
E_{41} \leq c_{\varepsilon} \frac{\max (y, 0)+\left(1+y \mathbb{1}_{\left\{y>n^{1 / 2-2 \varepsilon}\right\}}+N(x)\right)^{2}}{n^{\varepsilon}} .
$$

Bound of $E_{42}$. For any $z^{\prime} \in\left(0, n^{1 / 2-\varepsilon / 2}\right]$, we have $\left(z^{\prime}+M_{\left\lfloor n^{\varepsilon^{2}}\right\rfloor}\right) \mathbb{P}_{x^{\prime}}\left(z^{\prime}+M_{\left\lfloor n^{\varepsilon^{2}}\right\rfloor}>n^{1 / 2-\varepsilon / 4}\right) \leq z^{\prime} \mathbb{P}_{x^{\prime}}\left(M_{\left\lfloor n^{\left.\varepsilon^{2}\right\rfloor}\right.}>c_{\varepsilon} n^{1 / 2-\varepsilon / 4}\right)+\left|M_{\left\lfloor n^{\varepsilon^{2}}\right\rfloor}\right|$.

Therefore, by the Markov property,

$$
\begin{aligned}
& E_{42} \leq \underbrace{}_{\mathbb{X} \times \mathbb{R}} z^{\prime} \mathbb{P}_{x^{\prime}}\left(M_{\left\lfloor n^{\varepsilon^{2}}\right\rfloor}>c_{\varepsilon} n^{1 / 2-\varepsilon / 4}\right) \mathbb{P}_{x}\left(X_{v_{n}} \in \mathrm{d} x^{\prime}, z+M_{v_{n}} \in \mathrm{d} z^{\prime},\right. \\
&\left.z+M_{v_{n}} \leq n^{1 / 2-\varepsilon / 2}, \tau_{y}>v_{n}, v_{n} \leq\left\lfloor n^{1-\varepsilon}\right\rfloor\right)
\end{aligned}
$$

Bound of $E_{43}$. Using Lemma 5.2,

$$
\mathbb{P}_{x^{\prime}}\left(M_{\left\lfloor n^{\left.\varepsilon^{2}\right\rfloor}\right.}>c_{\varepsilon} n^{1 / 2-\varepsilon / 4}\right) \leq \frac{c_{\varepsilon} n^{\varepsilon^{2}}\left(1+N\left(x^{\prime}\right)\right)}{n^{1 / 2-\varepsilon / 4}} .
$$

Therefore, we have

$$
\begin{aligned}
E_{43} \leq & \mathbb{E}_{x}\left(\frac{c_{\varepsilon}}{n^{3 \varepsilon / 4-\varepsilon^{2}}}\left(z+M_{v_{n}}\right) \mathbb{1}_{\left\{N\left(X_{v_{n}}\right) \leq n^{1 / 2-\varepsilon}\right\}}+\frac{c_{\varepsilon}}{n^{\varepsilon / 4-\varepsilon^{2}}} N\left(X_{v_{n}}\right) \mathbb{1}_{\left\{N\left(X_{v_{n}}\right)>n^{1 / 2-\varepsilon}\right\}}\right. \\
& \left.z+M_{v_{n}} \leq n^{1 / 2-\varepsilon / 2}, \tau_{y}>v_{n}, v_{n} \leq\left\lfloor n^{1-\varepsilon}\right\rfloor\right) \\
\leq & \frac{c_{\varepsilon}}{n^{3 \varepsilon / 4-\varepsilon^{2}}} E_{1}+\frac{c_{\varepsilon}}{n^{\varepsilon / 4-\varepsilon^{2}}} E_{41}^{\prime} .
\end{aligned}
$$


By Lemma 9.1 and (9.13), we obtain for some small $\varepsilon>0$,

$$
E_{43} \leq c_{\varepsilon} \frac{\max (y, 0)+(1+N(x))^{2}}{n^{\varepsilon / 2}} .
$$

Bound of $E_{44}$. Again by Lemma 5.2, $\mathbb{E}_{x^{\prime}}\left(\left|M_{\left\lfloor n^{\left.\varepsilon^{2}\right\rfloor}\right.}\right|\right) \leq n^{\varepsilon^{2}}\left(1+N\left(x^{\prime}\right)\right)$. Consequently,

$$
\begin{aligned}
E_{44} \leq & \frac{c_{\varepsilon}}{n^{\varepsilon-\varepsilon^{2}}} \mathbb{E}_{x}\left(z+M_{v_{n}} ; N\left(X_{v_{n}}\right) \leq n^{1 / 2-2 \varepsilon}, \tau_{y}>v_{n}, v_{n} \leq\left\lfloor n^{1-\varepsilon}\right\rfloor\right) \\
& +c_{\varepsilon} n^{\varepsilon^{2}} \mathbb{E}_{x}\left(N\left(X_{v_{n}}\right) ; N\left(X_{v_{n}}\right)>n^{1 / 2-2 \varepsilon}, \tau_{y}>v_{n}, v_{n} \leq\left\lfloor n^{1-\varepsilon}\right\rfloor\right) .
\end{aligned}
$$

Proceeding exactly as in the proof of the bound of $E_{41}^{\prime}$ but with $l=\left\lfloor n^{1 / 2-2 \varepsilon}\right\rfloor$, we obtain, by Lemma 9.1,

$$
E_{44} \leq c_{\varepsilon} \frac{\max (y, 0)+(1+N(x))^{2}}{n^{\varepsilon / 2}} .
$$

Putting together this bound with (9.16) and (9.15), we find that

$$
E_{42} \leq c_{\varepsilon} \frac{\max (y, 0)+(1+N(x))^{2}}{n^{\varepsilon / 2}} .
$$

So, using (9.11) and (9.14), we obtain the second assertion. The first one is an easy consequence of the second one.

The following results are similar to that provided by Lemmas 9.1 and 9.4 (see $E_{2}$ and $E_{4}$ respectively).

LEMMA 9.5. There exists $\varepsilon_{0}>0$ such that, for any $\varepsilon \in\left(0, \varepsilon_{0}\right), x \in \mathbb{X}$ and $y \in \mathbb{R}$,

$$
\begin{aligned}
& F_{2}:=\mathbb{E}_{x}\left(y+S_{v_{n}^{\varepsilon^{2}}} ; \tau_{y}>v_{n}^{\varepsilon^{2}}, v_{n}^{\varepsilon^{2}} \leq\left\lfloor n^{1-\varepsilon}\right\rfloor\right) \underset{n \rightarrow \infty}{\longrightarrow} V(x, y), \\
& F_{4}:=\mathbb{E}_{x}\left(y+S_{v_{n}^{\varepsilon^{2}}} ; y+S_{v_{n}^{\varepsilon^{2}}}>n^{1 / 2-\varepsilon / 8}, \tau_{y}>v_{n}^{\varepsilon^{2}}, v_{n}^{\varepsilon^{2}} \leq\left\lfloor n^{1-\varepsilon}\right\rfloor\right) \underset{n \rightarrow+\infty}{\longrightarrow} 0 .
\end{aligned}
$$

More precisely, for any $n \geq 1, \varepsilon \in\left(0, \varepsilon_{0}\right), x \in \mathbb{X}$ and $y \in \mathbb{R}$,

$$
\left|F_{2}-V(x, y)\right| \leq \frac{c_{\varepsilon}}{n^{\varepsilon / 8}}(1+\max (y, 0)+N(x))
$$

and

$$
F_{4} \leq c_{\varepsilon} \frac{\max (y, 0)+\left(1+y \mathbb{1}_{\left\{y>n^{1 / 2-2 \varepsilon}\right\}}+N(x)\right)^{2}}{n^{\varepsilon / 2}} .
$$


PROOF. By (5.1), for any $n \geq 1$,

$$
\left|F_{2}-E_{2}\right| \leq \underbrace{\mathbb{E}_{x}\left(\left|r\left(X_{v_{n}^{\varepsilon^{2}}}\right)\right| ; \tau_{y}>v_{n}^{\varepsilon^{2}}, v_{n}^{\varepsilon^{2}} \leq\left\lfloor n^{1-\varepsilon}\right\rfloor\right)}_{=: F_{2}^{\prime}} .
$$

Using the Markov property, the definition of $v_{n}$ and Lemma 9.1,

$$
\begin{aligned}
F_{2}^{\prime} & \leq c \mathbb{E}_{x}\left(1+\mathrm{e}^{-c n^{\varepsilon^{2}}} N\left(X_{v_{n}}\right) ; \tau_{y}>v_{n}, v_{n} \leq\left\lfloor n^{1-\varepsilon}\right\rfloor\right) \\
& \leq \frac{c}{n^{1 / 2-\varepsilon}} E_{1}+c \mathrm{e}^{-c n^{\varepsilon^{2}}}(1+N(x)) \\
& \leq \frac{c_{\varepsilon}}{n^{1 / 2-\varepsilon}}(1+\max (y, 0)+N(x)) .
\end{aligned}
$$

Therefore, by Lemma 9.1,

$$
\left|F_{2}-V(x, y)\right| \leq\left|E_{2}-V(x, y)\right|+F_{2}^{\prime} \leq \frac{c_{\varepsilon}}{n^{\varepsilon / 8}}(1+\max (y, 0)+N(x)) .
$$

Now we shall control $F_{4}$. Recall the notation $z=y+r(x)$. By equation (5.1), we note that on the event

$$
\left\{z+M_{v_{n}^{\varepsilon^{2}}} \leq n^{1 / 2-\varepsilon / 4}\right\} \cap\left\{y+S_{v_{n}^{\varepsilon^{2}}}>n^{1 / 2-\varepsilon / 8}\right\}
$$

we have $\left|r\left(X_{v_{n}^{\varepsilon^{2}}}\right)\right|>c_{\varepsilon} n^{1 / 2-\varepsilon / 8}$. Therefore,

$$
y+S_{v_{n}^{\varepsilon^{2}}} \leq n^{1 / 2-\varepsilon / 4}-r\left(X_{v_{n}^{\varepsilon^{2}}}\right) \leq\left(\frac{c_{\varepsilon}}{n^{\varepsilon / 8}}+1\right)\left|r\left(X_{v_{n}^{\varepsilon^{2}}}\right)\right|
$$

which implies that

$$
F_{4} \leq \mathbb{E}_{x}\left(y+S_{v_{n}^{2}} ; z+M_{v_{n}^{\varepsilon^{2}}}>n^{1 / 2-\varepsilon / 4}, \tau_{y}>v_{n}^{\varepsilon^{2}}, v_{n}^{\varepsilon^{2}} \leq\left\lfloor n^{1-\varepsilon}\right\rfloor\right)+c_{\varepsilon} F_{2}^{\prime} .
$$

By (5.1), Lemma 9.4 and (9.17), we conclude that

$$
F_{4} \leq E_{4}+F_{2}^{\prime}+c_{\varepsilon} F_{2}^{\prime} \leq c_{\varepsilon} \frac{\max (y, 0)+\left(1+y \mathbb{1}_{\left\{y>n^{1 / 2-2 \varepsilon}\right\}}+N(x)\right)^{2}}{n^{\varepsilon / 2}} .
$$

9.2. Proof of Theorem 2.3. Assume that $(x, y) \in \mathbb{X} \times \mathbb{R}$. Let $\left(B_{t}\right)_{t \geq 0}$ be the Brownian motion defined by Proposition 4.3. For any $k \geq 1$, consider the event

$$
A_{k}=\left\{\sup _{0 \leq t \leq 1}\left|S_{\lfloor t k\rfloor}-\sigma B_{t k}\right| \leq k^{1 / 2-2 \varepsilon}\right\}
$$

and denote by $\bar{A}_{k}$ its complement. Let $n \geq 1$ and remind that $v_{n}^{\varepsilon^{2}}=v_{n}+\left\lfloor n^{\varepsilon^{2}}\right\rfloor>$ $\left\lfloor n^{\varepsilon^{2}}\right\rfloor$. With the previous notation, we write

$$
\begin{aligned}
\mathbb{P}_{x}\left(\tau_{y}\right. & >n) \\
& =\mathbb{P}_{x}\left(\tau_{y}>n, v_{n}^{\varepsilon^{2}}>\left\lfloor n^{1-\varepsilon}\right\rfloor\right)
\end{aligned}
$$


(9.19)

$$
+\sum_{k=\left\lfloor n^{\varepsilon^{2}}\right\rfloor+1}^{\left\lfloor n^{1-\varepsilon}\right\rfloor} \int_{\mathbb{X} \times \mathbb{R}} \mathbb{P}_{x^{\prime}}\left(\tau_{y^{\prime}}>n-k, \bar{A}_{n-k}\right) \mathbb{P}_{x}\left(X_{k} \in \mathrm{d} x^{\prime}, y+S_{k} \in \mathrm{d} y^{\prime},\right.
$$

$$
\begin{aligned}
& \left.\tau_{y}>k, v_{n}^{\varepsilon^{2}}=k\right) \\
& =: J_{1} \\
& +\sum_{k=\left\lfloor n^{\varepsilon^{2}}\right\rfloor+1}^{\left\lfloor n^{1-\varepsilon}\right\rfloor} \int_{\mathbb{X} \times \mathbb{R}} \mathbb{P}_{x^{\prime}}\left(\tau_{y^{\prime}}>n-k, A_{n-k}\right) \mathbb{P}_{x}\left(X_{k} \in \mathrm{d} x^{\prime}, y+S_{k} \in \mathrm{d} y^{\prime},\right. \\
& \left.\tau_{y}>k, v_{n}^{\varepsilon^{2}}=k\right) . \\
& =: J_{2}
\end{aligned}
$$
have

Bound of $J_{1}$. Since $n-k \geq c_{\varepsilon} n$, for any $k \leq\left\lfloor n^{1-\varepsilon}\right\rfloor$, by Proposition 4.3, we

$$
\mathbb{P}_{x^{\prime}}\left(\tau_{y^{\prime}}>n-k, \bar{A}_{n-k}\right) \leq \mathbb{P}_{x^{\prime}}\left(\bar{A}_{n-k}\right) \leq \frac{c_{\varepsilon}\left(1+N\left(x^{\prime}\right)\right)}{n^{2 \varepsilon}} .
$$

So, using the fact that $n^{1 / 2-\varepsilon} \leq z+M_{v_{n}}$ and Lemma 9.1,

$$
\begin{aligned}
J_{1} & \leq \frac{c_{\varepsilon}}{n^{2 \varepsilon}} \mathbb{E}_{x}\left(1+\mathrm{e}^{-c n^{\varepsilon^{2}}} N\left(X_{v_{n}}\right) ; \tau_{y}>v_{n}, v_{n} \leq\left\lfloor n^{1-\varepsilon}\right\rfloor\right) \\
& \leq \frac{c_{\varepsilon}}{n^{1 / 2+\varepsilon}} E_{1}+c_{\varepsilon} \mathrm{e}^{-c_{\varepsilon} n^{\varepsilon^{2}}}(1+N(x)) \\
& \leq \frac{c_{\varepsilon}(1+\max (y, 0)+N(x))}{n^{1 / 2+\varepsilon}} .
\end{aligned}
$$

Bound of $J_{2}$. We split $J_{2}$ into two terms:

$$
\begin{aligned}
J_{2}= & \sum_{k=\left\lfloor n^{\varepsilon^{2}}\right\rfloor+1}^{\left\lfloor n^{1-\varepsilon}\right\rfloor} \int_{\mathbb{X} \times \mathbb{R}} \mathbb{P}_{x^{\prime}}\left(\tau_{y^{\prime}}>n-k, A_{n-k}\right) \\
& \underbrace{\times \mathbb{P}_{x}\left(X_{k} \in \mathrm{d} x^{\prime}, y+S_{k} \in \mathrm{d} y^{\prime}, y+S_{k}>n^{1 / 2-\varepsilon / 8}, \tau_{y}>k, v_{n}^{\varepsilon^{2}}=k\right)}_{=: J_{3}}
\end{aligned}
$$

$$
\begin{aligned}
& +\sum_{k=\left\lfloor n^{\varepsilon^{2}}\right\rfloor+1}^{\left\lfloor n^{1-\varepsilon}\right\rfloor} \int_{\mathbb{X} \times \mathbb{R}} \mathbb{P}_{x^{\prime}}\left(\tau_{y^{\prime}}>n-k, A_{n-k}\right) \\
& \underbrace{\times \mathbb{P}_{x}\left(X_{k} \in \mathrm{d} x^{\prime}, y+S_{k} \in \mathrm{d} y^{\prime}, y+S_{k} \leq n^{1 / 2-\varepsilon / 8}, \tau_{y}>k, v_{n}^{\varepsilon^{2}}=k\right)}_{=: J_{4}} .
\end{aligned}
$$

Bound of $J_{3}$. With $y_{+}^{\prime}=y^{\prime}+(n-k)^{1 / 2-2 \varepsilon}$, we have

$$
\mathbb{P}_{x^{\prime}}\left(\tau_{y^{\prime}}>n-k, A_{n-k}\right) \leq \mathbb{P}_{x^{\prime}}\left(\tau_{y_{+}^{\prime}}^{b m}>n-k\right) \text {, }
$$


where $\tau_{y}^{b m}$ is defined in (4.1). By point 1 of Lemma 4.2 and Lemma 9.5,

$$
\begin{aligned}
J_{3} \leq & \frac{c_{\varepsilon}}{\sqrt{n}} \mathbb{E}_{x}\left(y+S_{v_{n}^{\varepsilon^{2}}}+n^{1 / 2-2 \varepsilon}\right. \\
& \left.y+S_{v_{n}^{\varepsilon^{2}}}>n^{1 / 2-\varepsilon / 8}, \tau_{y}>v_{n}^{\varepsilon^{2}}, v_{n}^{\varepsilon^{2}} \leq\left\lfloor n^{1-\varepsilon}\right\rfloor\right) \\
\leq & \frac{2 c_{\varepsilon}}{\sqrt{n}} F_{4} \\
\leq & c_{\varepsilon} \frac{\max (y, 0)+\left(1+y \mathbb{1}_{\left\{y>n^{1 / 2-2 \varepsilon\}}\right.}+N(x)\right)^{2}}{n^{1 / 2+\varepsilon / 2}} .
\end{aligned}
$$

Upper bound of $J_{4}$. For $y^{\prime} \leq n^{1 / 2-\varepsilon / 8}$ and any $k \leq\left\lfloor n^{1-\varepsilon}\right\rfloor$, it holds $y_{+}^{\prime} \leq$ $2 n^{1 / 2-\varepsilon / 8} \leq c_{\varepsilon}(n-k)^{1 / 2-\varepsilon / 8}$. Therefore, by (9.22) and the point 2 of Lemma 4.2 with $\theta_{m}=\bar{c}_{\varepsilon} m^{-\varepsilon / 8}$ and $m=n-k$, we have

$$
\begin{aligned}
J_{4} \leq & \sum_{k=\left\lfloor n^{\varepsilon^{2}}\right\rfloor+1}^{\left\lfloor n^{1-\varepsilon}\right\rfloor} \int_{\mathbb{X} \times \mathbb{R}} \frac{2\left(1+\theta_{n-k}^{2}\right)}{\sqrt{2 \pi(n-k)} \sigma} \mathbb{E}_{x}\left(y+S_{k}+(n-k)^{1 / 2-2 \varepsilon} ;\right. \\
& \left.y+S_{k} \leq n^{1 / 2-\varepsilon / 8}, \tau_{y}>k, v_{n}^{\varepsilon^{2}}=k\right) .
\end{aligned}
$$

Since $\frac{2\left(1+\theta_{n-k}^{2}\right)}{\sqrt{2 \pi(n-k)} \sigma} \leq \frac{2}{\sqrt{2 \pi n} \sigma}\left(1+\frac{c_{\varepsilon}}{n^{\varepsilon / 4}}\right)$ and $n^{1 / 2-\varepsilon} \leq z+M_{v_{n}}$, we get

$$
\begin{aligned}
J_{4} \leq & \frac{2}{\sqrt{2 \pi n} \sigma}\left(1+\frac{c_{\varepsilon}}{n^{\varepsilon / 4}}\right) \mathbb{E}_{x}\left(y+S_{\nu_{n}^{\varepsilon^{2}}}+n^{1 / 2-2 \varepsilon} ; y+S_{\nu_{n}^{\varepsilon^{2}}} \leq n^{1 / 2-\varepsilon / 8}\right. \\
& \left.\tau_{y}>v_{n}^{\varepsilon^{2}}, v_{n}^{\varepsilon^{2}} \leq\left\lfloor n^{1-\varepsilon}\right\rfloor\right) \\
\leq & \frac{2}{\sqrt{2 \pi n} \sigma}\left(1+\frac{c_{\varepsilon}}{n^{\varepsilon / 4}}\right) F_{2}+\frac{c_{\varepsilon}}{n^{1 / 2+\varepsilon}} E_{1} .
\end{aligned}
$$

By Lemmas 9.1, 9.5 and (7.5),

$$
J_{4} \leq \frac{2 V(x, y)}{\sqrt{2 \pi n} \sigma}+\frac{c_{\varepsilon}(1+\max (y, 0)+N(x))}{n^{1 / 2+\varepsilon / 8}} .
$$

Lower bound of $J_{4}$. With $y_{-}^{\prime}=y^{\prime}-(n-k)^{1 / 2-2 \varepsilon}$, we note that $\mathbb{P}_{x^{\prime}}\left(\tau_{y^{\prime}}>n-\right.$ $\left.k, A_{n-k}\right) \geq \mathbb{P}_{x^{\prime}}\left(\tau_{y_{-}^{\prime}}^{b m}>n-k\right)-\mathbb{P}_{x^{\prime}}\left(\bar{A}_{n-k}\right)$. Considering the event $\left\{y+S_{k}>(n-\right.$ $k)^{1 / 2-2 \varepsilon}$ and repeating the arguments used to bound $J_{1}$ [see (9.20)], we obtain

$$
\begin{aligned}
J_{4} \geq & \sum_{k=\left\lfloor n^{\varepsilon^{2}}\right\rfloor+1}^{\left\lfloor n^{1-\varepsilon}\right\rfloor} \int_{\mathbb{X} \times \mathbb{R}} \mathbb{P}_{x^{\prime}}\left(\tau_{y_{-}^{\prime}}^{b m}>n-k\right) \mathbb{P}_{x}\left(X_{k} \in \mathrm{d} x^{\prime}, y+S_{k} \in \mathrm{d} y^{\prime},\right. \\
& \left.y+S_{k} \leq n^{1 / 2-\varepsilon / 8}, y+S_{k}>(n-k)^{1 / 2-2 \varepsilon}, \tau_{y}>k, v_{n}^{\varepsilon^{2}}=k\right) \\
& -\frac{c_{\varepsilon}(1+\max (y, 0)+N(x))}{n^{1 / 2+\varepsilon}} .
\end{aligned}
$$


Using point 2 of Lemma 4.2 and Proposition 4.3,

$$
\begin{aligned}
J_{4} \geq & \frac{2}{\sqrt{2 \pi n} \sigma}\left(1-\frac{c_{\varepsilon}}{n^{\varepsilon / 4}}\right) \mathbb{E}_{x}\left(y+S_{v_{n}^{\varepsilon^{2}}}-\left(n-v_{n}^{\varepsilon^{2}}\right)^{1 / 2-2 \varepsilon} ;\right. \\
& \left.y+S_{v_{n}^{\varepsilon^{2}}}>\left(n-v_{n}^{\varepsilon^{2}}\right)^{1 / 2-2 \varepsilon}, y+S_{v_{n}^{\varepsilon^{2}}} \leq n^{1 / 2-\varepsilon / 8}, \tau_{y}>v_{n}^{\varepsilon^{2}}, v_{n}^{\varepsilon^{2}} \leq\left\lfloor n^{1-\varepsilon}\right\rfloor\right) \\
& -\frac{c_{\varepsilon}(1+\max (y, 0)+N(x))}{n^{1 / 2+\varepsilon}} \\
\geq & \frac{2}{\sqrt{2 \pi n} \sigma}\left(1-\frac{c_{\varepsilon}}{n^{\varepsilon / 4}}\right) F_{2}-\frac{c_{\varepsilon}}{\sqrt{n}} F_{4}-\frac{c_{\varepsilon}}{n^{1 / 2+\varepsilon}} E_{1}-\frac{c_{\varepsilon}(1+\max (y, 0)+N(x))}{n^{1 / 2+\varepsilon}} .
\end{aligned}
$$

By Lemmas 9.1, 9.5 and (7.5),

$$
J_{4} \geq \frac{2 V(x, y)}{\sqrt{2 \pi n} \sigma}-c_{\varepsilon} \frac{\max (y, 0)+\left(1+y \mathbb{1}_{\left\{y>n^{1 / 2-2 \varepsilon}\right\}}+N(x)\right)^{2}}{n^{1 / 2+\varepsilon / 8}} .
$$

Putting together (9.25), (9.24), (9.23) and (9.21),

$$
\left|J_{2}-\frac{2 V(x, y)}{\sqrt{2 \pi n} \sigma}\right| \leq c_{\varepsilon} \frac{\max (y, 0)+\left(1+y \mathbb{1}_{\left\{y>n^{1 / 2-2 \varepsilon}\right\}}+N(x)\right)^{2}}{n^{1 / 2+\varepsilon / 8}} .
$$

Taking into account (9.20), (9.19) and Lemma 6.3, we conclude that, for any $(x, y) \in \mathbb{X} \times \mathbb{R}$,

$$
\left|\mathbb{P}_{x}\left(\tau_{y}>n\right)-\frac{2 V(x, y)}{\sqrt{2 \pi n} \sigma}\right| \leq c_{\varepsilon} \frac{\max (y, 0)+\left(1+y \mathbb{1}_{\left\{y>n^{1 / 2-2 \varepsilon}\right\}}+N(x)\right)^{2}}{n^{1 / 2+\varepsilon / 8}}
$$

Taking the limit as $n \rightarrow+\infty$ in (9.26), we obtain point 1 of Theorem 2.3. Point 2 of Theorem 2.3 is an immediate consequence of points 2 and 4 of Proposition 8.8.

9.3. Proof of Theorem 2.4. Point 1 of Theorem 2.4 is exactly (9.26). In order to prove point 2 of Theorem 2.4, we shall first establish a bound for $\mathbb{P}_{x}\left(\tau_{y}>n\right)$ when $z=y+r(x) \geq n^{1 / 2-\varepsilon}, n \geq 1$. Set $m_{\varepsilon}=n-\left\lfloor n^{\varepsilon}\right\rfloor$. By the Markov property,

$$
\begin{aligned}
\mathbb{P}_{x}\left(\tau_{y}>n\right)= & \int_{\mathbb{X} \times \mathbb{R}} \mathbb{P}_{x^{\prime}}\left(\tau_{y^{\prime}}>m_{\varepsilon}\right) \\
& \times \mathbb{P}_{x}\left(X_{\left\lfloor n^{\varepsilon}\right\rfloor} \in \mathrm{d} x^{\prime}, y+S_{\left\lfloor n^{\varepsilon}\right\rfloor} \in \mathrm{d} y^{\prime}, \tau_{y}>\left\lfloor n^{\varepsilon}\right\rfloor\right) .
\end{aligned}
$$

For any $x^{\prime} \in \mathbb{X}$ and $y^{\prime}>0$, using $A_{m_{\varepsilon}}$ defined by (9.18), we have

$$
\mathbb{P}_{x^{\prime}}\left(\tau_{y^{\prime}}>m_{\varepsilon}\right) \leq \mathbb{P}_{x^{\prime}}\left(\tau_{y_{+}^{\prime}}^{b m}>m_{\varepsilon}\right)+\mathbb{P}_{x^{\prime}}\left(\bar{A}_{m_{\varepsilon}}\right)
$$

where $\tau_{y_{+}^{\prime}}^{b m}$ is defined by (4.1) and $y_{+}^{\prime}=y^{\prime}+m_{\varepsilon}^{1 / 2-2 \varepsilon}$. By point 1 of Lemma 4.2 and Proposition 4.3,

$$
\mathbb{P}_{x^{\prime}}\left(\tau_{y^{\prime}}>m_{\varepsilon}\right) \leq \frac{c y_{+}^{\prime}}{\sqrt{m_{\varepsilon}}}+\frac{c_{\varepsilon}}{m_{\varepsilon}^{2 \varepsilon}}\left(1+N\left(x^{\prime}\right)\right) \leq \frac{c_{\varepsilon} y^{\prime}}{\sqrt{n}}+\frac{c_{\varepsilon}}{n^{2 \varepsilon}}+\frac{c_{\varepsilon}}{n^{2 \varepsilon}} N\left(x^{\prime}\right) .
$$


Introducing this bound in (9.27), we get

$$
\mathbb{P}_{x}\left(\tau_{y}>n\right) \leq \frac{c_{\varepsilon}}{\sqrt{n}} \mathbb{E}_{x}\left(y+S_{\left\lfloor n^{\varepsilon}\right\rfloor}, \tau_{y}>\left\lfloor n^{\varepsilon}\right\rfloor\right)+\frac{c_{\varepsilon}}{n^{2 \varepsilon}}+\frac{c_{\varepsilon}}{n^{2 \varepsilon}} \mathbb{E}_{x}\left(N\left(X_{\left\lfloor n^{\varepsilon}\right\rfloor}\right)\right) .
$$

Using Corollary 6.5, the inequality (2.2) and the fact that $n^{1 / 2-\varepsilon} \leq z$, we find

$$
\mathbb{P}_{x}\left(\tau_{y}>n\right) \leq \frac{c_{\varepsilon}(z+N(x))}{\sqrt{n}}
$$

Now, for any $x \in \mathbb{X}, z \in \mathbb{R}$ and $y=z-r(x)$, using the Markov property, (9.28) and the fact that $\sqrt{n-v_{n}} \geq c_{\varepsilon} \sqrt{n}$ on the event $\left\{v_{n} \leq\left\lfloor n^{1-\varepsilon}\right\rfloor\right\}$, we have

$$
\begin{aligned}
\mathbb{P}_{x}\left(\tau_{y}>n\right) \leq & \frac{c_{\varepsilon}}{\sqrt{n}} \mathbb{E}_{x}\left(z+M_{v_{n}}+N\left(X_{v_{n}}\right) ; \tau_{y}>v_{n}, v_{n} \leq\left\lfloor n^{1-\varepsilon}\right\rfloor\right) \\
& +\mathbb{P}_{x}\left(\tau_{y}>n, v_{n}>\left\lfloor n^{1-\varepsilon}\right\rfloor\right) .
\end{aligned}
$$

Using Lemma 6.3 and the fact that $N\left(X_{v_{n}}\right) \leq z+M_{v_{n}}$ on the event $\left\{N\left(X_{v_{n}}\right) \leq\right.$ $\left.n^{1 / 2-\varepsilon}\right\}$, with $l=\left\lfloor n^{1 / 2-\varepsilon}\right\rfloor$, it holds

$$
\begin{aligned}
\mathbb{P}_{x}\left(\tau_{y}>n\right) \leq & \frac{c_{\varepsilon}}{\sqrt{n}} \mathbb{E}_{x}\left(\left(z+M_{v_{n}}\right)\left(1+\mathbb{1}_{\left\{N\left(X_{v_{n}}\right) \leq n^{1 / 2-\varepsilon}\right\}}\right) ; \tau_{y}>v_{n}, v_{n} \leq\left\lfloor n^{1-\varepsilon}\right\rfloor\right) \\
& +\frac{c_{\varepsilon}}{\sqrt{n}} \mathbb{E}_{x}\left(N_{l}\left(X_{v_{n}}\right) ; \tau_{y}>v_{n}, v_{n} \leq\left\lfloor n^{1-\varepsilon}\right\rfloor\right)+c_{\varepsilon} \mathrm{e}^{-c_{\varepsilon} n^{\varepsilon}}(1+N(x)) \\
\leq & \frac{2 c_{\varepsilon}}{\sqrt{n}} E_{1}+\frac{c_{\varepsilon}}{\sqrt{n}} \sum_{k=1}^{\left\lfloor n^{\varepsilon}\right\rfloor} \mathbb{E}_{x}\left(N_{l}\left(X_{k}\right)\right) \\
& +\frac{c_{\varepsilon}}{\sqrt{n}} \sum_{k=\left\lfloor n^{\varepsilon}\right\rfloor+1}^{\left\lfloor n^{1-\varepsilon}\right\rfloor} \mathbb{E}_{x}\left(N_{l}\left(X_{k}\right) ; \tau_{y}>k\right)+c_{\varepsilon} \mathrm{e}^{-c_{\varepsilon} n^{\varepsilon}}(1+N(x)) .
\end{aligned}
$$

By (2.3) and the Markov property,

$$
\begin{aligned}
\mathbb{P}_{x}\left(\tau_{y}>n\right) \leq & \frac{c_{\varepsilon}}{\sqrt{n}} E_{1}+\frac{c_{\varepsilon}}{\sqrt{n}}\left(\frac{c n^{\varepsilon}}{l^{1+\beta}}+(1+N(x))\right)+c_{\varepsilon} \mathrm{e}^{-c_{\varepsilon} n^{\varepsilon}}(1+N(x)) \\
& +\frac{c_{\varepsilon}}{\sqrt{n}} \sum_{j=1}^{\left\lfloor n^{1-\varepsilon}\right\rfloor-\left\lfloor n^{\varepsilon}\right\rfloor}\left[\frac{c}{l^{1+\beta}} \mathbb{P}_{x}\left(\tau_{y}>j\right)+c \mathrm{e}^{-c n^{\varepsilon}} \mathbb{E}_{x}\left(\left(1+N\left(X_{j}\right)\right)\right)\right] \\
\leq & \frac{c_{\varepsilon}}{\sqrt{n}} E_{1}+\frac{c_{\varepsilon}(1+N(x))}{\sqrt{n}}+\frac{c_{\varepsilon}}{\sqrt{n}} \frac{c}{l^{1+\beta}} \sum_{j=1}^{\left\lfloor n^{1-\varepsilon}\right\rfloor} \mathbb{P}_{x}\left(\tau_{y}>j\right) .
\end{aligned}
$$

Using Lemmas 9.1 and 9.2, we deduce point 2 of Theorem 2.4. 
10. Asymptotic behaviour of the conditioned Markov walk. In this section, we prove Theorem 2.5. The arguments are similar to those given in Section 9. We also keep the same notations. Assume that $(x, y) \in \mathbb{X} \times \mathbb{R}$ and let $t_{0}>0$ be a positive real. For any $t \in\left[0, t_{0}\right]$ and $n \geq 1$, we write

$$
\begin{aligned}
& \mathbb{P}_{x}\left(y+S_{n} \leq t \sqrt{n}, \tau_{y}>n\right) \\
& =\mathbb{P}_{x}\left(y+S_{n} \leq t \sqrt{n}, \tau_{y}>n, v_{n}^{\varepsilon^{2}}>\left\lfloor n^{1-\varepsilon}\right\rfloor\right) \\
& +\sum_{k=\left\lfloor n^{\varepsilon^{2}}\right\rfloor+1}^{\left\lfloor n^{1-\varepsilon}\right\rfloor} \int_{\mathbb{X} \times \mathbb{R}} \mathbb{P}_{x^{\prime}}\left(y^{\prime}+S_{n-k} \leq t \sqrt{n}, \tau_{y^{\prime}}>n-k, \bar{A}_{n-k}\right) \\
& \underbrace{\times \mathbb{P}_{x}\left(X_{k} \in \mathrm{d} x^{\prime}, y+S_{k} \in \mathrm{d} y^{\prime}, \tau_{y}>k, v_{n}^{\varepsilon^{2}}=k\right)}_{=: L_{1}} \\
& +\sum_{k=\left\lfloor n^{\varepsilon^{2}}\right\rfloor+1}^{\left\lfloor n^{1-\varepsilon}\right\rfloor} \int_{\mathbb{X} \times \mathbb{R}} \mathbb{P}_{x^{\prime}}\left(y^{\prime}+S_{n-k} \leq t \sqrt{n}, \tau_{y^{\prime}}>n-k, A_{n-k}\right) \\
& \underbrace{\times \mathbb{P}_{x}\left(X_{k} \in \mathrm{d} x^{\prime}, y+S_{k} \in \mathrm{d} y^{\prime}, \tau_{y}>k, v_{n}^{\varepsilon^{2}}=k\right)}_{=: L_{2}} .
\end{aligned}
$$

Bound of $L_{1}$. With $J_{1}$ defined in (9.19) and with the bound (9.20), we have,

$$
L_{1} \leq J_{1} \leq \frac{c_{\varepsilon}(1+\max (y, 0)+N(x))}{n^{1 / 2+\varepsilon}} .
$$

Bound of $L_{2}$. According to whether $y+S_{k} \leq n^{1 / 2-\varepsilon / 8}$ or not, we write

$$
\begin{aligned}
L_{2}= & \sum_{k=\left\lfloor n^{\varepsilon^{2}}\right\rfloor+1}^{\left\lfloor n^{1-\varepsilon}\right\rfloor} \int_{\mathbb{X} \times \mathbb{R}} \mathbb{P}_{x^{\prime}}\left(y^{\prime}+S_{n-k} \leq t \sqrt{n}, \tau_{y^{\prime}}>n-k, A_{n-k}\right) \\
& \underbrace{\times \mathbb{P}_{x}\left(X_{k} \in \mathrm{d} x^{\prime}, y+S_{k} \in \mathrm{d} y^{\prime}, y+S_{k}>n^{1 / 2-\varepsilon / 8}, \tau_{y}>k, v_{n}^{\varepsilon^{2}}=k\right)}_{=: L_{3}}
\end{aligned}
$$

$$
\begin{aligned}
& +\sum_{k=\left\lfloor n^{\varepsilon^{2}}\right\rfloor+1}^{\left\lfloor n^{1-\varepsilon}\right\rfloor} \int_{\mathbb{X} \times \mathbb{R}} \mathbb{P}_{x^{\prime}}\left(y^{\prime}+S_{n-k} \leq t \sqrt{n}, \tau_{y^{\prime}}>n-k, A_{n-k}\right) \\
& \underbrace{\times \mathbb{P}_{x}\left(X_{k} \in \mathrm{d} x^{\prime}, y+S_{k} \in \mathrm{d} y^{\prime}, y+S_{k} \leq n^{1 / 2-\varepsilon / 8}, \tau_{y}>k, v_{n}^{\varepsilon^{2}}=k\right)}_{=: L_{4}} .
\end{aligned}
$$

Bound of $L_{3}$. With $J_{3}$ defined in (9.21) and with the bound (9.23), we have

$$
L_{3} \leq J_{3} \leq c_{\varepsilon} \frac{\max (y, 0)+\left(1+y \mathbb{1}_{\left\{y>n^{1 / 2-2 \varepsilon}\right\}}+N(x)\right)^{2}}{n^{1 / 2+\varepsilon / 2}} .
$$


Bound of $L_{4}$. We start with the upper bound. Set $y_{+}^{\prime}=y^{\prime}+(n-k)^{1 / 2-2 \varepsilon}$ and $t_{+}=t+\frac{2}{n^{2 \varepsilon}}$. Note that on the event $\left\{y^{\prime}+S_{n-k} \leq t \sqrt{n}, \tau_{y^{\prime}}>n-k, A_{n-k}\right\}$ we have $y_{+}^{\prime}+\sigma B_{n-k} \leq t_{+} \sqrt{n}$ and $\tau_{y_{+}^{\prime}}^{b m}>n-k$. Therefore, by Lemma 4.1,

$$
\begin{aligned}
\mathbb{P}_{x^{\prime}}\left(y^{\prime}+S_{n-k} \leq t \sqrt{n}, \tau_{y^{\prime}}>n-k, A_{n-k}\right) \\
\quad \leq \frac{2}{\sqrt{2 \pi}} \int_{0}^{\frac{t_{+} \sqrt{n}}{\sigma \sqrt{n-k}}} \mathrm{e}^{-s^{2} / 2} \operatorname{sh}\left(s \frac{y_{+}^{\prime}}{\sqrt{n-k} \sigma}\right) \mathrm{d} s .
\end{aligned}
$$

We shall use the following bounds:

$$
\begin{array}{ll}
\operatorname{sh}(u) \leq u\left(1+\frac{u^{2}}{6} \operatorname{ch}(u)\right), & \text { for } u \geq 0, \\
\frac{y_{+}^{\prime}}{\sigma \sqrt{n-k}} \leq \frac{y_{+}^{\prime}}{\sigma \sqrt{n}}\left(1+\frac{c_{\varepsilon}}{n^{\varepsilon}}\right) \leq \frac{c_{\varepsilon}}{n^{\varepsilon / 8}}, & \text { for } y^{\prime} \leq n^{1 / 2-\varepsilon / 8} \text { and } k \leq\left\lfloor n^{1-\varepsilon}\right\rfloor, \\
\frac{t_{+} \sqrt{n}}{\sigma \sqrt{n-k}} \leq \frac{t}{\sigma}+\frac{c_{\varepsilon, t_{0}}}{n^{\varepsilon}} \leq c_{\varepsilon, t_{0}}, & \text { for } k \leq\left\lfloor n^{1-\varepsilon}\right\rfloor .
\end{array}
$$

Consequently,

$$
\begin{aligned}
\mathbb{P}_{x^{\prime}}\left(y^{\prime}\right. & \left.+S_{n-k} \leq t \sqrt{n}, \tau_{y^{\prime}}>n-k, A_{n-k}\right) \\
& \leq \frac{2 y_{+}^{\prime}}{\sqrt{2 \pi n} \sigma}\left(1+\frac{c_{\varepsilon}}{n^{\varepsilon}}\right) \int_{0}^{\frac{t+\sqrt{n}}{\sigma \sqrt{n-k}}} s \mathrm{e}^{-s^{2} / 2}\left(1+\frac{c_{\varepsilon} s^{2}}{n^{\varepsilon / 4}} \mathrm{ch}\left(c_{\varepsilon} s\right)\right) \mathrm{d} s \\
& \leq \frac{2 y_{+}^{\prime}}{\sqrt{2 \pi n} \sigma}\left(1+\frac{c_{\varepsilon}}{n^{\varepsilon}}\right)\left(1+\frac{c_{\varepsilon, t_{0}}}{n^{\varepsilon / 4}}\right)\left(\int_{0}^{\frac{t}{\sigma}} s \mathrm{e}^{-s^{2} / 2} \mathrm{~d} s+\int_{\frac{t}{\sigma}}^{\frac{t_{+} \sqrt{n}}{\sigma \sqrt{n-k}}} \mathrm{e}^{-s^{2} / 2} \mathrm{~d} s\right) \\
& \leq \frac{2 y_{+}^{\prime}}{\sqrt{2 \pi n} \sigma}\left(1+\frac{c_{\varepsilon, t_{0}}}{n^{\varepsilon / 4}}\right)\left(1-\mathrm{e}^{-\frac{t^{2}}{2 \sigma^{2}}}+\frac{c_{\varepsilon, t_{0}}}{n^{\varepsilon}}\right) .
\end{aligned}
$$

This implies the upper bound (with $F_{2}$ and $E_{1}$ from Lemmas 9.5 and 9.1, respectively)

$$
\begin{aligned}
L_{4} & \leq \frac{2}{\sqrt{2 \pi n} \sigma}\left(1+\frac{c_{\varepsilon, t_{0}}}{n^{\varepsilon / 4}}\right)\left(1-\mathrm{e}^{-\frac{t^{2}}{2 \sigma^{2}}}+\frac{c_{\varepsilon, t_{0}}}{n^{\varepsilon}}\right) F_{2}+\frac{c_{\varepsilon, t_{0}}}{n^{1 / 2+\varepsilon}} E_{1} \\
& \leq \frac{2 V(x, y)}{\sqrt{2 \pi n} \sigma}\left(1-\mathrm{e}^{-\frac{t^{2}}{2 \sigma^{2}}}\right)+\frac{c_{\varepsilon, t_{0}}(1+\max (y, 0)+N(x))}{n^{1 / 2+\varepsilon / 8}} .
\end{aligned}
$$

The proof of the lower bound of $L_{4}$, being similar, is left to the reader:

$$
L_{4} \geq \frac{2 V(x, y)}{\sqrt{2 \pi n} \sigma}\left(1-\mathrm{e}^{-\frac{t^{2}}{2 \sigma^{2}}}\right)-c_{\varepsilon, t_{0}} \frac{\max (y, 0)+\left(1+y \mathbb{1}_{\left\{y>n^{1 / 2-2 \varepsilon}\right\}}+N(x)\right)^{2}}{n^{1 / 2+\varepsilon / 8}} .
$$

Combining the upper and the lower bounds of $L_{4}$ and (10.4) with (10.3) we obtain an asymptotic development of $L_{2}$. Implementing this development and the bound 
(10.2) into (10.1) and using Lemma 6.3, we conclude that

$$
\begin{gathered}
\left|\mathbb{P}_{x}\left(y+S_{n} \leq t \sqrt{n}, \tau_{y}>n\right)-\frac{2 V(x, y)}{\sqrt{2 \pi n} \sigma}\left(1-\mathrm{e}^{-\frac{t^{2}}{2 \sigma^{2}}}\right)\right| \\
\leq c_{\varepsilon, t_{0}} \frac{\max (y, 0)+\left(1+y \mathbb{1}_{\left\{y>n^{1 / 2-2 \varepsilon}\right\}}+N(x)\right)^{2}}{n^{1 / 2+\varepsilon / 8}} .
\end{gathered}
$$

Using the asymptotic of $\mathbb{P}_{x}\left(\tau_{y}>n\right)$ provided by Theorem 2.3 finishes the proof of Theorem 2.5.

\section{APPENDIX A: PROOFS FOR AFFINE RANDOM WALKS IN $\mathbb{R}^{d}$}

In this section, we prove Proposition 3.2. For this we verify that Hypotheses M1-M5 hold true on an appropriate Banach space which we proceed to introduce. Let $\delta>0$ be the constant from Hypothesis 3.1. Denote by $\mathscr{C}\left(\mathbb{R}^{d}\right)$ the space of continuous complex valued functions on $\mathbb{R}^{d}$. Let $\varepsilon$ and $\theta$ be two positive numbers satisfying

$$
1+\varepsilon<\theta<2<2+2 \varepsilon<2+2 \delta .
$$

For any function $h \in \mathscr{C}\left(\mathbb{R}^{d}\right)$ introduce the norm $\|h\|_{\theta, \varepsilon}=|h|_{\theta}+[h]_{\varepsilon}$, where

$$
|h|_{\theta}=\sup _{x \in \mathbb{R}^{d}} \frac{|h(x)|}{(1+|x|)^{\theta}}, \quad[h]_{\varepsilon}=\sup _{x \neq y} \frac{|h(x)-h(y)|}{|x-y|^{\varepsilon}(1+|x|)(1+|y|)}
$$

and consider the Banach space

$$
\mathscr{B}:=\mathscr{L}_{\theta, \varepsilon}=\left\{h \in \mathscr{C}\left(\mathbb{R}^{d}\right):\|h\|_{\theta, \varepsilon}<+\infty\right\} .
$$

Proof of M1. Conditions 1, 2 and 3 of M1 can be easily verified under point 1 of Hypothesis 3.1 and the fact that $\theta<2+2 \delta$ and $\left\|\delta_{x}\right\|_{\mathscr{B}^{\prime}} \leq(1+|x|)^{\theta}$, for any $x \in \mathbb{R}^{d}$.

We verify point 4 of Hypothesis M1. For any $(x, y) \in \mathbb{R}^{d} \times \mathbb{R}^{d}$ and $t \in \mathbb{R}$, we have $\left|\mathrm{e}^{i t f(x)}-\mathrm{e}^{i t f(y)}\right| \leq|t||f(x)-f(y)| \leq|t||u||x-y|$ and $\left|\mathrm{e}^{i t f(x)}-\mathrm{e}^{i t f(y)}\right| \leq 2$. Therefore, we write

$$
\left|\mathrm{e}^{i t f(x)}-\mathrm{e}^{i t f(y)}\right| \leq 2^{1-\varepsilon}|t|^{\varepsilon}|u|^{\varepsilon}|x-y|^{\varepsilon} .
$$

Supposing that $|x| \leq|y|$, we obtain, for any $h \in \mathscr{L}_{\theta, \varepsilon}$,

$$
\left|\mathrm{e}^{i t f(x)} h(x)-\mathrm{e}^{i t f(y)} h(y)\right| \leq\left|\mathrm{e}^{i t f(x)}-\mathrm{e}^{i t f(y)}\right||h|_{\theta}(1+|x|)^{\theta}+|h(x)-h(y)| .
$$

Since $\theta<2$, we deduce that $\left[\mathrm{e}^{i t f} h-\mathrm{e}^{i t f} h\right]_{\varepsilon} \leq 2^{1-\varepsilon}|t|^{\varepsilon}|u|^{\varepsilon}|h|_{\theta}+[h]_{\varepsilon}$. Consequently, $\left\|\mathrm{e}^{i t f} h\right\|_{\theta, \varepsilon} \leq\left(1+2^{1-\varepsilon}|t|^{\varepsilon}|u|^{\varepsilon}\right)\|h\|_{\theta, \varepsilon}$ and the point 4 is verified.

Proof of M2 and M3. We shall verify that the conditions of the theorem of Ionescu Tulcea and Marinescu are satisfied (see [28] and [25]). We start by establishing two lemmas.

LemMA A.1. Assume Hypothesis 3.1. 
1. There exists a constant $c>0$ such that, for any $t \in \mathbb{R}, n \geq 1$, and $h \in \mathscr{L}_{\theta, \varepsilon}$,

$$
\left|\mathbf{P}_{t}^{n} h\right|_{\theta} \leq c|h|_{\theta} .
$$

2. There exist constants $c_{1}, c_{2}$ and $\rho<1$ such that, for any $n \geq 1, h \in \mathscr{L}_{\theta, \varepsilon}$ and $t \in \mathbb{R}$,

$$
\left[\mathbf{P}_{t}^{n} h\right]_{\varepsilon} \leq c_{1} \rho^{n}[h]_{\varepsilon}+c_{2}|t|^{\varepsilon}|h|_{\theta} .
$$

3. For any $t \in \mathbb{R}$, the operator $\mathbf{P}_{t}$ is compact from $\left(\mathscr{B},\|\cdot\|_{\theta, \varepsilon}\right)$ to $\left(\mathscr{C}\left(\mathbb{R}^{d}\right),|\cdot|_{\theta}\right)$.

ProOF. Claim 1. For any $x \in \mathbb{R}^{d}$,

$$
\left|\mathbf{P}_{t}^{n} h(x)\right|=\left|\mathbb{E}_{x}\left(\mathrm{e}^{i t S_{n}} h\left(X_{n}\right)\right)\right| \leq 3^{\theta}|h|_{\theta}\left(1+\mathbb{E}\left(\left\|\Pi_{n}\right\|^{\theta}\right)|x|^{\theta}+\mathbb{E}\left(\left|X_{n}^{0}\right|^{\theta}\right)\right),
$$

with $\Pi_{n}=A_{n} A_{n-1} \cdots A_{1}$ and $X_{n}^{0}=g_{n} \cdots g_{1} \cdot 0=\sum_{k=1}^{n} A_{n} \cdots A_{k+1} B_{k}$. By point 1 of Hypothesis 3.1, there exist $c(\delta)>0$ and $0<\rho(\delta)<1$ such that, for any $n \geq 1$,

$$
\mathbb{E}^{\frac{2+2 \delta}{\theta}}\left(\left\|\Pi_{n}\right\|^{\theta}\right) \leq \mathbb{E}\left(\left\|\Pi_{n}\right\|^{2+2 \delta}\right) \leq c(\delta) \rho(\delta)^{n} \underset{n \rightarrow+\infty}{\longrightarrow} 0,
$$

from which it follows that

$$
\mathbb{E}\left(\left|X_{n}^{0}\right|^{\theta}\right) \leq\left(\sum_{k=1}^{n} \mathbb{E}^{1 / \theta}\left(\left\|\Pi_{n}\right\|^{\theta}\right) \mathbb{E}^{1 / \theta}\left(\left|B_{1}\right|^{\theta}\right)\right)^{\theta}<+\infty .
$$

This proves claim 1 .

Proof of claim 2. For any $x \neq y \in \mathbb{R}^{d}$, with $|x| \leq|y|$, we have

$$
\begin{aligned}
\mid \mathbf{P}_{t}^{n} h(x) & -\mathbf{P}_{t}^{n} h(y) \mid \\
\leq & \mathbb{E}\left(2^{1-\varepsilon}|t|^{\varepsilon}|u|^{\varepsilon}\left(\sum_{k=1}^{n}\left\|\Pi_{k}\right\|\right)^{\varepsilon}|x-y|^{\varepsilon}|h|_{\theta}\left(1+\left\|\Pi_{n}\right\||x|+\left|X_{n}^{0}\right|\right)^{\theta}\right) \\
& +\mathbb{E}\left([h]_{\varepsilon}\left\|\Pi_{n}\right\|^{\varepsilon}|x-y|^{\varepsilon}\left(1+\left\|\Pi_{n}\right\||x|+\left|X_{n}^{0}\right|\right)\left(1+\left\|\Pi_{n}\right\||y|+\left|X_{n}^{0}\right|\right)\right) .
\end{aligned}
$$

Since $\theta<2$, we obtain that

$$
\left[\mathbf{P}_{t}^{n} h\right]_{\varepsilon} \leq 2^{1-\varepsilon}|t|^{\varepsilon}|u|^{\varepsilon} C_{2}(n)|h|_{\theta}+C_{1}(n)[h]_{\varepsilon},
$$

where

$$
C_{1}(n)=\mathbb{E}\left(\left\|\Pi_{n}\right\|^{\varepsilon}\left(1+\left\|\Pi_{n}\right\|+\left|X_{n}^{0}\right|\right)^{2}\right)
$$

and

$$
C_{2}(n)=\mathbb{E}\left(\left(\sum_{k=1}^{n}\left\|\Pi_{k}\right\|\right)^{\varepsilon}\left(1+\left\|\Pi_{n}\right\|+\left|X_{n}^{0}\right|\right)^{\theta}\right)
$$


Since $2+2 \varepsilon<2+2 \delta=p$, by the Hölder inequality,

$$
\begin{aligned}
C_{1}(n) & \leq \mathbb{E}^{\frac{\varepsilon}{1+\varepsilon}}\left(\left\|\Pi_{n}\right\|^{1+\varepsilon}\right) \mathbb{E}^{\frac{1}{1+\varepsilon}}\left(\left(1+\left\|\Pi_{n}\right\|+\left|X_{n}^{0}\right|\right)^{2+2 \varepsilon}\right) \\
& \leq c(\delta)^{\frac{\varepsilon}{p}} \rho(\delta)^{\frac{n \varepsilon}{p}} 3^{2}\left(1+c(\delta)^{\frac{2}{p}}+\left(\frac{c(\delta)^{\frac{1}{p}} \mathbb{E}^{\frac{1}{p}}\left(\left|B_{1}\right|^{p}\right)}{1-\rho(\delta)^{\frac{1}{p}}}\right)^{2}\right),
\end{aligned}
$$

which shows that $C_{1}(n)$ converges exponentially fast to 0 . In the same way, taking into account that $\theta<2$ we show that $C_{2}(n)$ is bounded:

$$
\begin{aligned}
C_{2}(n) & \leq\left(\sum_{k=1}^{n} \mathbb{E}^{\frac{1}{1+\varepsilon}}\left(\left\|\Pi_{k}\right\|^{1+\varepsilon}\right)\right)^{\varepsilon} \mathbb{E}^{\frac{1}{1+\varepsilon}}\left(\left(1+\left\|\Pi_{n}\right\|+\left|X_{n}^{0}\right|\right)^{2+2 \varepsilon}\right) \\
& \leq\left(\frac{c(\delta)^{\frac{1}{p}}}{1-\rho(\delta)^{\frac{1}{p}}}\right)^{\varepsilon} 3^{2}\left(1+c(\delta)^{\frac{2}{p}}+\left(\frac{c(\delta)^{\frac{1}{p}} \mathbb{E}^{\frac{1}{p}}\left(\left|B_{1}\right|^{p}\right)}{1-\rho(\delta)^{\frac{1}{p}}}\right)^{2}\right) .
\end{aligned}
$$

Proof of claim 3. Let $B$ be a bounded subset of $\mathscr{B},\left(h_{n}\right)_{n \geq 0}$ be a sequence in $B$ and $K$ be a compact of $\mathbb{R}^{d}$. Using claim 1, it follows that, for any $x \in K$ and $n \geq 0$,

$$
\left|\mathbf{P}_{t} h_{n}(x)\right| \leq c\left|h_{n}\right|_{\theta}(1+|x|)^{\theta} \leq c_{K},
$$

which implies that the set $\mathscr{A}=\left\{\mathbf{P}_{t} h_{n}: n \geq 0\right\}$ is uniformly bounded in $\left(\mathscr{C}(K),|\cdot|_{\infty}\right)$, where $|\cdot|_{\infty}$ is the supremum norm. By claims 1 and 2 , we have that, for any $x, y \in K$ and $n \geq 0$,

$$
\left|\mathbf{P}_{t} h_{n}(x)-\mathbf{P}_{t} h_{n}(y)\right| \leq\left[\mathbf{P}_{t} h_{n}\right]_{\varepsilon}|x-y|^{\varepsilon}(1+|x|)^{\theta}(1+|y|)^{\theta} \leq c_{K}\left\|h_{n}\right\|_{\mathscr{B}}|x-y|^{\varepsilon}
$$

and, thereby, the set $\mathscr{A}$ is uniformly equicontinuous. By the theorem of ArzelàAscoli, we conclude that $\mathscr{A}$ is relatively compact in $\left(\mathscr{C}(K),|\cdot|_{\infty}\right)$. Using a diagonal extraction, we deduce that there exist a subsequence $\left(n_{k}\right)_{k \geq 1}$ and a function $\varphi \in \mathscr{C}\left(\mathbb{R}^{d}\right)$ such that, for any compact $K \subset \mathbb{R}^{d}$,

$$
\sup _{x \in K}\left|P_{t} h_{n_{k}}(x)-\varphi(x)\right| \underset{n \rightarrow+\infty}{\longrightarrow} 0 .
$$

Moreover, by claims 1 and 2, for any $n \geq 1$ and $x \in \mathbb{R}^{d}$,

$$
\left|P_{t} h_{n}(x)\right| \leq\left|P_{t} h_{n}(0)\right|+\left[P_{t} h_{n}\right]_{\varepsilon}|x|^{\varepsilon}(1+|x|) \leq c\left|h_{n}\right|_{\theta}+c\left\|h_{n}\right\|_{\mathscr{B}}|x|^{\varepsilon}(1+|x|) .
$$

Since $B$ is bounded, we have $\left|P_{t} h_{n}(x)\right| \leq c(1+|x|)^{1+\varepsilon}$, for any $x \in \mathbb{R}^{d}$, as well as $\varphi(x) \leq c(1+|x|)^{1+\varepsilon}$, for any $x \in \mathbb{R}^{d}$. Consequently, for any $k \geq 1$ and $A>0$,

$$
\sup _{x \in \mathbb{R}^{d}} \frac{\left|P_{t} h_{n_{k}}(x)-\varphi(x)\right|}{(1+|x|)^{\theta}} \leq \sup _{|x| \leq A}\left|P_{t} h_{n_{k}}(x)-\varphi(x)\right|+2 c \sup _{|x|>A} \frac{(1+|x|)^{1+\varepsilon}}{(1+|x|)^{\theta}} .
$$


Taking the limit as $k \rightarrow+\infty$ and then the limit as $A \rightarrow+\infty$, we conclude that

$$
\lim _{k \rightarrow+\infty}\left|P_{t} h_{n_{k}}-\varphi\right|_{\theta}=0 .
$$

Lemma A.2. Assume Hypothesis 3.1. 1. The operator $\mathbf{P}$ has a unique invariant probability $\boldsymbol{v}$ which coincides with the distribution of the $\mathbb{P}$-a.s. convergent series $Z:=\sum_{k=1}^{+\infty} A_{1} \cdots A_{k-1} B_{k}$. Moreover, the unique eigenvalue of modulus 1 of the operator $\mathbf{P}$ on $\mathscr{B}$ is 1 and the associated eigenspace is generated by the function $e: x \mapsto 1$.

2. Let $t \in \mathbb{R}^{*}$. If $h \in \mathscr{B}$ and $z \in \mathbb{C}$ of modulus 1 are such that

$$
\mathbf{P}_{t} h(x)=z h(x), \quad x \in \operatorname{supp}(\boldsymbol{v}),
$$

then $h=0$ on $\operatorname{supp}(\boldsymbol{v})$.

ProOF. We proceed as in Guivarc' $h$ and Le Page [22] and Buraczewski, Damek and Guivarc'h [5]. For any $g=(A, B) \in \operatorname{GL}(d, \mathbb{R}) \times \mathbb{R}^{d}$ and $x \in \mathbb{R}^{d}$, we set $g \cdot x=A x+B$.

Proof of claim 1. Since $k(\delta)<1$, the series $\sum_{k} \mathbb{E}^{\frac{1}{2+2 \delta}}\left(\left|A_{1} \cdots A_{k-1} B_{k}\right|^{2+2 \delta}\right)$ converges and so the sequence $g_{1} \cdots g_{n} \cdot x=A_{1} \cdots A_{n} x+\sum_{k=1}^{n} A_{1} \cdots A_{k-1} B_{k}$ converges almost surely to $Z=\sum_{k=1}^{+\infty} A_{1} \cdots A_{k-1} B_{k}$ as $n \rightarrow+\infty$. Therefore, for any $\varphi \in \mathscr{B}$, the sequence $\varphi\left(g_{1} \cdots g_{n} \cdot x\right)$ converges to $\varphi(Z)$ almost surely as $n \rightarrow+\infty$. Moreover, since $|\varphi(x)| \leq|\varphi|_{\theta}(1+|x|)^{\theta}$ and $\theta<2+2 \delta$, the sequence $\left(\varphi\left(g_{1} \cdots g_{n} \cdot x\right)\right)_{n \geq 1}$ is uniformly integrable. So $\mathbf{P}^{n} \varphi(x)$ converges to $\mathbb{E}(\varphi(Z))$ as $n \rightarrow+\infty$. This proves that the distribution $\boldsymbol{v}$ of $Z$ is the only invariant probability of $\mathbf{P}$.

Fix $z \in \mathbb{C}$ such that $|z|=1$ and let $h \neq 0$ belonging to $\mathscr{B}$ be an eigenfunction of $\mathbf{P}$, so that $\mathbf{P} h=z h$. From the previous argument, it follows that, for any $x \in \mathbb{R}^{d}$,

$$
z^{n} h(x)=\mathbf{P}^{n} h(x) \underset{n \rightarrow+\infty}{\longrightarrow} \mathbf{v}(h) .
$$

Since there exists $x \in \mathbb{R}^{d}$ such that $h(x) \neq 0$, the sequence $\left(z^{n}\right)_{n \geq 1}$ should be convergent which is possible only if $z=1$. From this, we deduce that for any $x \in \mathbb{R}^{d}, h(x)=\mathbb{E}(h(Z))$ which implies that $h$ is constant.

Proof of claim 2. Our argument is by contradiction. Let $t \in \mathbb{R}^{*}, h \in \mathscr{B}$ and $z \in \mathbb{C}$ of modulus 1 be such that $\mathbf{P}_{t} h(x)=z h(x)$, for any $x \in \operatorname{supp}(\boldsymbol{v})$ and suppose that there exists $x_{0} \in \operatorname{supp}(\boldsymbol{v})$ such that $h\left(x_{0}\right) \neq 0$.

First, we establish that $|h|$ is constant on the support of the distribution $\boldsymbol{v}$. Since $\boldsymbol{v}$ is $\boldsymbol{\mu}$-invariant, for any $(g, x) \in \operatorname{supp}(\boldsymbol{\mu}) \times \operatorname{supp}(\boldsymbol{v})$ we have $g \cdot x \in \operatorname{supp}(\boldsymbol{v})$. From this fact, it follows that $\mathbf{P}_{t}^{n} h(x)=z^{n} h(x)$, for any $n \geq 1$ and $x \in \operatorname{supp}(\boldsymbol{v})$. This implies that $|h|(x) \leq \mathbf{P}^{n}|h|(x)$, for any $x \in \operatorname{supp}(\boldsymbol{v})$. Note also that $|h|$ belongs to $\mathscr{B}$. Therefore, as we have seen in the proof of the first claim, we have, $\lim _{n \rightarrow+\infty} \mathbf{P}^{n}|h|(x)=\boldsymbol{v}(|h|)=\mathbb{E}(|h|(Z))<+\infty$, for any $x \in \operatorname{supp}(\boldsymbol{v})$. So 
$|h|(x) \leq \int_{x^{\prime} \in \mathbb{R}^{d}}|h|\left(x^{\prime}\right) \boldsymbol{v}\left(\mathrm{d} x^{\prime}\right)$, for any $x \in \operatorname{supp}(\boldsymbol{v})$. Since $|h|$ is continuous, this implies that $|h|$ is constant on the support of $v$. In particular, this means that $h(x) \neq 0$ for any $x \in \operatorname{supp}(\boldsymbol{v})$.

Since the support of $\boldsymbol{v}$ is stable by all the elements of the support of $\boldsymbol{\mu}$, we deduce that the random variable $\xi_{n}(x)=\exp \left(i t\left\langle u, \sum_{k=1}^{n} g_{k} \cdots g_{1} \cdot x\right\rangle\right) h\left(g_{n} \cdots g_{1} \cdot x\right)$ takes values on the sphere $\mathbb{S}_{v(|h|)}=\{a \in \mathbb{C}:|a|=\boldsymbol{v}(|h|)\}$, for all $x$ in the support of $\boldsymbol{v}$. Moreover, the mean $z^{n} h(x)$ of $\xi_{n}(x)$ is also on $\mathbb{S}_{v(|h|)}$, which is possible only if $\xi_{n}(x)$ is a constant, for any $x \in \operatorname{supp}(\boldsymbol{v})$. Consequently, for any pair $x, y \in \operatorname{supp}(\boldsymbol{v})$, there exists an event $\Omega_{x, y}$ of $\mathbb{P}$-probability one such that on $\Omega_{x, y}$ it holds, for any $n \geq 1$,

$$
\exp \left(i t\left\langle u, \sum_{k=1}^{n} g_{k} \cdots g_{1} \cdot v\right\rangle\right) h\left(g_{n} \cdots g_{1} \cdot v\right)=z^{n} h(v),
$$

with $v \in\{x, y\}$, from which we get

$$
\frac{h\left(g_{n} \cdots g_{1} \cdot y\right)}{h\left(g_{n} \cdots g_{1} \cdot x\right)}=\frac{h(y)}{h(x)} \exp \left(i t\left\langle\sum_{k=1}^{n}{ }^{t} A_{1} \cdots{ }^{t} A_{k} u, x-y\right\rangle\right) .
$$

In addition, for any $n \geq 1$,

$$
\mathbb{E}\left(\left|\frac{h\left(g_{n} \cdots g_{1} \cdot y\right)}{h\left(g_{n} \cdots g_{1} \cdot x\right)}-1\right|\right)=\mathbb{E}\left(\left|\frac{h\left(g_{1} \cdots g_{n} \cdot y\right)}{h\left(g_{1} \cdots g_{n} \cdot x\right)}-1\right|\right) .
$$

Since, for $v \in\{x, y\}$, the sequence $h\left(g_{1} \cdots g_{n} \cdot v\right)$ converges a.s. to $h(Z)$ and since $h$ is bounded with a constant modulus, we have by (A.1),

$$
\begin{aligned}
0 & =\lim _{n \rightarrow+\infty} \mathbb{E}\left(\left|\frac{h\left(g_{n} \cdots g_{1} \cdot y\right)}{h\left(g_{n} \cdots g_{1} \cdot x\right)}-1\right|\right) \\
& =\lim _{n \rightarrow+\infty} \mathbb{E}\left(\left|\frac{h(y)}{h(x)} \exp \left(i t\left\langle\sum_{k=1}^{n}{ }^{t} A_{1} \cdot{ }^{t} A_{k} u, x-y\right\rangle\right)-1\right|\right) .
\end{aligned}
$$

Taking into account that the series $\sum_{k=1}^{n}{ }^{t} A_{1} \cdots{ }^{t} A_{k}$ converges a.s. to a random variable $Z^{\prime}$, we have for any $x, y \in \operatorname{supp}(\boldsymbol{v})$,

$$
\mathbb{E}\left(\left|\frac{h(y)}{h(x)} \mathrm{e}^{i t\left\langle Z^{\prime} u, x-y\right\rangle}-1\right|\right)=0 .
$$

Since the support of $\boldsymbol{v}$ is invariant by all the elements of the support of $\boldsymbol{\mu}$, by point 2 of Hypothesis 3.1, we deduce that the support of $\boldsymbol{v}$ is not contained in an affine subspace of $\mathbb{R}^{d}$, that is, for any $1 \leq j \leq d$, there exist $x_{j}, y_{j} \in \operatorname{supp}(\boldsymbol{v})$, such that the family $\left(v_{j}\right)_{1 \leq j \leq d}=\left(x_{j}-y_{j}\right)_{1 \leq j \leq d}$ generates $\mathbb{R}^{d}$. From (A.2), we conclude that for any $1 \leq j \leq d$,

$$
\frac{h\left(y_{j}\right)}{h\left(x_{j}\right)} \mathrm{e}^{i t\left\langle Z^{\prime} u, v_{j}\right\rangle}=1, \quad \mathbb{P} \text {-a.s. }
$$


Let $\theta_{j}$ be such that $\frac{h\left(x_{j}\right)}{h\left(y_{j}\right)}=\mathrm{e}^{i \theta_{j}}$. Denoting by $\boldsymbol{\eta}_{u}$ the distribution of $Z^{\prime} u$, we obtain that $\left\langle Z^{\prime} u, v_{j}\right\rangle \in \frac{\theta_{j}+2 \pi \mathbb{Z}}{t} \mathbb{P}$-a.s. and so the support of $\boldsymbol{\eta}_{u}$ is discrete. Moreover, the measure $\boldsymbol{\eta}_{u}$ is invariant for the Markov chain $X_{n+1}^{\prime}={ }^{t} A_{n+1}\left(X_{n}^{\prime}+u\right)$ and so, for any Borel set $B$ of $\mathbb{R}^{d}$,

$$
\boldsymbol{\eta}_{u}(B)=\mathbb{E}\left(\int_{v \in \mathbb{R}^{d}} \mathbb{1}_{B}\left({ }^{t} A_{1}(v+u)\right) \boldsymbol{\eta}_{u}(\mathrm{~d} v)\right) .
$$

Since $\boldsymbol{\eta}_{u}$ is discrete, the set $E_{\max }=\left\{x \in \mathbb{R}^{d}: \boldsymbol{\eta}_{u}(\{x\})=\max _{y \in \mathbb{R}^{d}} \boldsymbol{\eta}_{u}(\{y\})\right\}$ is nonempty and finite. Moreover, using (A.3) with $B=\{x\}$ and $x \in E_{\max }$, we can see that the image ${ }^{t} A_{1}^{-1} x-u$ belongs to $E_{\max } \mathbb{P}$-a.s. Denoting by $v_{0}$ the barycentre of $E_{\max }$, we find that

$$
\mathbb{P}\left({ }^{t} A_{1}^{-1} v_{0}-u=v_{0}\right)=1 .
$$

The fact that $u \neq 0$ implies that $v_{0} \neq 0$. The latter implies that ${ }^{t} A_{1}^{-1} v_{0}=v_{0}+u=$ ${ }^{t} A_{2}^{-1} v_{0}$ almost surely, which contradicts point 3 of Hypothesis 3.1 .

Conditions (b), (c) and (d) of the theorem of Ionescu Tulcea and Marinescu as stated in Chapter 3 of Norman [28] follow from points 1-3 of Lemma A.1, respectively. It remains to show condition (a). Let $\left(h_{n}\right)_{n \geq 0}$ be a sequence in $\mathscr{L}_{\theta, \varepsilon}$ satisfying $\left\|h_{n}\right\|_{\theta, \varepsilon} \leq K$, for any $n \geq 0$ and some constant $K$ and suppose that there exists $h \in \mathscr{C}\left(\mathbb{R}^{d}\right)$ such that $\lim _{n \rightarrow+\infty}\left|h_{n}-h\right|_{\theta}=0$. For any $x, y, z \in \mathbb{R}^{d}$ and $n \geq 0$,

$$
\begin{aligned}
& \frac{|h(x)-h(y)|}{|x-y|^{\varepsilon}(1+|x|)(1+|y|)}+\frac{|h(z)|}{(1+|z|)^{\theta}} \\
& \quad \leq\left|h_{n}-h\right|_{\theta}\left(\frac{(1+|x|)^{\theta}+(1+|y|)^{\theta}}{|x-y|^{\varepsilon}(1+|x|)(1+|y|)}+1\right)+\left[h_{n}\right]_{\varepsilon}+\left|h_{n}\right|_{\theta} .
\end{aligned}
$$

Taking the limit as $n \rightarrow+\infty$, shows that $h \in \mathscr{L}_{\theta, \varepsilon}$ and $\|h\|_{\theta, \varepsilon} \leq K$.

The theorem of Ionescu Tulcea and Marinescu and the unicity of the one-dimensional projector proved in point 1 of Lemma A.2 imply Hypothesis M2. Hypothesis M3 is obtained easily from Lemma A.1.

Point 2 of Lemma A. 2 will be used latter to prove that $\sigma^{2}>0$.

Proof of M4. By the hypothesis $\alpha=\frac{2+2 \delta}{1+\varepsilon}>2$. Consider the function $N: \mathbb{R}^{d} \rightarrow$ $\mathbb{R}_{+}$defined by $N(x)=|x|^{1+\varepsilon}$. For any $x, y \in \mathbb{R}^{d}$ satisfying $|x| \leq|y|$,

$$
|N(x)-N(y)| \leq(1+\varepsilon)|y|^{\varepsilon}|x-y| .
$$

Using the fact that $|N(x)-N(y)| \leq 2|y|^{1+\varepsilon}$, we have

$$
|N(x)-N(y)| \leq(1+\varepsilon)^{\varepsilon} 2^{1-\varepsilon}|y|^{\varepsilon^{2}+(1+\varepsilon)(1-\varepsilon)}|x-y|^{\varepsilon}=c_{\varepsilon}|y||x-y|^{\varepsilon} .
$$

Together with $|N|_{\theta}<+\infty$, this proves that the function $N$ is in $\mathscr{B}=\mathscr{L}_{\theta, \varepsilon}$. 
Obviously $|f(x)|^{1+\varepsilon}=|\langle u, x\rangle|^{1+\varepsilon} \leq|u|^{1+\varepsilon}(1+N(x))$. Moreover, for any $h \in$ $\mathscr{L}_{\theta, \varepsilon}$,

$$
|h(x)| \leq[h]_{\varepsilon}|x|^{\varepsilon}(1+|x|)+|h(0)| \leq 2\|h\|_{\theta, \varepsilon}(1+N(x))
$$

and so $\left\|\delta_{x}\right\|_{\mathscr{B}^{\prime}} \leq 2(1+N(x))$. Note that for any $p \in[1, \alpha]$,

$$
\begin{aligned}
\mathbb{E}^{1 / p} & \left(N\left(g_{n} \cdots g_{1} \cdot x\right)^{p}\right) \\
& \leq 2^{1+\varepsilon}\left(\mathbb{E}^{1 / p}\left(\left\|\Pi_{n}\right\|^{p(1+\varepsilon)}\right) N(x)+\mathbb{E}^{1 / p}\left(\left|g_{n} \cdots g_{1} \cdot 0\right|^{p(1+\varepsilon)}\right)\right) .
\end{aligned}
$$

Since $p(1+\varepsilon) \leq 2+2 \delta$, the previous inequality proves that $\mathbb{E}_{x}^{1 / p}\left(N\left(X_{n}\right)^{p}\right) \leq$ $c(1+N(x))$. Thus, we proved the first inequality of point 1 of M4.

For any $l \geq 1$, we consider the function $\phi_{l}$ on $\mathbb{R}_{+}$defined by

$$
\phi_{l}(t)= \begin{cases}0 & \text { if } t \leq l^{\frac{1}{1+\varepsilon}}-1, \\ t-\left(l^{\frac{1}{1+\varepsilon}}-1\right) & \text { if } t \in\left[l^{\frac{1}{1+\varepsilon}}-1, l^{\frac{1}{1+\varepsilon}}\right], \\ 1 & \text { if } t \geq l^{\frac{1}{1+\varepsilon}} .\end{cases}
$$

Define $N_{l}$ on $\mathbb{R}^{d}$ by $N_{l}(x)=\phi_{l}(|x|) N(x)$. For any $x \in \mathbb{R}^{d}$, we have $N(x) \mathbb{1}_{\{N(x)>l\}} \leq N_{l}(x) \leq N(x)$ which implies that $\left|N_{l}\right|_{\theta} \leq|N|_{\theta}<+\infty$. Moreover, for any $x, y \in \mathbb{R}^{d}$ satisfying $|x| \leq|y|$, we have

$$
\left|\phi_{l}(|y|)-\phi_{l}(|x|)\right| \leq \min (|y|-|x|, 1) .
$$

So

$$
\left|N_{l}(y)-N_{l}(x)\right| \leq[N]_{\varepsilon}|x-y|^{\varepsilon}(1+|x|)(1+|y|)+|x|^{1+\varepsilon}|y-x|^{\varepsilon} .
$$

Since $|x| \leq|y|$, we obtain that $\left[N_{l}\right]_{\varepsilon} \leq[N]_{\varepsilon}+1<+\infty$. Therefore, the function $N_{l}$ belongs to $\mathscr{B}=\mathscr{L}_{\theta, \varepsilon}$, which finishes the proof of point 1 of M4.

Moreover, $\left\|N_{l}\right\|_{\theta, \varepsilon} \leq\|N\|_{\theta, \varepsilon}+1$ and, so the point 2 of M4 is also established.

Since $\int_{\mathbb{X}}|x|^{p} \boldsymbol{v}(\mathrm{d} x)<+\infty$, for any $p \leq 2+2 \delta$, we find that

$$
\boldsymbol{v}\left(N_{l}\right) \leq \int_{\mathbb{X}}|x|^{1+\varepsilon} \mathbb{1}_{\left\{|x| \geq l \frac{1}{1+\varepsilon}-1\right\}} \boldsymbol{v}(\mathrm{d} x) \leq \frac{\int_{\mathbb{X}}|x|^{2+2 \delta} \boldsymbol{v}(\mathrm{d} x)}{\left(l \frac{1}{1+\varepsilon}-1\right)^{2+2 \delta-(1+\varepsilon)}} .
$$

Choosing $\beta=\alpha-2>0$, we obtain point 3 of M4.

Proof of M5. Using (2.5) and the point 4 of Hypothesis 3.1,

$$
\mu=\int_{\mathbb{R}^{d}}\langle u, x\rangle \boldsymbol{v}(\mathrm{d} x)=\left\langle u, \mathbb{E}\left(\sum_{k=1}^{+\infty} A_{1} \cdots A_{k-1} B_{k}\right)\right\rangle=0 .
$$

Now we prove that $\sigma^{2}>0$. For this, suppose the contrary: $\sigma^{2}=0$. One can easily check that the function $f$ belongs to $\mathscr{B}$. Using M2 and the fact that $\nu(f)=\mu=0$, we deduce that $\sum_{n \geq 0}\left\|\mathbf{P}^{n} f\right\|_{\theta, \varepsilon}=\sum_{n \geq 0}\left\|Q^{n} f\right\|_{\theta, \varepsilon}<+\infty$ and therefore the series 
$\sum_{n \geq 0} \mathbf{P}^{n} f$ converges in $\left(\mathscr{B},\|\cdot\|_{\theta, \varepsilon}\right)$. We denote by $\Theta \in \mathscr{B}$ its limit and notice that the function $\Theta$ satisfies the Poisson equation: $\Theta-\mathbf{P} \Theta=f$.

Using the bound (2.6), we have $\left|\sum_{n=1}^{N} f(x) \mathbf{P}^{n} f(x)\right| \leq c(1+N(x))^{2}$. By the Lebesgue dominated convergence theorem, from (2.5), we obtain

$$
\begin{aligned}
\sigma^{2} & =\int_{\mathbb{R}^{d}} f(x)(2 \Theta(x)-f(x)) \boldsymbol{v}(\mathrm{d} x) \\
& =\int_{\mathbb{R}^{d}}\left(\Theta^{2}(x)-(\mathbf{P} \Theta)^{2}(x)\right) \boldsymbol{v}(\mathrm{d} x) \\
& =\int_{\mathrm{GL}(d, \mathbb{R}) \times \mathbb{R}^{d} \times \mathbb{R}^{d}}\left(\Theta\left(g_{1} \cdot x\right)-\mathbf{P} \Theta(x)\right)^{2} \boldsymbol{\mu}\left(\mathrm{d} g_{1}\right) \boldsymbol{v}(\mathrm{d} x) .
\end{aligned}
$$

As $\sigma^{2}=0$, we have $\Theta\left(g_{1} \cdot x\right)=\mathbf{P} \Theta(x)$, that is, $f\left(g_{1} \cdot x\right)=\mathbf{P} \Theta(x)-\mathbf{P} \Theta\left(g_{1} \cdot x\right)$, $\boldsymbol{\mu} \times \boldsymbol{v}$-a.s. Consequently, there exists a Borel subset $B_{0}$ of $\mathbb{R}^{d}$ such that $\boldsymbol{v}\left(B_{0}\right)=1$ and for any $t \in \mathbb{R}$ and $x \in B_{0}$,

$$
\int_{\mathrm{GL}(d, \mathbb{R}) \times \mathbb{R}^{d}} \mathrm{e}^{i t\left\langle u, g_{1} \cdot x\right\rangle} \mathrm{e}^{i t \mathbf{P} \Theta\left(g_{1} \cdot x\right)} \boldsymbol{\mu}\left(\mathrm{d} g_{1}\right)=\mathrm{e}^{i t \mathbf{P} \Theta(x)} .
$$

Since the functions in the both sides are continuous, this equality holds for every $x \in \operatorname{supp}(\boldsymbol{v})$. Since $\Theta \in \mathscr{L}_{\theta, \varepsilon}$, the function $x \mapsto \mathrm{e}^{i t \mathbf{P} \Theta(x)}$ belongs to $\mathscr{L}_{\theta, \varepsilon} \backslash\{0\}$. This contradicts the point 2 of Lemma A.2 and we conclude that $\sigma^{2}>0$ and so M5 holds true.

\section{APPENDIX B: PROOFS FOR COMPACT MARKOV CHAINS}

In this section, we prove Proposition 3.7. For this, we show that M1-M5 hold true with $N=N_{l}=0$, for the Markov chain $\left(X_{n}\right)_{n \geq 1}$, the function $f$ and the Banach space $\mathscr{L}(\mathbb{X})$ given in Section 3.2.

Proof of M1. Obviously the Dirac measure belongs to $\mathscr{L}(\mathbb{X})^{\prime}$ and $\left\|\boldsymbol{\delta}_{x}\right\|_{\mathscr{L}(\mathbb{X})^{\prime} \leq}$ 1 for any $x \in \mathbb{X}$. For any $h \in \mathscr{L}(\mathbb{X})$ and $t \in \mathbb{R}$ the function $\mathrm{e}^{i t f} h$ belongs to $\mathscr{L}(\mathbb{X})$ and

$$
\left\|\mathrm{e}^{i t f} h\right\|_{\mathscr{L}} \leq|t|[f]_{\mathbb{X}}\|h\|_{\infty}+\|h\|_{\mathscr{L}} \leq\left(|t|[f]_{\mathbb{X}}+1\right)\|h\|_{\mathscr{L}} .
$$

Proof of M2. Let $\left(x_{1}, x_{2}\right)$ and $\left(y_{1}, y_{2}\right)$ be two elements of $\mathbb{X}$ and $h \in \mathscr{L}(\mathbb{X})$. Since

$$
\mathbf{P} h\left(x_{1}, x_{2}\right)=\int_{X} h\left(x_{2}, x^{\prime}\right) P\left(x_{2}, \mathrm{~d} x^{\prime}\right),
$$

we have $\|\mathbf{P} h\|_{\infty} \leq\|h\|_{\infty}$. Denote by $h_{x_{2}}$ the function $z \mapsto h\left(x_{2}, z\right)$, which is an element of $\mathscr{L}(X)$. Since $\left[h_{x_{2}}\right]_{X} \leq[h]_{\mathbb{X}}$ and $\left|h_{x_{2}}\right|_{\infty} \leq\|h\|_{\infty}$, we obtain also that

$$
\begin{aligned}
\left|\mathbf{P} h\left(x_{1}, x_{2}\right)-\mathbf{P} h\left(y_{1}, y_{2}\right)\right| & =\left|P h_{x_{2}}\left(x_{2}\right)-P h_{y_{2}}\left(y_{2}\right)\right| \\
& \leq\left[P h_{x_{2}}\right]_{X} d_{X}\left(x_{2}, y_{2}\right)+[h]_{\mathbb{X}} d_{X}\left(x_{2}, y_{2}\right) \\
& \leq\left(|P| \mathscr{L} \rightarrow \mathscr{L}\|h\|_{\mathbb{X}}+[h]_{\mathbb{X}}\right) d_{X}\left(x_{2}, y_{2}\right),
\end{aligned}
$$


where $|P|_{\mathscr{L} \rightarrow \mathscr{L}}$ is the norm of the operator $P: \mathscr{L}(X) \rightarrow \mathscr{L}(X)$. Therefore, $\mathbf{P}$ is a bounded operator on $\mathscr{L}(\mathbb{X})$ and $\|\mathbf{P}\|_{\mathscr{L} \rightarrow \mathscr{L}} \leq\left(1+|P|_{\mathscr{L} \rightarrow \mathscr{L}}\right)$. Now, for any $h \in \mathscr{L}(\mathbb{X})$, we define the function $F_{h}$ by

$$
F_{h}\left(x_{2}\right):=\int_{X} h\left(x_{2}, x^{\prime}\right) P\left(x_{2}, \mathrm{~d} x^{\prime}\right)=\mathbf{P} h\left(x_{1}, x_{2}\right) .
$$

Notice that $F_{h}$ belongs to $\mathscr{L}(X)$ and $\left|F_{h}\right|_{\mathscr{L}} \leq\|\mathbf{P} h\|_{\mathscr{L}}$. So by Proposition 3.5, for any $n \geq 2,\left(x_{1}, x_{2}\right) \in \mathbb{X}$ and $h \in \mathscr{L}(\mathbb{X})$,

$$
\begin{aligned}
\mathbf{P}^{n} h\left(x_{1}, x_{2}\right) & =P^{n-1} F_{h}\left(x_{2}\right)=v\left(F_{h}\right)+R^{n-1} F_{h}\left(x_{2}\right) \\
& =\boldsymbol{v}(h) e\left(x_{1}, x_{2}\right)+Q^{n} h\left(x_{1}, x_{2}\right),
\end{aligned}
$$

where the probability $\boldsymbol{v}$ is defined on $\mathbb{X}$ by

$$
\boldsymbol{v}(h)=v\left(F_{h}\right)=\int_{X \times X} h\left(x^{\prime}, x^{\prime \prime}\right) P\left(x^{\prime}, \mathrm{d} x^{\prime \prime}\right) v\left(\mathrm{~d} x^{\prime}\right),
$$

the function $e$ is the unit function on $\mathbb{X}, e\left(x_{1}, x_{2}\right)=1, \forall\left(x_{1}, x_{2}\right) \in \mathbb{X}$ and $Q$ is the linear operator on $\mathscr{L}(\mathbb{X})$ defined by $Q h=R\left(F_{h}\right)=\mathbf{P} h-\boldsymbol{v}(h)$. By Proposition 3.5, the operator $Q$ is bounded and for any $n \geq 1,\left\|Q^{n}\right\|_{\mathscr{L} \rightarrow \mathscr{L}} \leq$ $\left|R^{n-1}\right| \mathscr{L} \rightarrow \mathscr{L}\|\mathbf{P}\|_{\mathscr{L} \rightarrow \mathscr{L}} \leq c \mathrm{e}^{-c n}$. Since $v$ is invariant by $P$, one can easily verify that $\Pi Q=Q \Pi=0$, where $\Pi$ is the one-dimensional projector defined on $\mathscr{L}(\mathbb{X})$ by $\Pi h=v(h) e$.

Proof of M3. For any $t \in \mathbb{R}, h \in \mathscr{L}(\mathbb{X})$ and $\left(x_{1}, x_{2}\right) \in \mathbb{X}$,

$$
\mathbf{P}_{t} h\left(x_{1}, x_{2}\right)=\int_{X} \mathrm{e}^{i t f\left(x_{2}, x^{\prime}\right)} h\left(x_{2}, x^{\prime}\right) P\left(x_{2}, \mathrm{~d} x^{\prime}\right)=\sum_{n=0}^{+\infty} \frac{i^{n} t^{n}}{n !} L_{n}(h)\left(x_{1}, x_{2}\right),
$$

where $L_{n}(h)=\mathbf{P}\left(f^{n} h\right)$. Since $\left(\mathscr{L}(\mathbb{X}),\|\cdot\|_{\mathscr{L}}\right)$ is a Banach algebra, it follows that $L_{n}$ is a bounded operator on $\mathscr{L}(\mathbb{X})$ and $\left\|L_{n}\right\|_{\mathscr{L} \rightarrow \mathscr{L}} \leq\|\mathbf{P}\|_{\mathscr{L} \rightarrow \mathscr{L}}\|f\|_{\mathscr{L}}^{n}$. Consequently, the application $t \mapsto \mathbf{P}_{t}$ is analytic on $\mathbb{R}$ and so, by the analytic perturbation theory of linear operators (see [26]), there exists $\varepsilon_{0}>0$ such that, for any $|t| \leq \kappa$,

$$
\mathbf{P}_{t}^{n}=\lambda_{t}^{n} \Pi_{t}+Q_{t}^{n},
$$

where $\lambda_{t}$ is an eigenvalue of $\mathbf{P}_{t}, \Pi_{t}$ is the projector on the one-dimensional eigenspace of $\lambda_{t}$ and $Q_{t}$ is an operator of spectral radius $r\left(Q_{t}\right)<\left|\lambda_{t}\right|$ such that $\Pi_{t} Q_{t}=Q_{t} \Pi_{t}=0$. The functions $t \mapsto \lambda_{t}, t \mapsto \Pi_{t}$ and $t \mapsto Q_{t}$ are analytic on $[-\kappa, \kappa]$. Furthermore, for any $h \in \mathscr{L}(\mathbb{X})$ and $\left(x_{1}, x_{2}\right) \in \mathbb{X}$,

$$
\left|\mathbf{P}_{t} h\right|\left(x_{1}, x_{2}\right)=\left|\int_{X} \mathrm{e}^{i t f\left(x_{2}, x^{\prime}\right)} h\left(x_{2}, x^{\prime}\right) P\left(x_{2}, \mathrm{~d} x^{\prime}\right)\right| \leq\|h\|_{\infty}
$$

and necessarily $\left|\lambda_{t}\right| \leq 1$, for any $|t| \leq \kappa$. Consequently,

$$
\sup _{|t| \leq \kappa, n \geq 1}\left\|\mathbf{P}_{t}^{n}\right\|_{\mathscr{L} \rightarrow \mathscr{L}} \leq c .
$$

Proof of M4 and M5. Since for any $x \in \mathbb{X},|f(x)| \leq|f|_{\infty}$ and $\left\|\boldsymbol{\delta}_{x}\right\|_{\mathscr{L}(\mathbb{X})^{\prime}} \leq 1$, we can choose $N=0$ and $N_{l}=0$ for any $l \geq 1$ and Hypothesis M4 is obviously satisfied.

Finally, Hypothesis 3.6 ensures that M5 holds true. 
Acknowledgements. The authors are very grateful to an anonymous referee for valuable comments and corrections which helped them improve the first version of this work.

\section{REFERENCES}

[1] Bertoin, J. and Doney, R. A. (1994). On conditioning a random walk to stay nonnegative. Ann. Probab. 22 2152-2167. MR1331218

[2] Bolthausen, E. (1976). On a functional central limit theorem for random walks conditioned to stay positive. Ann. Probab. 4 480-485. MR0415702

[3] Borovkov, A. A. (2004). On the asymptotic behavior of distributions of first-passage times, I. Math. Notes 75 23-37. MR2053147

[4] Borovkov, A. A. (2004). On the asymptotics of distributions of first-passage times, II. Math. Notes 75 322-330. MR2068798

[5] Buraczews Ki, D., Damek, E. and GuivarC'H, Y. (2010). Convergence to stable laws for a class of multidimensional stochastic recursions. Probab. Theory Related Fields 148333 402. MR2678893

[6] Caravenna, F. (2005). A local limit theorem for random walks conditioned to stay positive. Probab. Theory Related Fields 133 508-530. MR2197112

[7] Dembo, A., Ding, J. and Gao, F. (2013). Persistence of iterated partial sums. Ann. Inst. Henri Poincaré Probab. Stat. 49 873-884. MR3112437

[8] Denisov, D., Korshunov, D. and Wachtel, V. (2013). Harmonic functions and stationary distributions for asymptotically homogeneous transition kernels on $\digamma^{+}$. Preprint. Available at arXiv:1312.2201[math].

[9] Denisov, D. and WAChtel, V. (2010). Conditional limit theorems for ordered random walks. Electron. J. Probab. 15 292-322. MR2609589

[10] Denisov, D. and Wachtel, V. (2015). Exit times for integrated random walks. Ann. Inst. Henri Poincaré Probab. Stat. 51 167-193. MR3300967

[11] Denisov, D. and Wachtel, V. (2015). Random walks in cones. Ann. Probab. 43 992-1044. MR3342657

[12] DoneY, R. A. (1989). On the asymptotic behaviour of first passage times for transient random walk. Probab. Theory Related Fields 81 239-246. MR0982656

[13] DuRAJ, J. (2014). Random walks in cones: The case of nonzero drift. Stochastic Process. Appl. 124 1503-1518. MR3163211

[14] Eichelsbacher, P. and KöNIG, W. (2008). Ordered random walks. Electron. J. Probab. 13 1307-1336. MR2430709

[15] Feller, W. (1971). An Introduction to Probability Theory and Its Applications, Vol. II, 2nd ed. Wiley, New York. MR0270403

[16] GaO, Z., Guivarc'H, Y. and Le PAGe, É. (2015). Stable laws and spectral gap properties for affine random walks. Ann. Inst. Henri Poincaré Probab. Stat. 51 319-348. MR3300973

[17] Gordin, M. I. (1969). The central limit theorem for stationary processes. Dokl. Akad. Nauk SSSR 188 739-741. MR0251785

[18] Grama, I., Lauvergnat, R. and Le Page, É. (2018). Limit theorems for affine Markov walks conditioned to stay positive. Ann. Inst. Henri Poincaré Probab. Stat. 54 529-568. MR3765899

[19] Grama, I., Le Page, É. and Peigné, M. (2014). On the rate of convergence in the weak invariance principle for dependent random variables with applications to Markov chains. Colloq. Math. 134 1-55. MR3164936

[20] Grama, I., Le Page, É. and Peigné, M. (2017). Conditioned limit theorems for products of random matrices. Probab. Theory Related Fields 168 601-639. MR3663626 
[21] GuivarC'H, Y. and Hardy, J. (1988). Théorèmes limites pour une classe de chaînes de Markov et applications aux difféomorphismes d'Anosov. Ann. Inst. Henri Poincaré B, Probab. Stat. 24 73-98. MR0937957

[22] Guivarc'H, Y. and Le Page, E. (2008). On spectral properties of a family of transfer operators and convergence to stable laws for affine random walks. Ergodic Theory Dynam. Systems 28 423-446. MR2408386

[23] IGLeHART, D. L. (1974). Functional central limit theorems for random walks conditioned to stay positive. Ann. Probab. 2 608-619. MR0362499

[24] IgleharT, D. L. (1974). Random walks with negative drift conditioned to stay positive. J. Appl. Probab. 11 742-751. MR0368168

[25] Ionescu Tulcea, C. T. and Marinescu, G. (1950). Théorie ergodique pour des classes d'opérations non complètement continues. Ann. of Math. (2) 52 140-147. MR0037469

[26] Kato, T. (1976). Perturbation Theory for Linear Operators. 2nd ed. Springer, Berlin. MR0407617

[27] LÉVy, P. (1937). Théorie de L'addition des Variables Aléatoires. Gauthier-Villars, Paris.

[28] Norman, M. F. (1972). Markov Processes and Learning Models. Academic Press, New York. MR0423546

[29] Presman, È. L. (1967). A boundary value problem for the sum of lattice random variables given on a finite regular Markov chain. Teor. Verojatnost. i Primenen. 12 373-380. MR0214142

[30] Presman, È. L. (1969). Factorization methods, and a boundary value problem for sums of random variables given on a Markov chain. Izv. Ross. Akad. Nauk Ser. Mat. 33 861-900. MR0256467

[31] SpitZer, F. (1976). Principles of Random Walk. 2nd ed. Springer, New York. MR0388547

[32] Varopoulos, N. Th. (1999). Potential theory in conical domains. Math. Proc. Cambridge Philos. Soc. 125 335-384. MR1643806

[33] Varopoulos, N. Th. (2000). Potential theory in conical domains. II. Math. Proc. Cambridge Philos. Soc. 129 301-319. MR1765917

[34] Varopoulos, N. Th. (2001). Potential theory in Lipschitz domains. Canad. J. Math. 53 1057-1120. MR1859766

[35] VAtutin, V. A. and WACHTEL, V. (2009). Local probabilities for random walks conditioned to stay positive. Probab. Theory Related Fields 143 177-217. MR2449127

\author{
UNIVERSITÉ DE BRETAGNE SUD \\ LMBA UMR CNRS 6205 \\ VANNES \\ FRANCE \\ E-MAIL: ion.grama@univ-ubs.fr \\ ronan.lauvergnat@univ-ubs.fr \\ emile.le-page@univ-ubs.fr
}

